GRADUATE SCHOOL

\title{
INVESTIGATION OF THE BEHAVIOR OF VENTILATED SUPERCAVITIES
}

\section{A THESIS \\ SUBMITTED TO THE FACULTY OF THE GRADUATE SCHOOL OF THE UNIVERSITY OF MINNESOTA \\ BY}

ELLISON GORDON KAWAKAMI

IN PARTIAL FULFILLMENT OF THE REQUIREMENTS

FOR THE DEGREE OF

MASTER OF SCIENCE

ADVISOR: Dr. ROGER ARNDT

JANUARY 2011 
(C) Ellison Gordon Kawakami 2010 


\section{ACKNOWLEDGMENTS}

I would like to thank my advisor, Roger Arndt, for his continued guidance, wisdom, and patience throughout all of my graduate studies at the University of Minnesota. It has been a great honor to work for one of the most well-renowned researchers in the world in the area of cavitation. Without him, my thesis and graduate work would not have been possible. I also need to thank Martin Wosnik and William Hambleton for introducing me to experimental fluid mechanics, which has become my area of greatest interest. Martin's attention to detail combined with Will's knack for adapting to the ever constant, unforeseen challenges of experimentation made me into the researcher I am today. Their guidance provided the groundwork for the skills necessary to conduct my research. I am also very grateful for various researchers and other students that I have worked with during my time at Saint Anthony for their constant support. It has been an experience that I will never forget.

A special thanks to Mario Costello and Dave Hultman from the Electrical Engineering shop on campus for their work in making all of the models and components necessary for my research. It would have been a nightmare otherwise. Also many thanks to Gary Balas and Arnar Hjartarson from the Aerospace Department for providing me with insight as to how my research can be directly applied. It helped significantly to see the larger picture.

Funding for this project was provided by the Office of Naval Research. Dr. Kam Ng and Dr. Ronald Joslin were the grant monitors. Without their funding, this research would not have been possible.

Lastly, I need to thank my other friends and family. To all my friends for reminding me to keep things in perspective and for all the memories we shared during my time in graduate school. To my parents for their love and the sacrifices that they made to make my college education possible. I cannot thank them enough for what they have done for me and for making me into the person I am today. To my older brother, Damien, for paving the way for me.

Ad Majorem Dei Gloriam 


\begin{abstract}
A study was carried out to investigate various aspects of an axisymmetric, artificially supercavitating vehicle. One of the main focuses of the study was to investigate the effects of blockage introduced when conducting experiments in a water tunnel. Blockage influenced all of the experiments investigated. The effect of blockage was to set a lower limit on the cavitation number attainable. Experimental results were compared to numerical results from previous researchers. Froude number effects on cavitation number and supercavity dimensions were investigated. An advantage of this study over previous supercavitation experiments conducted at Saint Anthony Falls Laboratory is the ability to directly measure the pressure inside the supercavity. This knowledge is important for an underwater vehicle that requires drag reduction, by means of an artificial supercavity, to accelerate to speeds where natural supercavitation is possible. Next, the effects of model configuration are investigated in two ways. The first is the effect of the size of disc cavitator, and the second is the influence of the presence of a body inside the supercavity. For a supercavity, the method of closure and air entrainment is an important design consideration. For this study, a detailed examination of the closure method for various model configurations was explored. Finally, an effort was made to correlate water tunnel experiments with unbounded flow. This was done by computing an equivalent unbounded flow cavitation number from values obtained in a water tunnel.
\end{abstract}


TABLE OF CONTENTS

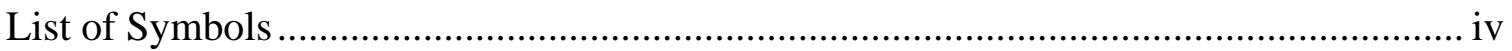

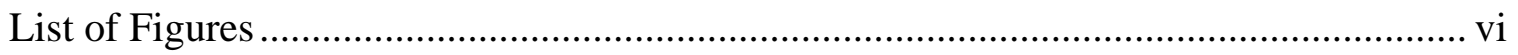

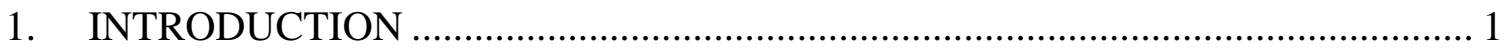

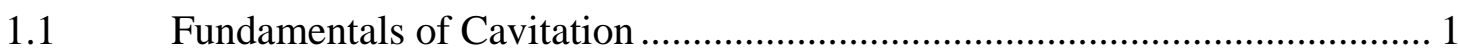

1.2 Introduction to Supercavitation................................................................. 3

1.2.1 Natural vs. Artificial Supercavitation .......................................................... 4

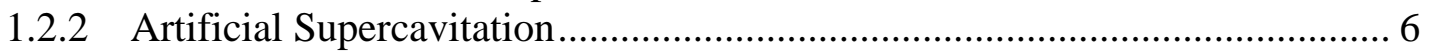

$1.3 \quad$ Supercavitation Advantages.................................................................... 9

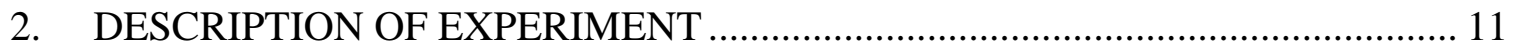

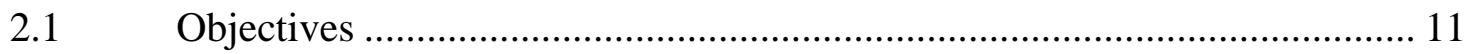

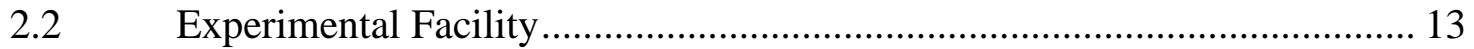

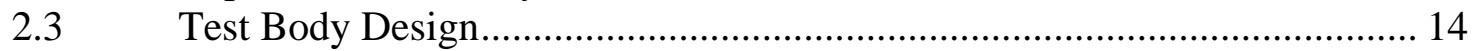

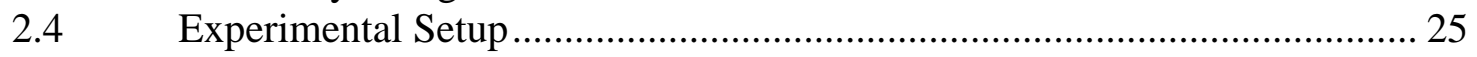

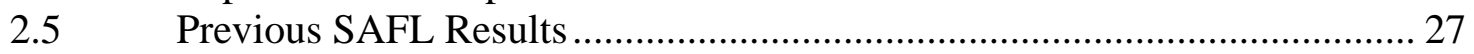

3. DISCUSSION AND EXPERIMENTAL RESULTS ............................................... 30

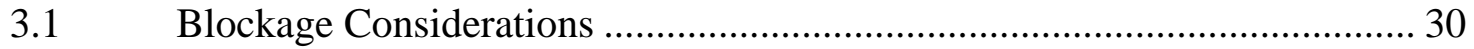

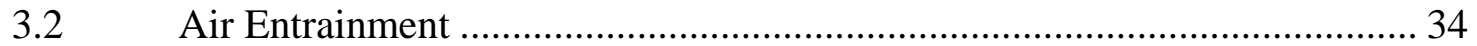

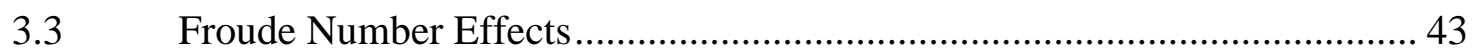

$3.4 \quad$ Unbounded Flow Equivalence................................................................... 50

3.5 Cavity Closure Mechanisms ……………............................................ 54

3.6 Model Comparison............................................................................... 56

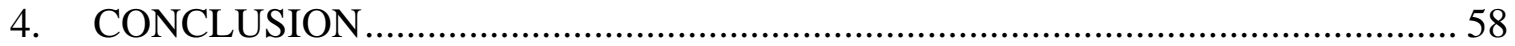

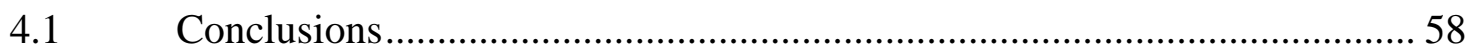

4.2 Recommendations for Future Work.......................................................... 60

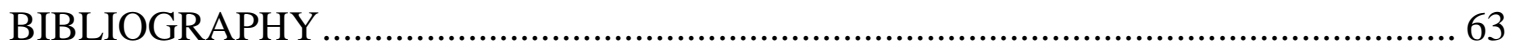

APPENDIX A: Pressure Transducer Calibration Procedure ............................................... 66

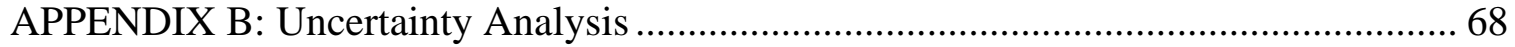

APPENDIX C: Matlab code for supercavity analysis ...................................................... 70 


\section{List of Symbols}

$\mathrm{a}^{*} \quad$ critical cavitator angle of attack for quad-vortex closure method

A intersectional area of supercavity

$\mathrm{A}_{\mathrm{c}} \quad$ cross sectional area of supercavity its maximum diameter

$\mathrm{A}_{0} \quad$ cross sectional area of the SAFL water tunnel test section

$\mathrm{C}_{\mathrm{xo}}$ drag coefficient at a cavitation number of zero

$\mathrm{C}_{\mathrm{p}, \min } \quad$ minimum pressure coefficient

$\mathrm{C}_{\mathrm{D}} \quad$ drag coefficient

$\mathrm{C}_{\mathrm{q}} \quad$ air entrainment coefficient

$\mathrm{d}_{\mathrm{c}} \quad$ cavitator diameter

D SAFL water tunnel test section diameter

$\mathrm{D}_{\mathrm{c}} \quad$ maximum supercavity diameter

$\mathrm{D} / \mathrm{d}_{\mathrm{c}} \quad$ blockage ratio

Fr Froude number

g gravitational acceleration

$1 \quad$ characteristic length

L twice the cavity half length, the distance from the cavitator to the point of maximum diameter.

$\mathrm{P}_{\mathrm{C}} \quad$ cavity pressure

$\mathrm{P}_{\mathrm{m}} \quad$ minimum pressure

$\mathrm{P}_{\mathrm{o}} \quad$ stagnation pressure in the settling chamber upstream of the SAFL test section

$\mathrm{P}_{\mathrm{v}} \quad$ vapor pressure of liquid

$\mathrm{P}_{2} \quad$ pressure after the pressure drop associated with hydrofoil drag

$\mathrm{P}_{\infty} \quad$ freestream static pressure

$\dot{Q} \quad$ volumetric flow rate

$\mathrm{R}_{\mathrm{k}} \quad$ universal gas constant

S hydrofoil wing area

$\mathrm{U}_{\mathrm{c}} \quad$ velocity along the surface of the supercavity

$\mathrm{U}_{\infty} \quad$ freestream velocity

$\mathrm{U}_{ \pm} \quad$ velocity along the top (+) or bottom (-) surface of the supercavity 
$\begin{array}{ll}\mathrm{z}_{ \pm} & \text {height above }(+) \text { or below (-) the centerline to the surface of the supercavity } \\ \beta & \text { ratio between vaporous and artificial cavitation numbers } \\ \Gamma & \begin{array}{l}\text { circulation due to the difference in velocity between the top and bottom surfaces } \\ \text { of the supercavity }\end{array} \\ \rho & \text { fluid density } \\ \sigma & \text { cavitation number } \\ \sigma_{\mathrm{C}} & \quad \text { cavitation number based on cavity pressure } \\ \sigma_{\mathrm{i}} & \text { incipient cavitation number } \\ \sigma_{\mathrm{min}} & \text { minimum cavitation number } \\ \sigma_{\mathrm{v}} & \text { vaporous cavitation number } \\ \sigma_{\infty} & \text { freestream cavitation number }\end{array}$ 


\section{List of Figures}

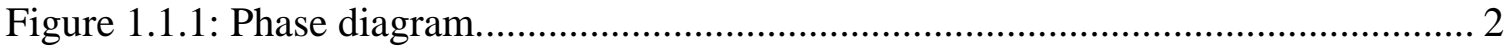

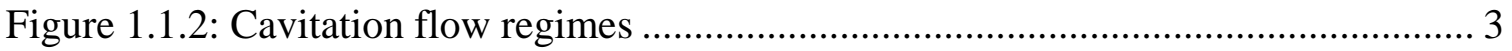

Figure 1.2.1.1: Three phases of developed cavitation ............................................... 6

Figure 1.2.2.1: Schematic of re-entrant jet regime .................................................... 7

Figure 1.2.2.2: Schematic of twin-vortex mechinism of supercavity closure .................... 8

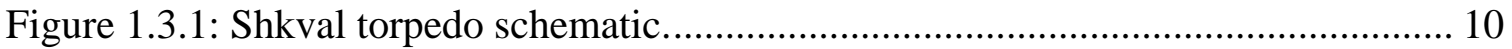

Figure 2.2.1: Schematic of SAFL high speed water tunnel. ........................................ 13

Figure 2.3.1: Forward Facing Model ....................................................................... 15

Figure 2.3.2: Forward facing model nozzle and cavitator assembly. ............................ 16

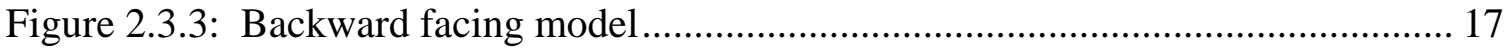

Figure 2.3.4: Backward facing model 10mm cavitator, original design......................... 17

Figure 2.3.5: Backward facing model 20mm cavitator, modified design........................ 18

Figure 2.3.6: Supercavity development for the jet-type cavitator ................................. 20

Figure 2.3.7: Supercavity development for the second generation of cavitator .............. 21

Figure 2.3.8: Comparison of supercavities created by the two models ........................... 22

Figure 2.3.9: Problem description for choking phenomenon. ....................................... 23

Figure 2.4.1: Experimental setup schematic .......................................................... 26

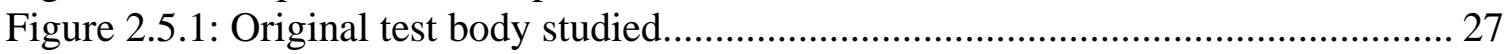

Figure 2.5.2: Air entrainment data for both disks and struts ...................................... 28

Figure 2.5.3: Typical entrainment plot as observed by Xu.......................................... 29

Figure 3.1.1: Tulin (1961) predictions for minimum cavitation number......................... 31

Table 3.1.1: Summary of blockage ratio, $\mathrm{D} / \mathrm{d}_{\mathrm{c}}$, values and corresponding minimum

cavitation values as predicted by Brennen (1969). .................................................... 32

Figure 3.1.2: Experimental data compared to Brennen’s numerical predictions.............. 33

Figure 3.2.1: Typical entrainment curve (Swanson and O’Neill, 1951)......................... 34

Figure 3.2.2: Modified form of entrainment coefficient plotted against cavitation number

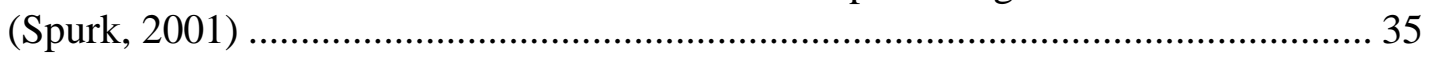

Figure 3.2.3: Backward facing model cavity development ......................................... 36

Figure 3.2.4: Forward facing model cavity development ............................................. 36

Figure 3.2.5: Ratio of cavitation numbers, $\sigma_{\mathrm{c}} / \sigma_{\min }$, plotted against Froude number ........ 39

Figure 3.2.6: Comparison of the two types of re-entrant jet closure methods.................. 41

Figure 3.2.7: Supercavity development. Backward facing model ................................ 42

Figure 3.3.1: Ventilation curves for supercavities due to small disks ........................... 43

Figure 3.3.2: Entrainment Coefficient plotted against cavitation number for constant

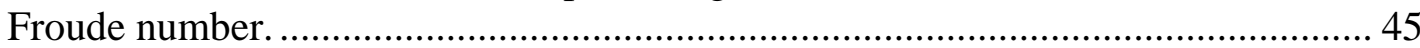

Figure 3.3.3: Cavitation number plotted against Froude number for constant entraintment

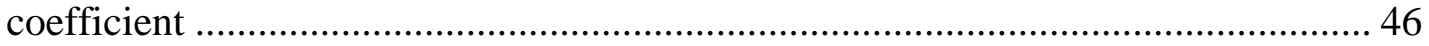

Figure 3.3.4: Comparison of cavitation numbers at constant entrainment coefficient, $\mathrm{C}_{\mathrm{q}}=$ constant, for the 10 and 20mm disk cavitators with the backward and forward facing models.

Figure 3.3.5: Ventilated cavitation number plotted against Froude number for a $10 \mathrm{~mm}$ cavitator with the forward facing model

Figure 3.3.6: Ventilated cavitation number plotted against Froude number for a $10 \mathrm{~mm}$ cavitator with the backward facing model 
Figure 3.4.1: Comparison of experimental data to Garabedian's predictions for

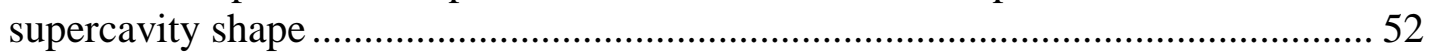

Figure 3.4.2: Freestream cavitation number plotted against Froude number .................... 53

Figure 3.5.1: Examples of twin-vortex closure for both models ....................................... 55

Figure 3.5.2: Example of quad-vortex closure................................................................. 56

Figure 3.6.1: Control volume for analysis of hydrofoil drag............................................ 57 


\section{INTRODUCTION}

Cavitation occurs in a variety of hydrodynamic applications. These applications include, but are not limited to, hydraulic pumps and motors, hydrofoils, propellers and spillways. For many applications, cavitation has negative effects. For many manufacturers, great efforts are made to reduce or eliminate cavitation. Cavitation can alter the performance of a hydraulic system (reduction in lift and increase in drag for a hydrofoil, decrease in turbomachinery efficiency, reduced capacity to evacuate water in spillways, unwanted noise, etc.). While the effects of cavitation are generally negative, it does have advantageous effects in certain applications, such as the homogenization of milk, cleaning of surfaces by cavitating jets and drag reduction by means of supercavitation. Depending on the application, cavitation provides different issues that the designer must address.

The application of drag reduction by means of supercavitation has been an area of great interest. Although the concept itself is simple enough, its application is challenging due the highly complex physics involved.

\subsection{Fundamentals of Cavitation}

Cavitation can be defined as the breakdown of a liquid medium under very low pressures. Visually, cavitation can be observed in the formation of vaporous bubbles, or cavities in a liquid. In this sense, cavitation is very similar to the boiling of water. For boiling, the breakdown of the liquid medium occurs as a result of an increase in temperature at nearly constant pressure. The difference with cavitation is that the breakdown of the liquid medium results from a decrease in pressure at nearly constant temperature. This is illustrated in Figure 1.1.1. 


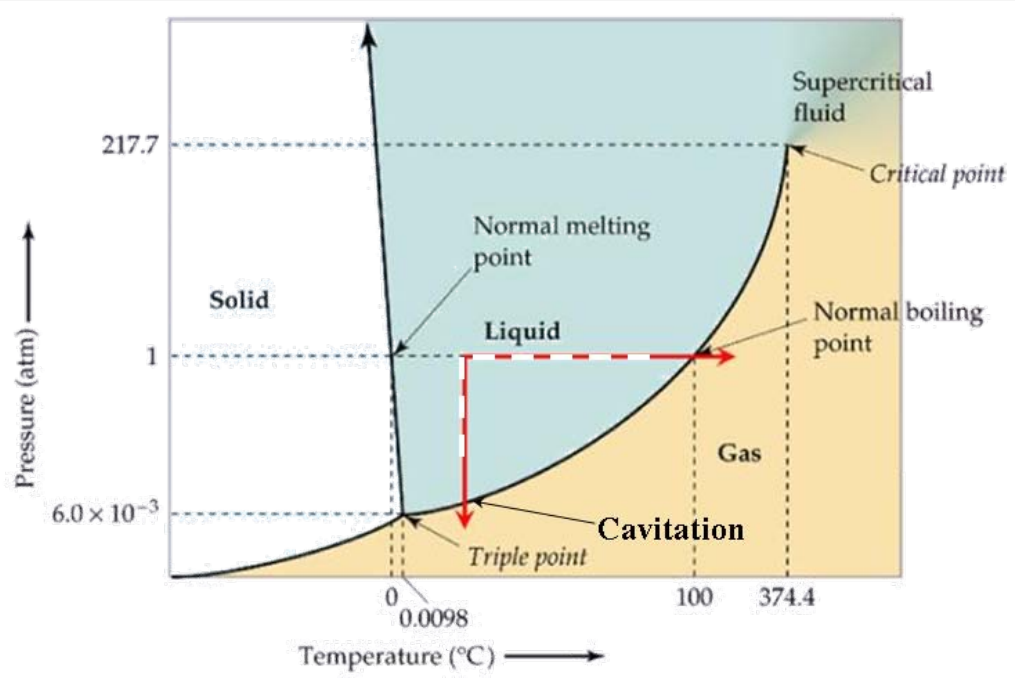

Figure 1.1.1: Phase diagram.

The fundamental parameter used to describe cavitation is the cavitation number

$$
\sigma_{v}=\frac{P_{\infty}-P_{v}}{\frac{1}{2} \rho U_{\infty}^{2}}
$$

where $P_{\infty}$ is the static freestream pressure, $P_{v}$ is the vapor pressure of water, $\rho$ is the density of the liquid, and $U_{\infty}$ is the freestream velocity. The cavitation number corresponding to the initial formation of vaporous bubbles (inception) in an initially single phase flow is defined as

$$
\sigma_{i}=-C_{P, \text { min }}
$$

where $\mathrm{Cp}_{\min }$ is the minimum pressure coefficient, which is normally negative. The minimum pressure coefficient is expressed as

$$
C_{p, \min }=\frac{P_{m}-P_{\infty}}{\frac{1}{2} \rho U_{\infty}^{2}}
$$

where $\mathrm{P}_{\mathrm{m}}$ is the minimum pressure. For a liquid it can be assumed that the minimum pressure, $\mathrm{P}_{\mathrm{m}}$, is equal to the vapor pressure of the liquid, $\mathrm{P}_{\mathrm{v}}$.

One way to decrease the cavitation number is by decreasing the pressure difference seen in the numerator (only feasible in closed-circuit water tunnels). A second way is to increase the freestream velocity. Initially with a cavitation number greater than the incipient value, $\sigma>\sigma_{\mathrm{i}}$, no cavitation effects are present. As the cavitation number 
drops below the incipient value, $\sigma<\sigma_{\mathrm{i}}$, transient isolated bubbles become apparent. Continuing to lower the cavitation number to values much lower than the incipient value, $\sigma<<\sigma_{\mathrm{i}}$, leads to the development of attached cavities (3D flows) or sheet cavities (2D flows). Attached cavities are seen at the leading edge of a body, while sheet cavities are seen on the low-pressure side of blades and foils. When the cavitation number drops far below the incipient value, $\sigma<<<\sigma_{\mathrm{i}}$, supercavitation and cavitating vortices are formed. Supercavitation can be seen as a vapor-filled cavity encompassing a body underwater or as vortices seen at the tips of 3-D wings or propeller blades. Figure 1.1.2 illustrates the process of decreasing freestream pressure from non-cavitating conditions to supercavitating for a body. As mentioned earlier, supercavitation has advantageous effects, among these is drag reduction allowing for a significant increase in the maximum speed of an underwater vehicle.

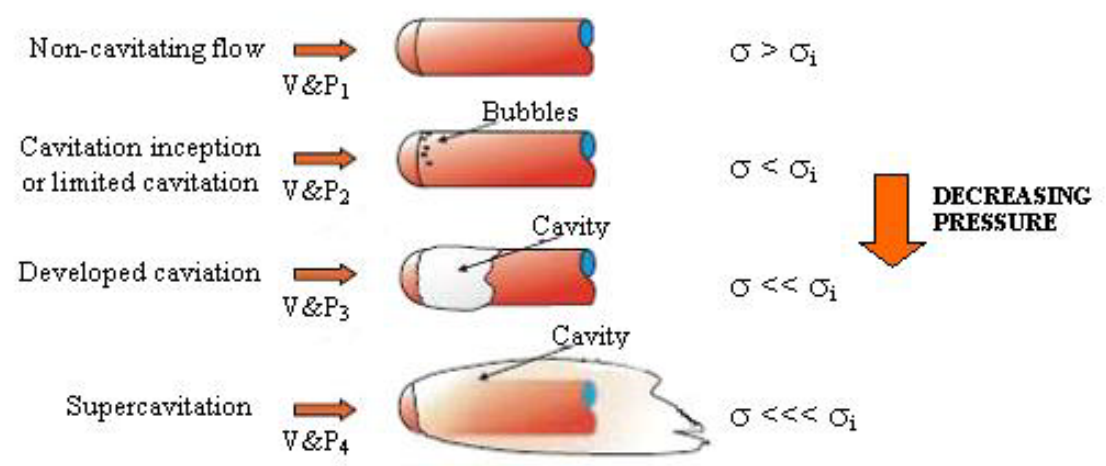

Figure 1.1.2: Cavitation flow regimes (adapted from Stinebring, Billet, Lindau \& Kunz, 2001).

\subsection{Introduction to Supercavitation}

At cavitation numbers much lower than the incipient value, usually below 0.1 , a large, attached cavity can be obtained which can encompass much, and sometimes all, of a body. When supercavitation occurs, the drag of the body surrounded by the cavity is greatly reduced. This is due to the reduction in skin friction drag, which depends on the viscosity of the fluid the body is traveling through. Skin friction drag is much lower for the interaction between a liquid and a vapor than between a liquid and a solid. Natural supercavitation refers to cavitation when a large, attached cavity is created by either an extremely low local freestream pressure, or the body traveling at very high speeds. For a 
natural supercavity, the required cavitation number, usually less than 0.1 , requires a speed in excess of 46 meters per second (>90 knots) at a depth one meter. The required velocity increases with depth (Wosnik et al., 2003).

From an initially non-cavitating flow, the high speeds required for an underwater vehicle to form a natural supercavity are extremely difficult to achieve. There is a second way to create a supercavity around an underwater vehicle. This is done by injecting air into the flow near the front of the body. This method, referred to as artificial supercavitation, was first suggested independently by Reichardt and Epshtein in 19441945 (Epshtein, 1975). The pressure of the injected air into the cavity is then used in place of the vapor pressure of the liquid when determining the cavitation number according to

$$
\sigma_{c}=\frac{P_{\infty}-P_{c}}{\frac{1}{2} \rho U_{\infty}^{2}}
$$

where $\mathrm{P}_{\mathrm{c}}$ is the pressure of the air inside the cavity. This is referred to as the ventilated, or artificial, cavitation number. It is not difficult to see that lower cavitation numbers can be achieved supercavitation can be achieved at much lower speeds by means of artificial cavitation. Natural and artificial cavities are identical at the same cavitation number given that the Froude, Reynolds, and Weber numbers are equal, though this is not often the case (Birkhoff, 1964, Schiebe and Wetzel, 1961).

\subsubsection{Natural vs. Artificial Supercavitation}

There is a significant difference between natural and artificial supercavities that exists in horizontal flows. At a given cavitation number, an artificial supercavity is generated at much lower speeds than the natural supercavity for a given set of conditions. As a result, gravity has a significant role since the supercavity tends to rise due to buoyancy. This makes the Froude number an important parameter when dealing with artificial supercavitation. The Froude number, which is a ratio of the inertial to gravitational effects, is expressed as

$$
F r=\frac{U_{\infty}}{\sqrt{g l}}
$$


where g refers to the gravitational constant and $l$ is a length scale. For current research, the diameter of the cavitator, $\mathrm{d}_{\mathrm{c}}$, is used as the length scale.

Another important parameter for artificial supercavitation is the air entrainment coefficient, which quantifies the amount of gas needed to sustain a ventilated supercavity. This non-dimensional parameter is defined as

$$
C_{q}=\frac{\dot{Q}}{U_{\infty} l^{2}}
$$

where $\mathrm{Q}$ is the volumetric flow rate and $\mathrm{l}$ is once again a characteristic length scale. Once again, the cavitator diameter, $\mathrm{d}_{\mathrm{c}}$, is used as the characteristic length.

Logvinovich (1972) provided a very good summary for the different 'phases' of developed cavitation. He defines his first phase as "weighty" cavitation, when the cavity is formed at low velocities (analogous to artificial cavitation). The third, and final, phase occurs at high speeds when gravity has negligible effects (natural cavitation). The second phase is a transition between the first and final phases. This can occur while an object, initially at low speeds, requiring ventilation to create a cavity, speeds up and begins to approach conditions required for natural supercavitation. Logvinovich (1972) uses these three phases to define boundaries that cannot be exceeded. The lower limit is set by the diameter of the cavitator and is defined as

$$
\sigma_{\min }=\frac{g d_{c}}{U_{\infty}^{2}}=\frac{1}{F r^{2}}
$$

The upper limit is set by the artificial cavitation number. A plot of these limits can be seen below in Figure 1.2.1.1. The focus of this thesis is artificial supercavitation. 


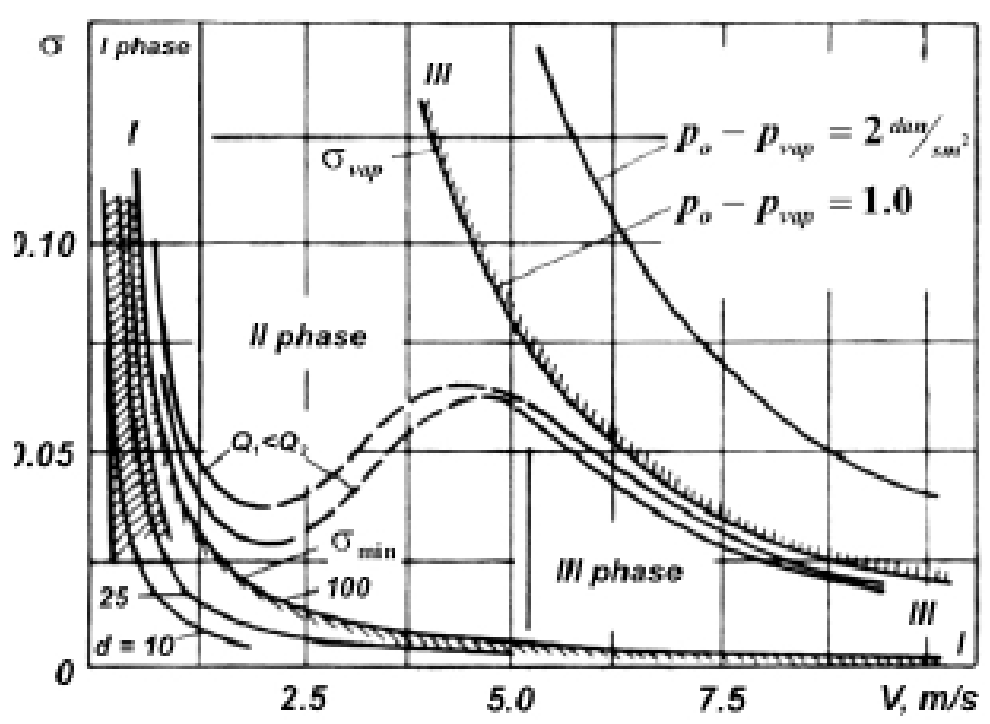

Figure 1.2.1.1: Three phases of developed cavitation (from Logvinovich, 1972).

\subsubsection{Artificial Supercavitation}

Various researchers have conducted experiments as well as numerical simulations on several aspects of supercavities. Semenenko (2001) and Savchenko (2001) pointed out two characteristics that arise from axisymmetric, ventilated supercavities in horizontal flow. The first is the mode of evacuation of air at the rear of the cavity, and the second is the deformation of the cavity under the influence of gravity.

For axisymmetric cavitators, entrainment coefficient and Froude numbers are expressed as

$$
C_{q}=\frac{\dot{Q}}{V_{\infty} d_{c}^{2}}, F r=\frac{U_{\infty}}{\sqrt{g d_{c}}}
$$

Based on simple mass conservation, the flowrate of gas into the cavity must be the same as the flowrate out of the cavity for steady flow. Three modes of evacuation of air have been observed by various authors, which have been summarized by Franc and Michel (2005). In the first mode of evacuation, the tail of the supercavity is filled with foam, which is periodically rejected in the form of unstable, toroidal vortices. This mode is often referred to as the re-entrant jet regime, due to the presence of a re-entrant jet at the tail of the supercavity. This regime is predicted to occur when magnitudes of the Froude 
number and cavitation number are high, i.e. large velocities and short cavities (Franc and Michel, 2005). When this is the case, gravitational effects are negligible and the supercavity shape is nearly axisymmetric.

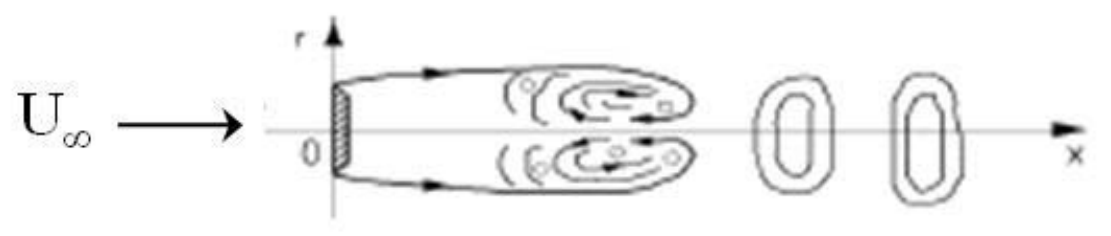

Figure 1.2.2.1: Schematic of re-entrant jet regime. (from Semenenko, 2001).

The second mode of air evacuation is predicted to occur for moderate values of Froude number and small values of $\sigma_{\mathrm{c}}$ (Franc and Michel, 2005). Gravitational effects are considerable, causing the supercavity to curve upwards towards the rear. The tail of the supercavity ends in two hollow vortex tubes, through which gas is continuously evacuated from the supercavity. This mechanism is thus termed the twin-vortex regime. This phenomenon was first described by Cox and Clayden (1956) and later by Epshtein (1971). Due to effects of gravity, the velocity along the surface of the cavity is nonconstant. Using the Bernoulli equation and the assumption that the pressure inside the supercavity, $\mathrm{P}_{\mathrm{c}}$, is constant, the velocity along the top (or bottom) surface of the supercavity is approximated by

$$
U_{ \pm} \cong U_{\infty}-\frac{g z_{ \pm}}{U_{\infty}}
$$

where $\mathrm{z}$ is the height above or below the centerline of the supercavity. The velocity difference between the top and bottom of the supercavity results in a circulation approximated by

$$
\Gamma \cong \frac{g A}{U_{\infty}}
$$

where $\mathrm{A}$ is the intersectional area. This circulation is discharged in two counter rotating trailing vortices at the closure of the supercavity. Campbell et al. (1958) suggested, from empirical considerations, that the first mode of air-entrainment would occur when the product $\sigma \mathrm{Fr}>1$ and the second when $\sigma \mathrm{Fr}<1$. Buyvol (1980) theoretically predicted that 
the twin-vortex regime would occur when $\sigma_{\mathrm{c}}{ }^{3 / 2} \mathrm{Fr}^{2}<1.5$ and the re-entrant jet regime when $\sigma_{\mathrm{c}}^{3 / 2} \mathrm{Fr}^{2}$ was larger than 10 .

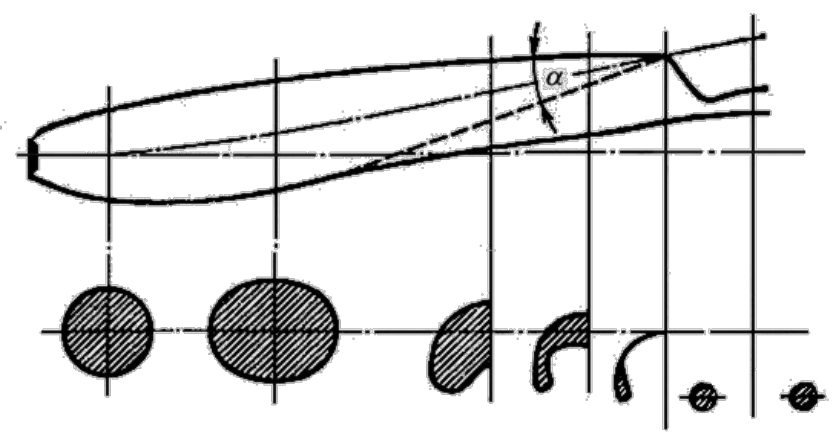

Figure 1.2.2.2: Schematic of twin-vortex mechanism of supercavity closure (Epshtein, 1975)

The third type of gas leakage corresponds to pulsating supercavities. This occurs at high values for the flowrate of gas into the supercavity. Analysis conducted by E.V. Paryshev (1978) showed that the dynamic properties of these supercavities are determined by the ratio between the vaporous and artificial cavitation numbers. This parameter is expressed as

$$
\beta=\frac{\sigma_{v}}{\sigma_{c}}
$$

Supercavities were predicted to be stable when $1<\beta<2.645$ and unstable when $\beta>$ 2.645 .

Kapankin et al. (1984) observed a fourth mode of air evacuation from the tail of the supercavity. This mode consisted of four vortices situated in pairs, one pair above the other. This was predicted to occur when the angle of attack of the cavitator was less than some critical angle determined by

$$
a^{*}=\frac{2 g R_{k}}{3 U_{\infty}^{2}} \frac{1.92-3 \sigma}{\sqrt{c_{x o} \sigma(1+\sigma)}}
$$

where $R_{k}$ is the universal gas constant of the gas and $c_{x o}$ is the drag coefficient of the cavitator when $\sigma=0$. Kapankin seems to be the only author that makes reference to this fourth mechanism of air entrainment. 


\subsection{Supercavitation Advantages}

It is natural to question how the advantages of drag reduction can be applied to underwater vehicles. The possibility for projectiles to travel at extremely high speeds underwater has been area of interest for decades. In the early 1960's the Ukrainian Institute of Hydromechanics began supercavitation research for the development of a self-propelled supercavitating projectile. It took more than a decade to resolve some of the fundamental issues to be solved, including stability. This research led to the development of underwater supercavitating rifles used by the Russian Spetznaz troops. In the mid 1970's the first supercavitating torpedo, the Shkval, or Squall, was developed by the Russian military. This torpedo is believed to have had a range of approximately 5 miles and a top speed greater than $300 \mathrm{mph}$, a speed that would allow the torpedo to sink an opposing submarine without a warhead onboard. The original Shkval is believed to have been unguided, limiting its use simply as a countermeasure from torpedoes fired from opposing stealth submarines. The speed of the supercavitating Shkval was much greater than that of the oncoming torpedo, forcing the opposing stealth submarine to either break off communication with its torpedo by evasive maneuvers, or sacrifice its safety to ensure its target was hit. Exhaust from the jet propulsion system was used as the gas supply to create the artificial supercavity. The Shkval resembled an underwater rocket more so than torpedo. In the 1980's and 1990's a second version of a supercavitating torpedo, the Shkval II has been developed and is believed to be fully guided with a top speed in excess of $450 \mathrm{mph}$ with a much greater range than its predecessor (believed to be around 60 miles) 


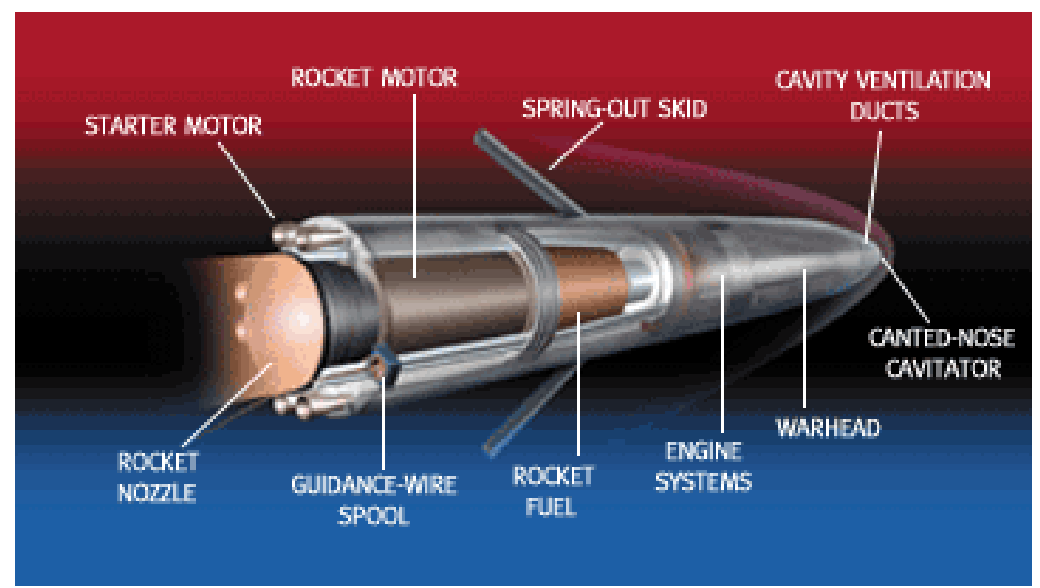

Figure 1.3.1: Shkval torpedo schematic.

Recently, the German company Diehl BGT Defense and Altas Elektronik developed their own version of a supercavitating torpedo. Named the Barracuda, has a top speed over $500 \mathrm{mph}$ and is said to be fully guided, capable of homing in on a target, even if that target is a Shkval torpedo. (Deep Angel Article)

The American company Northrop Grumman utilized supercavitation for underwater mine removal. The system, termed RAMICS (Rapid Airborne Mine Clearance System), is designed to operate from a helicopter where modified bullets from 30mm MK44 Bushmaster II Cannon are fired into the water. The modified bullets, MK 258 Mod 1 armor-piercing, fin-stabilized tracer rounds, stable during flight in air, generate a supercavity upon piercing the surface of the water, allowing it to travel at high speeds until it reaches its target. The bullets use air entrained as a means of creating the supercavity. RAMICS provides a safe and cheap method of surface mine removal for the US Navy. The US Navy has also shown interest in the development of its own, controllable supercavitating torpedo. (RAMICS website) 


\section{DESCRIPTION OF EXPERIMENT}

\subsection{Objectives}

Experiments conducted in water tunnels introduce a factor of blockage that is not present in open water flows. Numerical models have been proposed to predict how blockage imposes limits on experimentation. For some water tunnels, it is possible to design the experiment in such a way that blockage effects are negligible. For other tunnels, where it is not possible to ignore the influence of blockage, it is desirable to know the limitations imposed. For the Saint Anthony Falls Laboratory (SAFL) water tunnel, the test section has a $19 \times 19 \mathrm{~cm}$ test section cross section. In conjunction with ventilation experiments there are supercavitation control experiments (controlling a supercavitating vehicle by means of actuating fins and a cavitator) being conducted by students and staff from the Aerospace Engineering and Mechanics at the University of Minnesota. Currently, the size of the components (sensors and motors) necessary is such that the influence of blockage is unavoidable. The cavitator must be chosen to ensure that the supercavity it large enough to encompass all of model components. Blockage serves to limit the minimum attainable cavitation number due to flow choking. A natural desire is to know how experiments in a water tunnel compare to open water flows. From a cost perspective, it is much cheaper to carry out experiments in smaller water tunnels with a scaled down model than a full scale model in open flow, or even in a large water tunnel. Another goal of current experiments is to find equivalent freestream cavitation numbers for the values found in the water tunnel.

Previous supercavitation experiments were conducted at SAFL by Travis Schauer (Schauer, 2004). Schauer's experiments focused on determining the air entrainment coefficient in the re-entrant jet regime. Unfortunately, due to the re-entrant jet splashing water on the sensors inside the supercavity, Schauer was only able to determine the cavitation number from measurement of supercavity dimensions, determined from the processing of images taken during experimentation. Using results from Brennen's

simulations (1969), Schauer was able to determine a cavitation number from the supercavity's half-length and the cavitator diameter. The aim of the research presented here targets two drawbacks from Schauer's experiments. 
The first drawback is the inability to directly measure the cavitation number. It was believed that the mounting strut for the model was causing the water to splash upstream from the tail of the supercavity onto the model's sensors. To eliminate this effect, the distance from the mounting strut to the cavitator and the cavity pressure measurement location were greatly increased for one model. For the other, it was ensured that the pressure measurement location was upstream of the closure of the supercavity (this will be illustrated later). The results obtained from direct measurement of the pressure inside the supercavity will then be compared to the numerical results from Brennen (1969).

The second drawback arises from an assumption made by Brennen for his numerical model. Brennen assumed an infinite Froude number for his simulation, which is not realistic for water tunnel experiments. A goal of these experiments is to determine the limitations of Brennen's model for water tunnel experiments due to sub-infinite Froude number values tested.

In the 1960's and 1970's, extensive supercavitation research was conducted in Russia. The problem is that not all of this research has been translated into English, and another problem is that very little is known about how these experiments were conducted. This makes it difficult to extract knowledge from their research. Unfortunately, there has been a limited amount of research conducted in the field of axisymmetric supercavitation since 1970. A goal of this paper is verify some of the findings from the Americans prior to 1970 as well as incorporating knowledge gained from the limited amount of Russian literature available on the subject matter.

Very little research has been conducted on the influence of having a body present inside the supercavity. It is not known how the body affects parameters such as the dimensions of the supercavity and the cavitation number. To provide insight into this area of interest, two models were investigated. One model had a body present inside the supercavity while the other did not. 


\subsection{Experimental Facility}

All the experiments were conducted at Saint Anthony Falls Laboratory (SAFL) of the University of Minnesota. The water tunnel, whose schematic can be seen in Figure 2.2.1 is a recirculating, closed-circuit tunnel which can achieve speeds up to $20 \mathrm{~m} / \mathrm{s}$ in the test section. The test section is bounded on three sides by observation windows, which span nearly the entire test section. The test section has width and height measurements of approximately $19 \mathrm{~cm}$ while the length is approximately $1 \mathrm{~m}$ and has circular fillets attached installed in the lower corners. The SAFL water tunnel is unique in the fact that it has a very large gas collector dome upstream of the inlet to the test section, allowing for removal of large amounts of air during ventilation experiments. This allows for ventilation experiments for extended periods of time without circulation of supersaturated water.

1. TEST SECTION $1270 \times 190$ SQ

2. TEST SECTION DOME

3. $7^{\circ}$ DIFFUSER

4. GUIDE VANE ELBOW
5. AXIAL FLOW PUMP $150 \mathrm{HP}$

6. DIFFUSER SCREENS

7. GAS COLLECTOR DOME

8. GAS SEPERATOR, 2134D
9. HONEYCOMB

10. CONTRACTION AND

PARTIAL SHAPE

TRANSACTION

11. NOZZLE

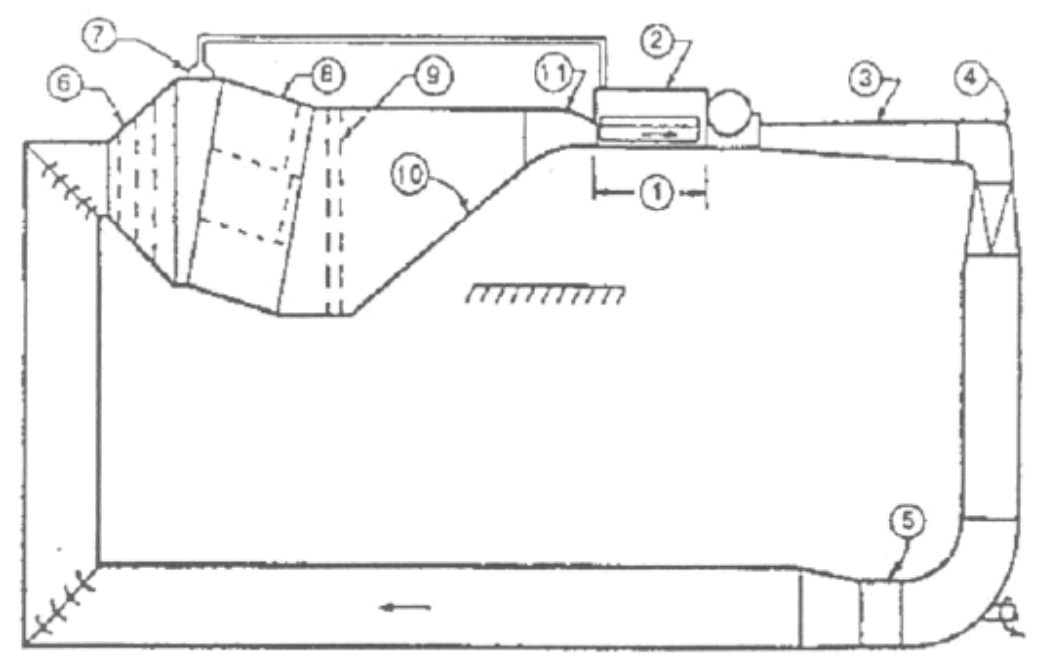

Figure 2.2.1: Schematic of SAFL high speed water tunnel. 
The tunnel is set up in such a way that the absolute pressure can be increased or decreased to a desired level by varying the pressure in a chamber that is attached and located above the tunnel. The static pressure in the test section is measured using an absolute pressure transducer which is connected to a pressure tap in the back window of the tunnel, upstream of the model. The freestream velocity is calculated using a differential pressure transducer which measures the pressure difference between the stagnation pressure in the settling chamber upstream of the test section and the static pressure in the test section (same pressure port as the absolute pressure transducer). Prior to each experiment, the transducers are calibrated using a mercury manometer. Due to the measurement of pressure directly after the outlet of the nozzle connecting the settling chamber to the test section where streamlines are not parallel yet, a correction factor needs to be added when computing the velocity in the test section. Using LDV, the correction factor was found to be 0.960037 and the velocity was computed according to the equation

$$
U_{\infty}=0.960037 \sqrt{\frac{2\left(P_{o}-P_{\infty}\right)}{\rho}}
$$

where $\mathrm{P}_{\mathrm{o}}$ is the stagnation pressure in the settling chamber upstream of the test section, $\mathrm{P}_{\infty}$ is the pressure in the test section and $\rho$ is the density of water.

\subsection{Test Body Design}

The goal of both models was to create a supercavity where the pressure inside the supercavity could be measured directly and accurately. For Schauer's model, the presence of a re-entrant jet due to the presence of the mounting strut, not sufficiently downstream of the cavitator, caused water to splash on the pressure measuring sensors inside the supercavity, causing inaccurate readings. The forward facing model was designed with this in mind. The name 'forward facing model' was chosen due to the fact that the cavitator was upstream of the mounting hydrofoil. As will be shown later, the second model was called the 'backward facing model' because the cavitator was downstream of the mounting hydrofoil. A 0.6 meter long standard $1 / 2$ inch pipe was placed between the mounting strut and the cavitator. To measure the pressure inside the 
cavity, a collar was added which can be observed in the left part of Figure 2.3.1. A barbed fitting was secured to the center of the hollowed out collar which had 3 holes that ran radially outward to the supercavity. 6 holes went around the center of the collar to deliver air to the front part of the model where it was exhausted into a low pressure region behind the cavitator.
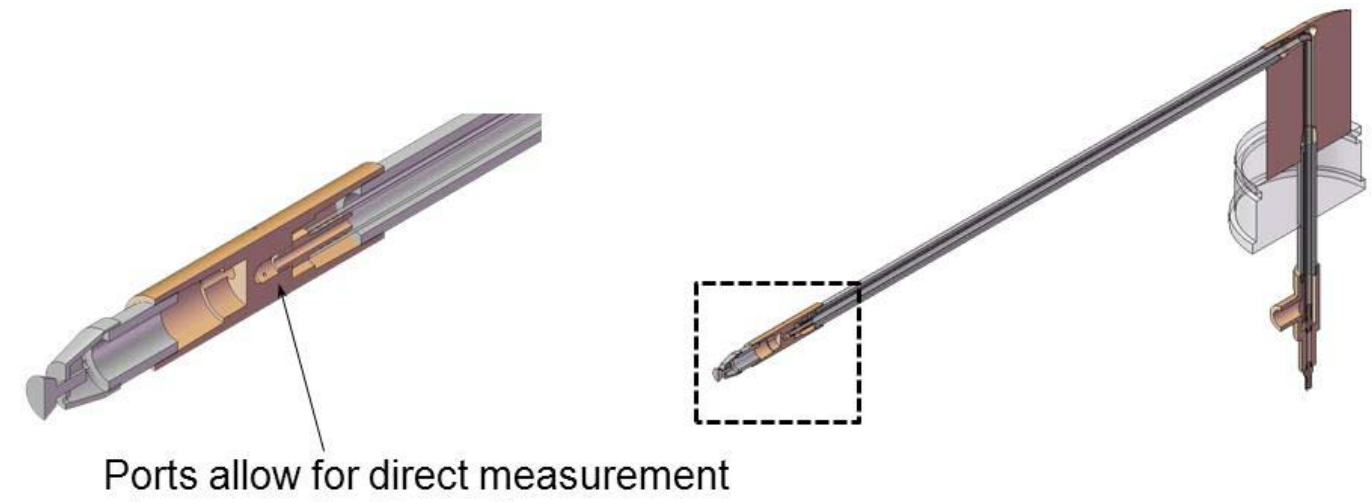

of pressure inside cavity

Figure 2.3.1: Forward Facing Model

The nozzle and cavitator at the front of the forward facing model is shown in Figure 2.3.2. Note that the gas delivery system is slightly different than that observed in Figure 2.3.1. This is for two reasons. The first reason is that the cavitator shown in Figure 2.3.1 is the $10 \mathrm{~mm}$ cavitator. For the $10 \mathrm{~mm}$ cavitator, the gas deflector was too large, causing the supercavity to form on the edge of the gas deflector instead of on the edge of the cavitator. This was only observed for the $10 \mathrm{~mm}$ cavitator and the gas deflector was used for all other sizes of cavitators tested. To remedy this for the $10 \mathrm{~mm}$ cavitator, washers were put in place to deflect the gas radially outward from the injection location. The second reason for the difference between to the two figures is that there were two families of cavitators. One family (Figure 2.3.1) could be screwed directly into the nozzle, while the other family (Figure 2.3.2) attached to a hinge which screwed into the nozzle. This was done with the future plan to replace the hinges with angled edges that would allow for experiments with cavitators at different angles of attack. All the experiments presented here are for a cavitator at a zero-degree angle of attack. 


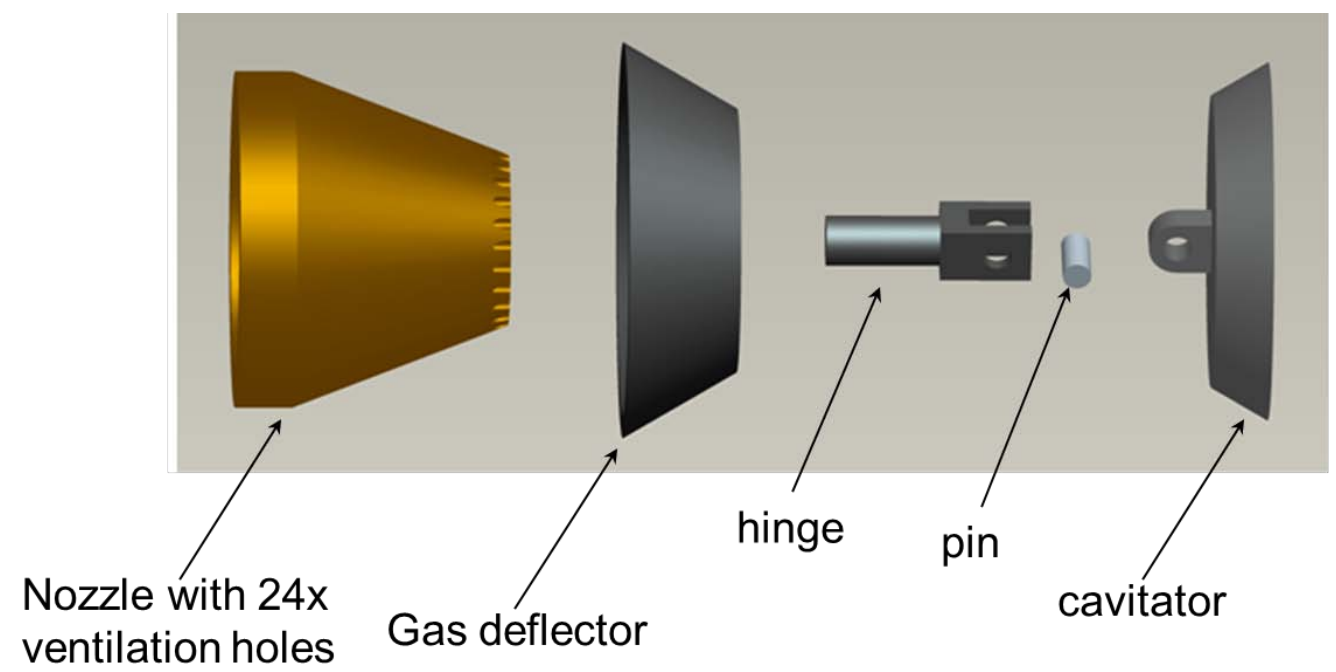

Figure 2.3.2: Forward facing model nozzle and cavitator assembly.

Initial observation of the supercavities created by the forward facing model found the twin-vortex method of cavity closure to be the dominant method for gas removal from the supercavity, different than the re-entrant jet observed by Schauer. It was thought that this might be due to an interaction with the pipe and the surface of the supercavity. As the supercavity was floating up due to buoyancy, the contact with the pipe on either side could cause a circulation which would lead to the formation of the twin-vortex method of supercavity closure.

The backward facing model was designed to investigate if the twin-vortex method of closure was in fact the dominant method, or if it was induced due to interaction with the pipe between the mounting strut and the cavitator. This was done by eliminating the presence of a body inside the supercavity altogether. It will be discussed later that, even without the body present in the supercavity, the twin-vortex closure method was dominantly observed. The backward facing model can be seen in Figure 2.3.3. A difference between the two models was that the location of the pressure measurement location for the forward facing model was perpendicular to the freestream flow, while for the backward facing model, the hypodermic tube was oriented in the same direction as the freestream flow. Assuming that the pressure inside the supercavity was nearly constant, both models’ methods were considered valid. 

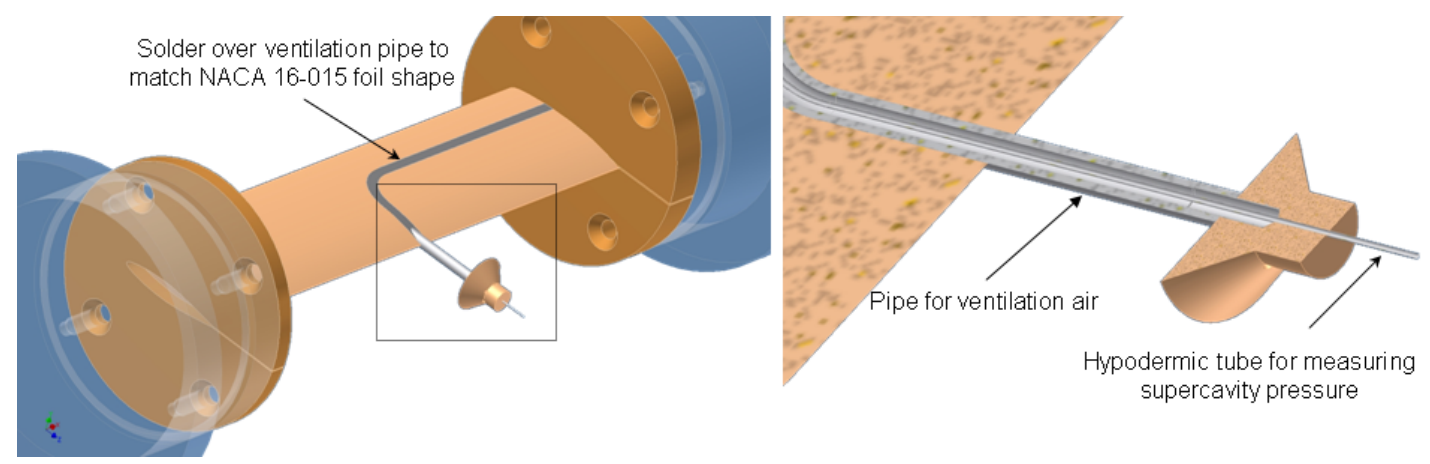

Figure 2.3.3: Backward facing model. Right: Cross section of cavitator and ventilation pipe with hypodermic tube running axially inside.

There were two generations of cavitators that were tested with the backward facing model. The first generation of cavitator, Figure 2.3.4, utilized a porous media to diffuse air that was coming from the supply line into the cavity. It was found that even with the porous media in place, the air exhausted into the supercavity behaved like a jet. The air coming out of the supply line would impinge on the surface of the supercavity at high ventilation rates causing a decrease in the length of the supercavity. It was also found that the hypodermic tube running axially with the supply line was affected by the air being exhausted into the supercavity, making it an inaccurate means of measuring the cavity pressure.
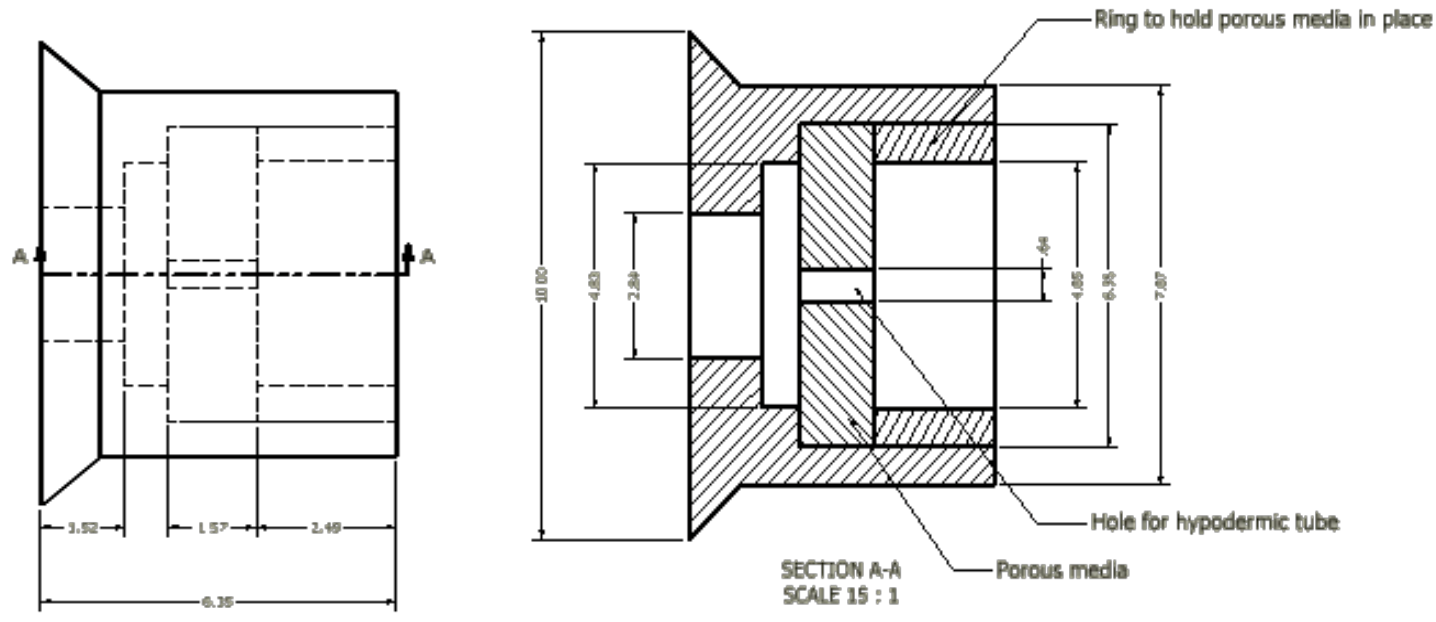

Figure 2.3.4: Backward facing model $10 \mathrm{~mm}$ cavitator, original design. Units in millimeters 
The design for the second generation of cavitator used for the backward facing model can be seen in Figure 2.3.5. To eliminate the jet effect of the air supply impinging on the surface of the supercavity, six holes were inserted at 45 degrees to the supply line. These 6 holes delivered the gas into the flow. The hypodermic tube still ran axially with the flow into the low pressure region behind the cavitator.

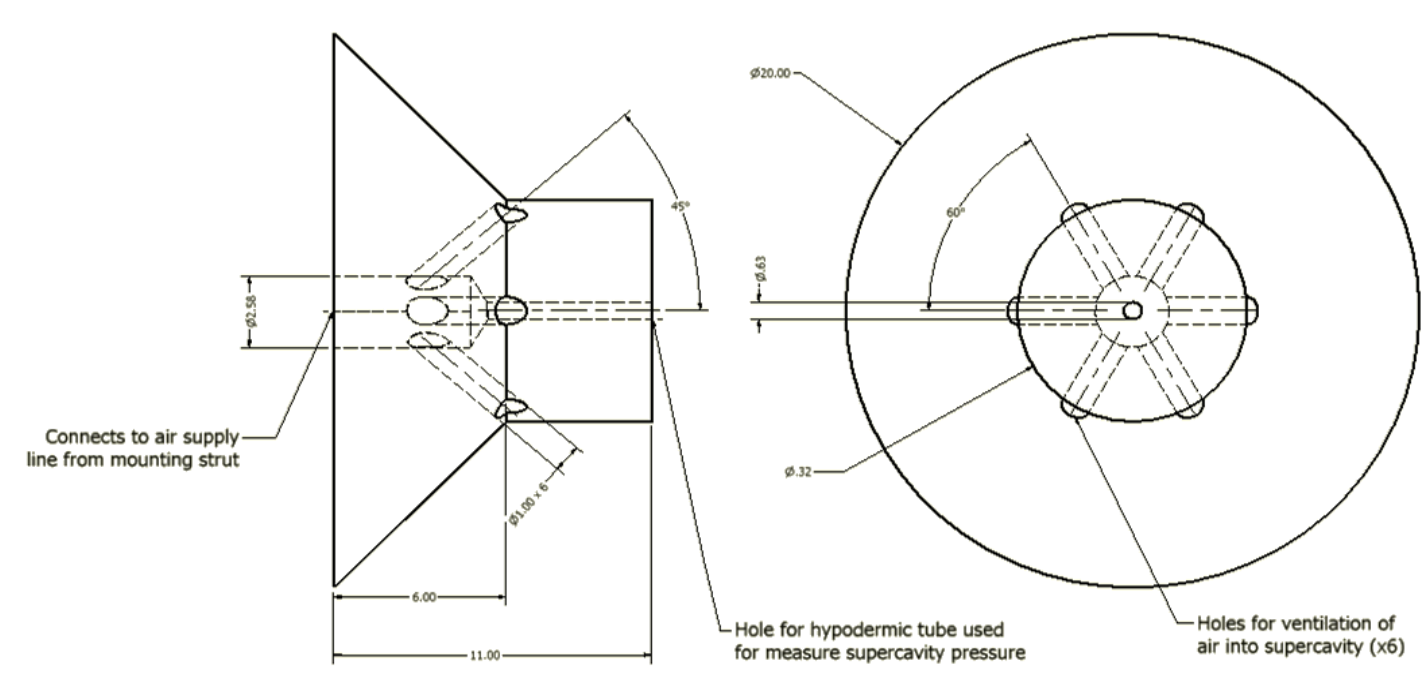

Figure 2.3.5: Backward facing model 20mm cavitator, modified design. Units in millimeters

Interesting effects were observed for both types of cavitators at higher flow rates and low speeds. For moderate flow rates, slightly greater than the flow rate necessary to create a stable supercavity, the surface of the supercavity was smooth and clear aside from the wake signature of the upstream mounting strut. For the jet-type cavitator, as the flow rate increased the supercavity shortened in length due to the impingement of the jet on the surface of the supercavity. The surface of the supercavity also became unstable with large crests developing at high flow rates. This development can be seen in Figure 2.3.6. For the second type of cavitator, as the flow rate increased to high values, much greater than the value needed to create a clear supercavity, visible disturbances could be 
seen on the surface radially outward from the injection locations. Similar to the jet-type cavitator, large crests were observed on the surface of the cavity for very high ventilation rates. These effects can be observed in Figure 2.3.7.

In Figure 2.3.6, corresponding to a jet-type cavitator, in the third image down, a significant decrease in the length of the cavity is already seen when compared to the top image. The only different in conditions between the two images is that the ventilation rate is higher for the third image down. For the images seen in Figure 2.3.7, the second type of cavitator, a decrease is length is not observed with an increase in ventilation rate. Beginning with the fourth image down from the top, visually apparent disturbances are seen on the surface of the supercavity, other than just the wake signature, which are not seen with the jet-type cavitator. These disturbances increase with ventilation rate. For the bottom image in both Figures, significant crests are observed, which suggests that these are attributed more to the high ventilation rate and not the type of cavitator used. 


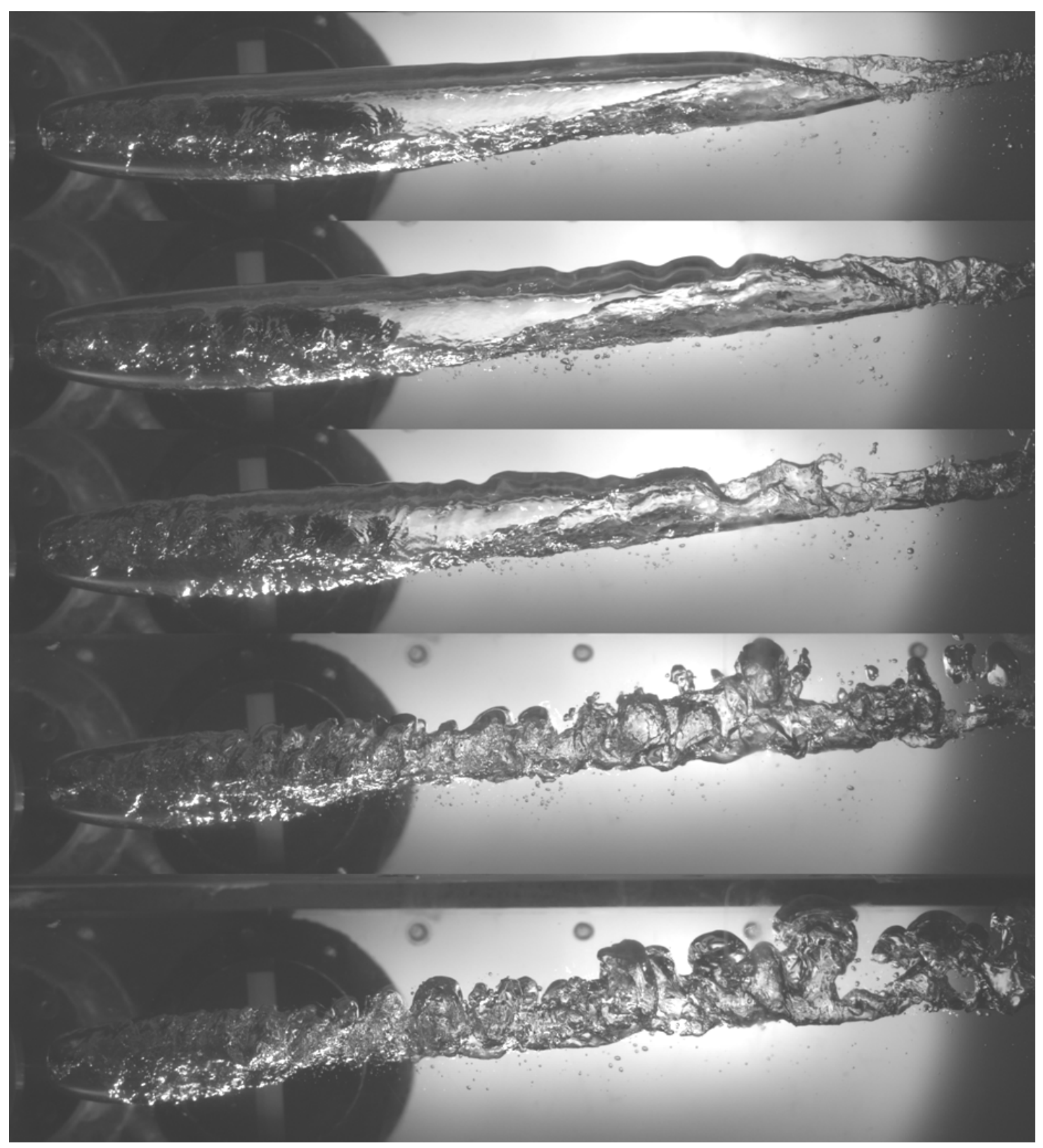

Figure 2.3.6: Supercavity development for the jet-type cavitator. Flow rate increases from top to bottom. Flow velocity is approximately 3.5 meters per second. 


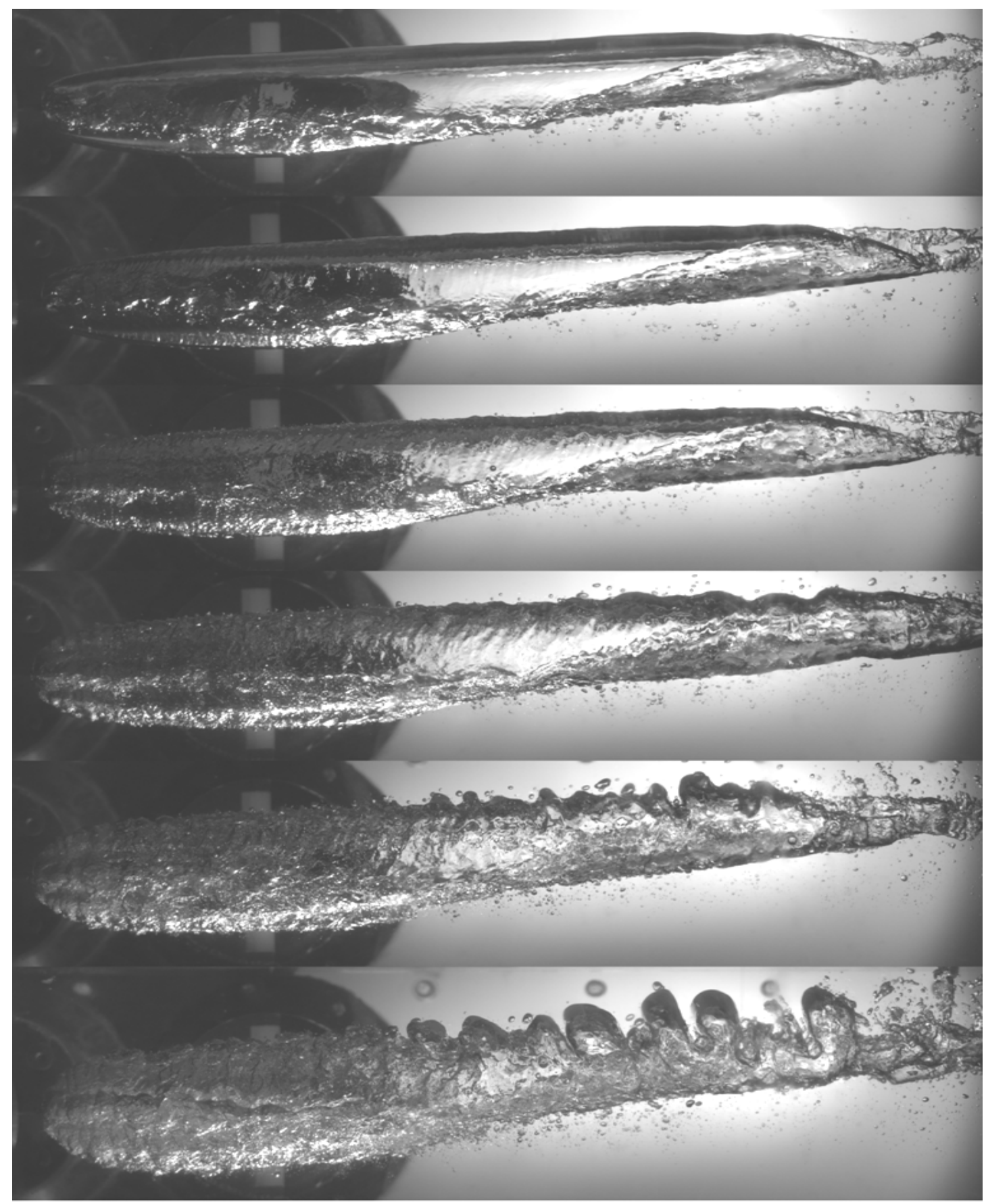

Figure 2.3.7: Supercavity development for the second generation of cavitator. Flow rate increases from top to bottom. Flow velocity is approximately 3.5 meters per second. 
The influence of the presence of the hydrofoil upstream of the cavitator could be visually seen. The wake signature from the NACA 16-015 could be observed on the surface of the supercavity. The surface of the supercavity for the forward facing model was completely clear for a long supercavity, while for the backward facing model the surface of the supercavity in the wake region was not transparent, while the rest of the surface was. A comparison between the supercavities created by the two models can be seen in Figure 2.3.8.
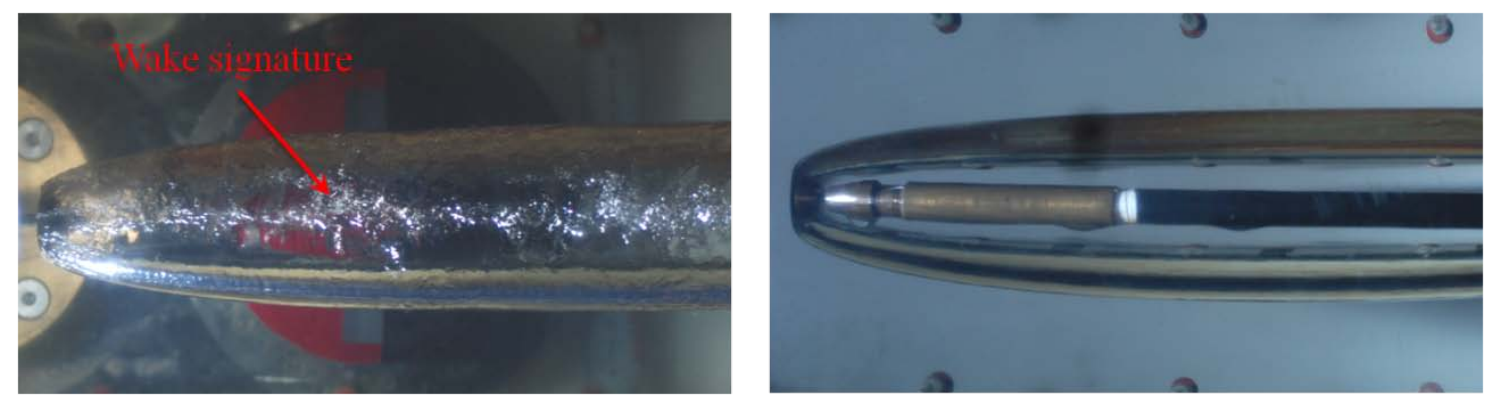

Figure 2.3.8: Comparison of supercavities created by the two models. Left: backward facing model. Right: forward facing model.

For both models, one of the goals was to explore the choking phenomenon encountered when carrying out experiments in a water tunnel. Choking can be easily described by using conservation of mass and the Bernoulli equation for steady, incompressible flow.

Conservation of Mass: $\quad \rho A U=$ constant

Bernoulli: $\quad P+\frac{1}{2} \rho U^{2}=$ constant

The control volume for this analysis can be seen below in Figure 2.3.9. The control volume is shown in red. It is assumed that the pressure inside the supercavity is constant, and the velocity of air is negligible. 


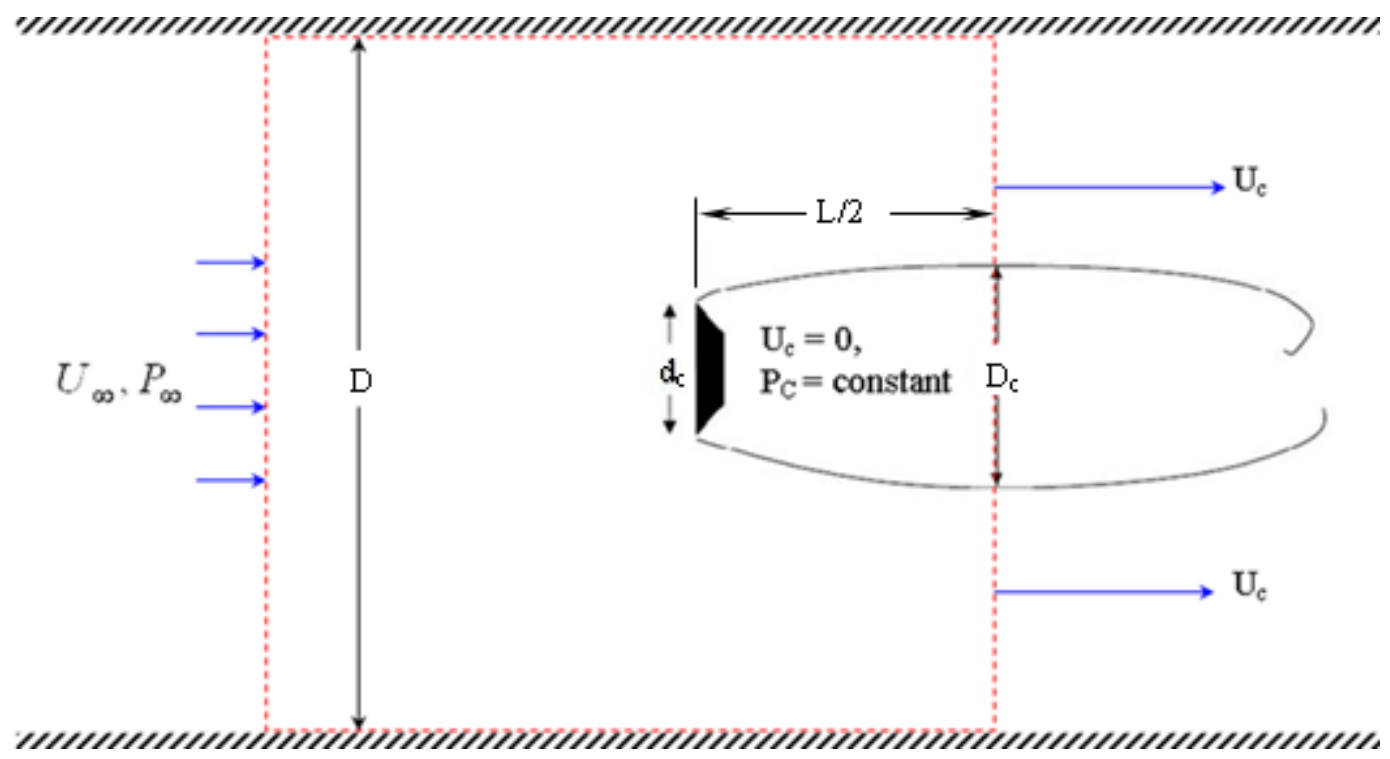

Figure 2.3.9: Problem description for choking phenomenon.

Applying conservation of mass for the control volume, and Bernoulli along a streamline that corresponds to the surface of the supercavity, the following relationships are found

Conservation of Mass: $\quad U_{\infty} A_{O}=U_{c}\left(A_{o}-A_{c}\right)$

Bernoulli: $\quad P_{\infty}+\frac{1}{2} \rho U_{\infty}^{2}=P_{c}+\frac{1}{2} \rho U_{c}^{2}$

Where $A_{0}$ is the cross-sectional are of the test section upstream of the cavitator and $A_{c}$ is the cross sectional area of the supercavity at its maximum diameter, located at the supercavity half-length. Rearranging the Bernoulli equation yields the following relationship

$$
\frac{U_{C}}{U_{\infty}}=\sqrt{1+\sigma_{c}}
$$

Using the conservation of mass equation and the fact that the ratio of cross sectional areas of the supercavity, at its maximum diameter, and the test section (assuming a circular cross section) is equal to the square of the ratio of their diameters $\left(A_{c} / A_{o}=\left(D_{c} / D\right)^{2}\right)$, it is found that 


$$
\frac{U_{c}}{U_{\infty}}=\frac{1}{1-\left(\frac{D_{c}}{D}\right)^{2}}
$$

Finally setting the two equations equal to each other, a relationship is found between the cavitation number and the ratio between supercavity diameter and tunnel diameter.

$$
\sqrt{1+\sigma_{c}}=\frac{1}{1-\left(\frac{D_{c}}{D}\right)^{2}}
$$

Cavity dimensions as a function of cavitation number have been investigated by numerous researchers. Most notably for disc cavitators is the work of Tulin (1961) and Brennen (1969). The continuity equation provides a maximum velocity ratio for a given blockage ratio between the cavity diameter and tunnel diameter. At this value the flow is choked and an increase in the velocity ratio is not possible. Inserting this into the Bernoulli equation, the corresponding cavitation number for the velocity ratio is found. Without an increase in velocity ratio, a decrease in cavitation number is not possible. Note that if no blockage is present, the velocity ratio is one, which would give a cavitation number of zero, as expected. 


\subsection{Experimental Setup}

A schematic of the experimental setup for the forward facing model can be seen below in Figure 2.4.1. Note that while a schematic for the forward facing model is shown below, a similar concept is used for the backward facing model. The tube, or hypodermic pipe, for measuring cavity pressure ran axially with the air supply line that exhausted gas into the supercavity (Refer to Figure 2.3.1 and Figure 2.3.3). Outside of the tunnel, the two were separated by means of a ' $T$ '. Special care was taken to ensure that the air supply line did not affect the line that measured cavity pressure. The cavity pressure, $\mathrm{P}_{\mathrm{c}}$, and freestream pressure, $\mathrm{P}_{\infty}$, were measured using separate Validyne AP 1050 absolute pressure transducers. The velocity was computed using readings from a Validyne DP 15-50 differential pressure transducer. Each pressure reading was recorded by a simultaneous sample and hold board (SS\&H), which was connected to a computer in the lab. The SS\&H board allows for simultaneous sampling of up to 16 channels at rates greater than $5000 \mathrm{~Hz}$. A compressed air line was used as the air supply for the supercavity. The flow rate was measured by a flow meter. For the 10, 15, and 20mm cavitators, an Omega FL-113 rotameter was used. For the 30, 40, and 50mm cavitators, a King flowmeter with range up to $230 \mathrm{l} / \mathrm{min}$ was used. Two different flow measuring devices were used due to the higher gas supply rate required by the larger cavitators to create a clear cavity. The air flow rate was regulated by a valve at the inlet to the flow measuring device. Factory calibrations were used for both flowmeters. During experimentation, special care was taken to ensure that line running to the transducers was purged for accurate readings. For the cavity pressure transducer, air was blown through the line to eliminate unwanted water that may have gotten into the line when a supercavity was not present. For the differential pressure transducer and freestream pressure transducer, unwanted air was purged from the tubing system to ensure accurate readings. 


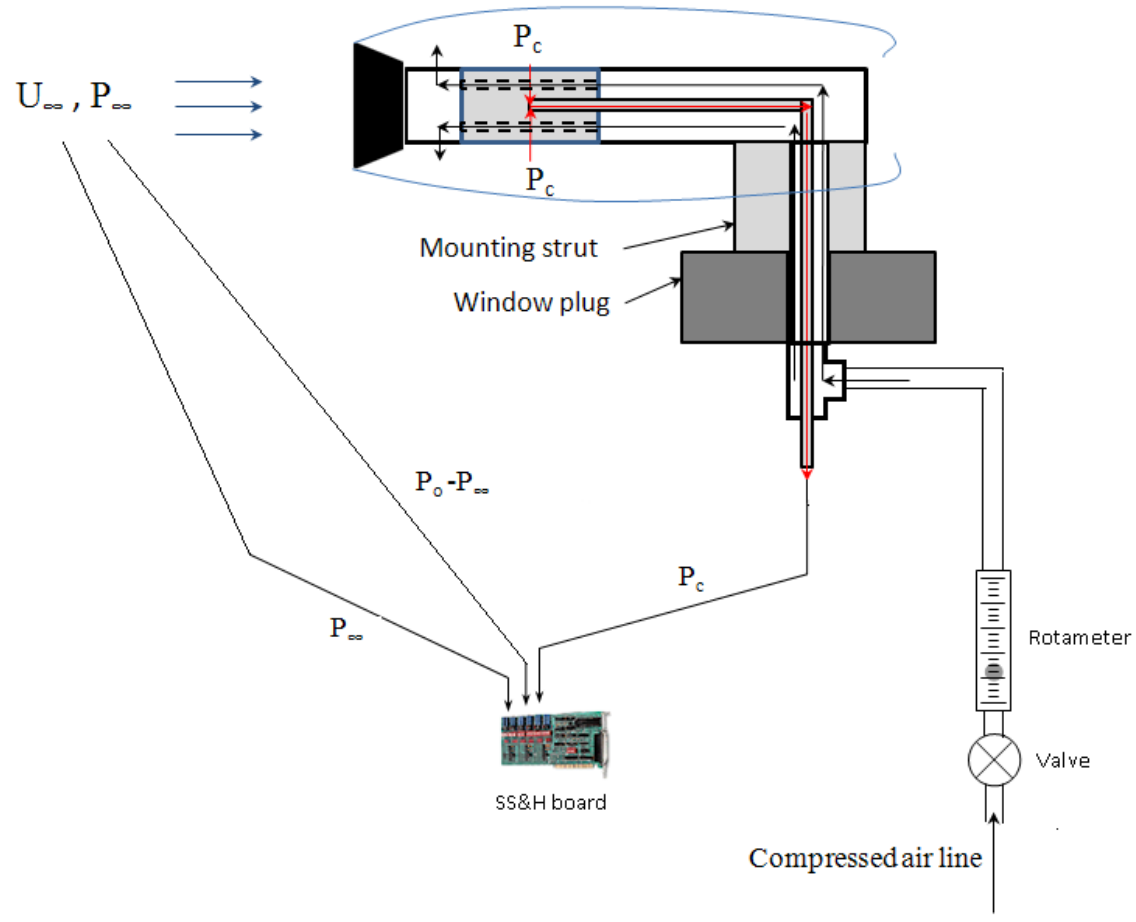

Figure 2.4.1: Experimental setup schematic. Red arrows indicate the path of air from the supercavity to the cavity pressure transducer. Black arrows indicate the path of air from the supply line to the supercavity, unless otherwise noted. (Image not to scale)

The absolute pressure transducer for measuring the static, freestream pressure, $\mathrm{P}_{\infty}$, utilized a flush mounted static port in the rear window of the test section. The differential pressure transducer measures the pressure difference between the settling chamber upstream of the test section and the static pressure in the test section, $\mathrm{P}_{0}-\mathrm{P}_{\infty}$. Calibration procedures for the transducers can be found in Appendix A.

For image acquisition, a Nikon D80 digital camera was used. In order to capture instantaneous snapshots of the flow, strobe lights were used to illuminate the supercavity. The strobes were set at a repetition rate such that the camera shutter was only open for a single pulse. Two IET Strobotac 1531-AB strobes were used and a white light diffuser was used behind the flow in an effort to get uniform lighting. These strobe lights were chosen due to their very short pulse duration, approximately 3 microseconds. Care was taken to ensure that the camera was perpendicular to the flow. This was done to eliminate image distortion which would lead to inaccurate results when processing images to find supercavity dimensions. 


\subsection{Previous SAFL Results}

The study of ventilated flows at SAFL was planned as an interactive experimental/numerical study. The original test body (Figure 2.5.1) studied had a replaceable sharp edged disk that was mounted on the upstream nose of the model. Special ventilation ports for injection of air to create the supercavity were located behind the cavitator. Studies conducted by Wosnik et al. (2003) focused on aspects of the flow physics of the supercavity around the test body. Digital photography was used to investigate the cavity shape and re-entrant jet interaction at various flow conditions. Ventilation requirements to create and sustain a stable supercavity were investigated at various Froude numbers. It was found that the mounting strut for the model, which was downstream of the cavitator, critically affected the ventilation requirements through cavity-strut wake interaction. Blockage effects were also taken into consideration by collecting data for two cavitator sizes, 10 and $15 \mathrm{~mm}$.

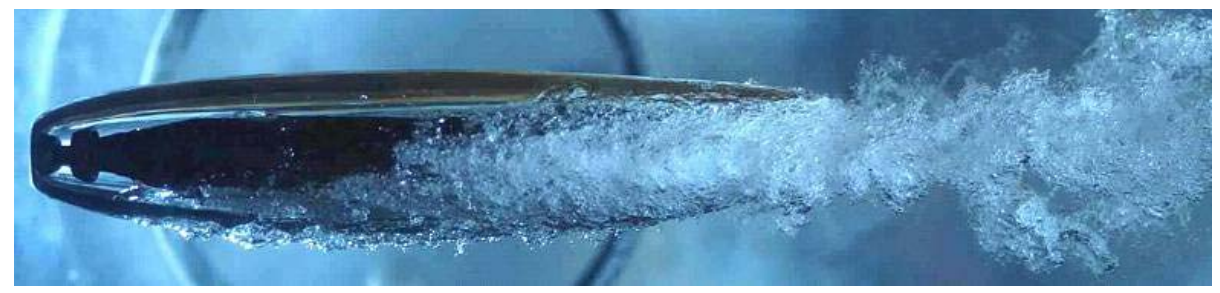

Figure 2.5.1: Original test body studied.

An attempt was also made to directly measure pressure inside the supercavity by means of a pressure transducer that was flush-mounted on the body. These measurements were found to be unreliable due to the non-repeatability of the miniature pressure transducer. This was a result of the re-entrant jet splashing water onto the model. Despite the fact that the pressure could not be measured accurately, the cavitation number could still be determined. From high quality digital images, the supercavity diameter and half-length were determined. These values were then used to find the cavitation number using results from various researchers. It was found that the numerical results from Brennen (1969) were the most useful as they accounted for blockage effects.

Some of the detailed measurements of Wosnik et al. (2003) are shown in Figure

2.5.2. The data shown corresponds to a single Froude number and compares the effects 
of both the cavitator size and the mounting strut type. The data are presented in the form of air entrainment coefficient, $\mathrm{C}_{\mathrm{q}}$, versus the ventilated cavitation number, $\sigma_{\mathrm{c} .}$ It can be seen that the minimum cavitation number for the larger cavitator is higher than for the smaller disk. Also note that at the minimum cavitation number for a given configuration, further increase in ventilation rate did not result in a further decrease in cavitation number. Schauer suggested that this was an effect of blockage. A second consideration is that this behavior is due to the transition from the re-entrant jet regime to the twin vortex regime of air entrainment. The results from Wosnik et al. (2003) provided a basis for the research currently presented.

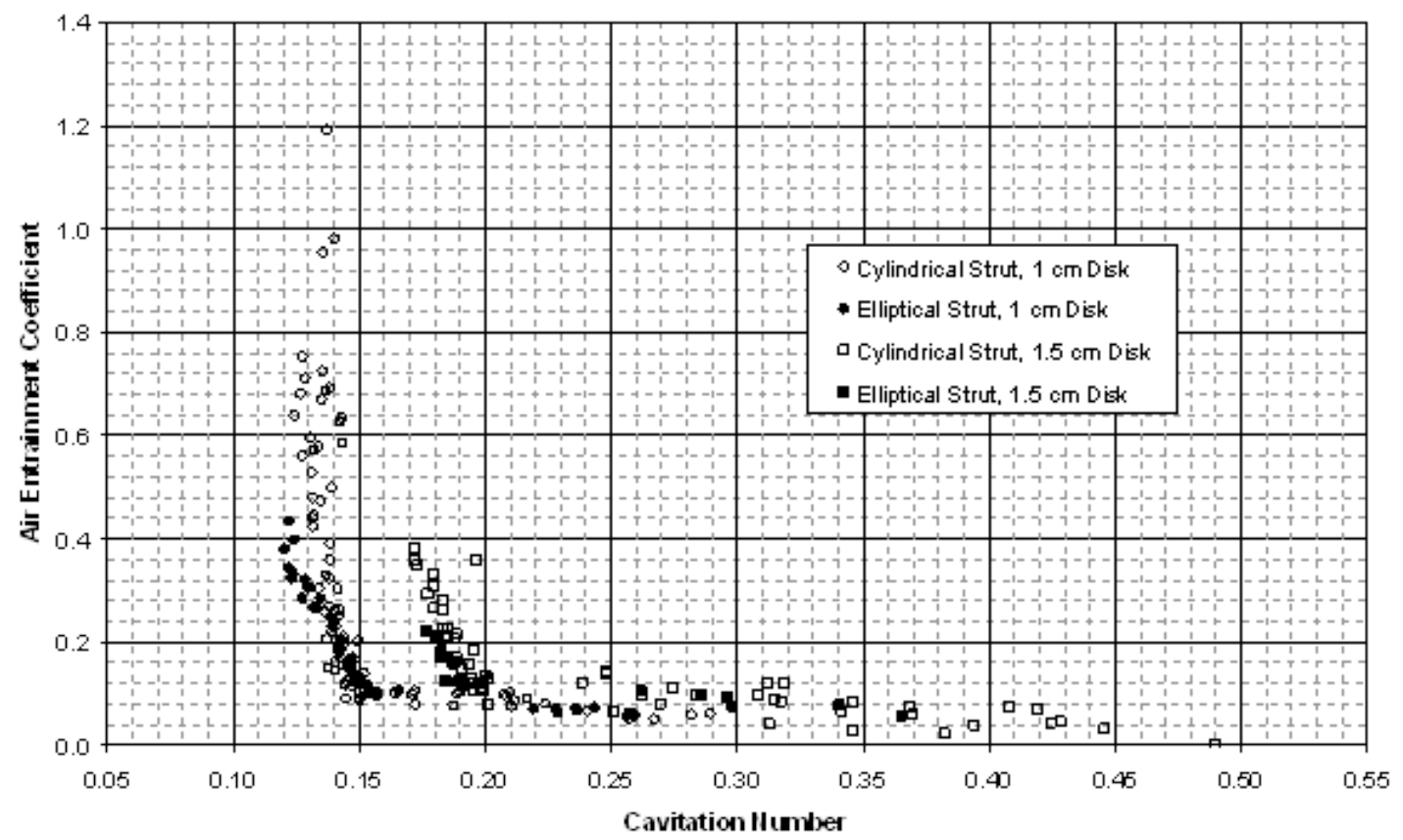

Figure 2.5.2: Air entrainment data for both disks and struts using results from Brennen.

A modified ventilation model was study was conducted by Xu (unpublished) in 2007 at SAFL. The model used for this study investigated two disk cavitator sizes (10 and $15 \mathrm{~mm}$ ) attached upstream of a mounting strut. A significant difference between this model and the original test body studied by Wosnik et al. (2003) was the presence of a long pipe between the mounting strut and the cavitator. The goal of this was to eliminate the influence of the mounting strut on the closure of the supercavity. It was found that as 
the Froude number increased, the length of the supercavity increased, which was an expected result due to the reduced effects of buoyancy. More significantly, a hysteresis effect was observed when the entrainment coefficient was plotted against the cavitation number. Starting from zero ventilation rate, as the ventilation rate increased, the cavitation number decreased to some minimum value, where a twin-vortex supercavity was observed. Once this value was reached, the ventilation rate was decreased, thus lowering the entrainment coefficient. A decrease in entrainment coefficient had no significant effect on the cavitation number until the supercavity collapsed. A typical plot is seen below in Figure 2.5.3. This showed that the amount of air necessary to sustain a supercavity was significantly less than the amount required to form it. Hysteresis is a commonly observed effect in 2D supercavitation, but is not as commonly reported in axisymmetric 3D supercavitation.

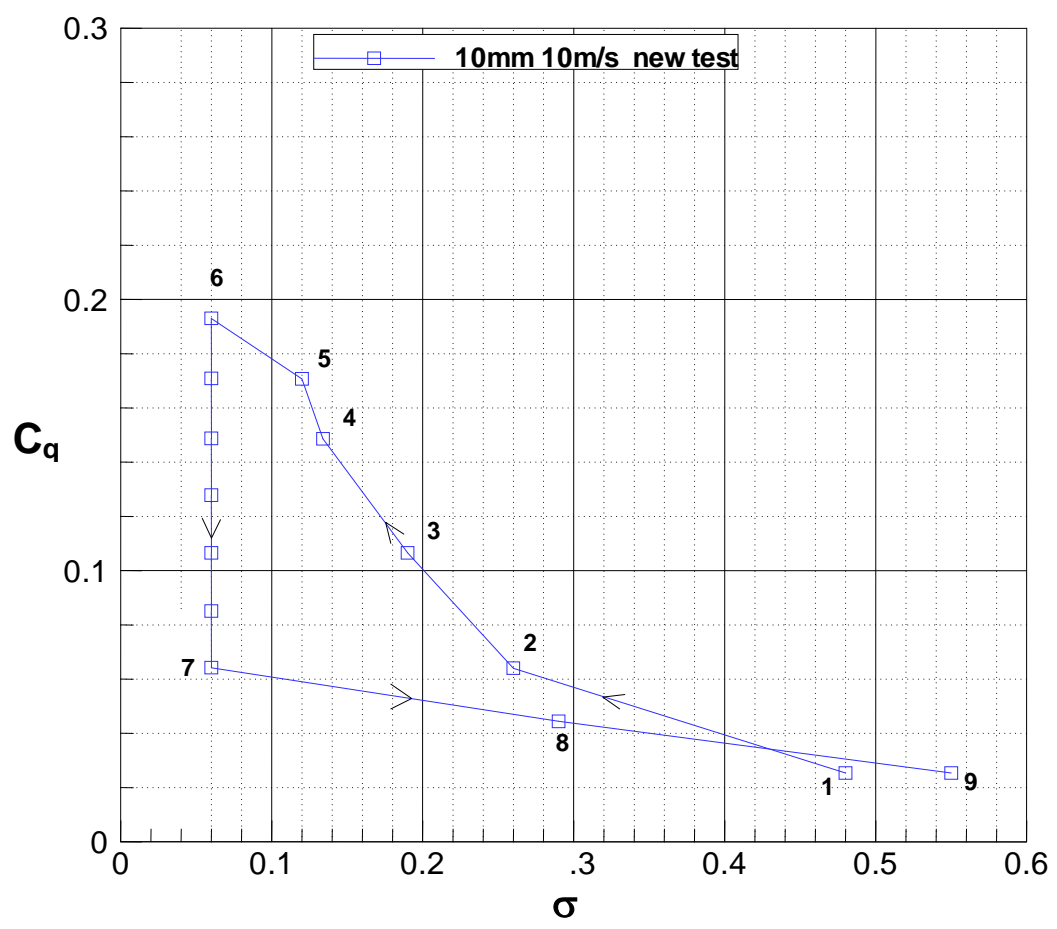

Figure 2.5.3: Typical entrainment plot as observed by Xu (unpublished). Numbers 1-6 indicate an increase in ventilation rate before a stable, twin vortex supercavity has formed. From 6-7 a stable, a twin vortex supercavity is present. From 7-9 the twin vortex supercavity has collapsed and is once again a re-entrant jet supercavity. Note that Brennen's numerical predictions were used to obtain $\sigma$ values. 


\section{DISCUSSION AND EXPERIMENTAL RESULTS}

\subsection{Blockage Considerations}

Typically, underwater vehicles travel in regions where blockage effects are negligible. This is not the case when conducting supercavitation experiments in water tunnels. For water tunnels and channels, the presence of walls or a free surface have significant effects on the dimensions of the supercavity. Logvinovich (1972) noted that upstream of the location of maximum diameter (while the supercavity is expanding) the surface is attracted a free surface. In the rear part of the cavity, where the top of the supercavity has flattened out, the effect of the free surface is to push the cavity away. For walls, the effect is opposite: the supercavity is attracted to the wall toward the tail and pushed away from the wall upstream of the maximum diameter.

For experiments conducted in a water tunnel, blockage has the effect of setting a lower limit on the cavitation number values attainable. Brennen (1969) and Tulin (1961) each conducted numerical simulations which determined the relation between the blockage ratio, $\mathrm{D} / \mathrm{d}_{\mathrm{c}}$, (tunnel diameter to cavitator diameter) and minimum attainable cavitation number, $\sigma_{\min }$. Brennen used a potential flow model to generate a plot of the ratio between the cavitator radius and cavity half-length as a function of the cavitation number for various blockage ratios (Figure 3.1.2). The supercavity half-length is defined as the distance from the cavitator to the point of maximum supercavity diameter. Brennen's ratio of cavitator radius to supercavity half-length can also be expressed as cavitator diameter to supercavity length, assuming that the supercavity length is equal to twice the distance from the cavitator to the location of the maximum cavity diameter. Tulin determined the relationship between the minimum cavitation and the inverse of the blockage ratio (Figure 3.1.1). It should be noted that Brennen and Tulin both assumed an infinite Froude number for their calculations, analogous to an axisymmetric supercavity where buoyancy effects are negligible. A significant difference between the two simulations is that Brennen determines the cavity dimensions as a function of flow choking and cavitation number, whereas Tulin determines cavity dimensions as a function of flow choking only. Brennen's calculations extended to finite and infinite length supercavities, while Tulin's only applied to infinite length supercavities. For an 
infinite length supercavity, the two simulations show good agreement with each other, as expected. Both found that as the blockage ratio between the test section diameter and cavitator diameter decreased (i.e. the cavitator size increased) the minimum attainable cavitation number increased.

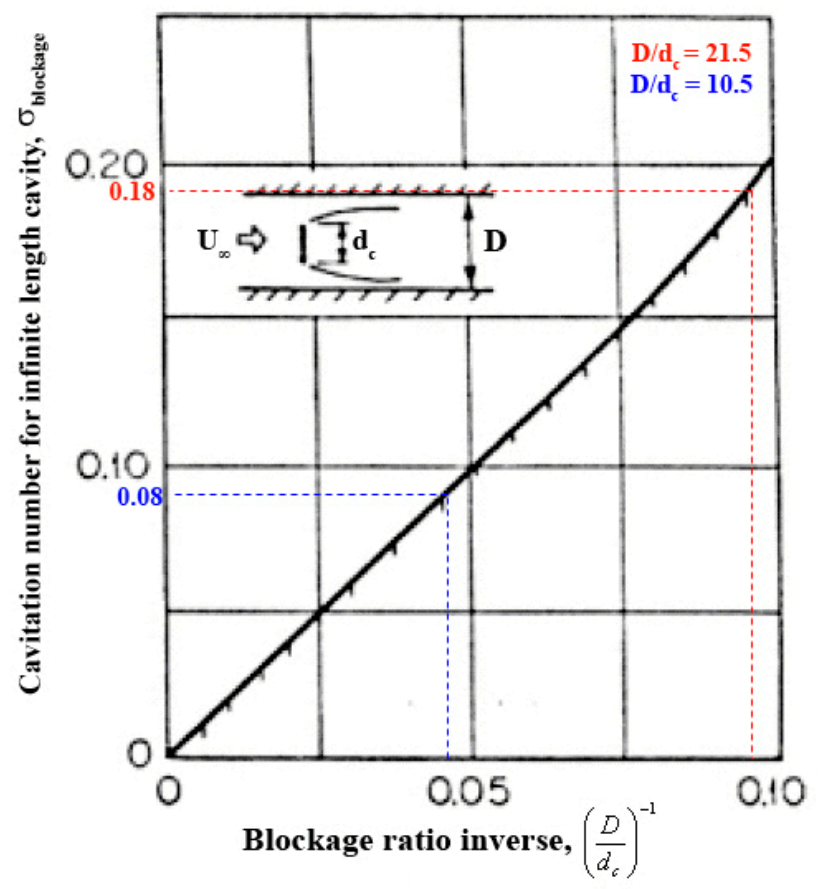

Figure 3.1.1: Tulin (1961) predictions for minimum cavitation number as a function of the inverse of the blockage ratio. $\mathrm{D} / \mathrm{d}_{\mathrm{c}}=21.5$ and $\mathrm{D} / \mathrm{d}_{\mathrm{c}}=10.5$ correspond to cavitator diameters of $10 \mathrm{~mm}$ and $20 \mathrm{~mm}$ respectively.

Table 3.1.1 provides a summary of the various cavitator sizes test for the models and their corresponding minimum cavitation values obtained from Brennen's plot (Figure 3.1.2). Brennen's original plot did not have a curve corresponding to a $15 \mathrm{~mm}$ cavitator but Schauer used a cubic spline interpolation to generate one. For the forward facing model, all the cavitators shown in Table 3.1.1 were tested. For the backward facing model, only the $10 \mathrm{~mm}$ and $20 \mathrm{~mm}$ cavitators were tested. Experimental results can be seen in Figure 3.1.2. For each size cavitator, velocities from 3-10 m/s were tested. Note that only cavitation numbers determined from direct measurement of cavity pressure are shown. This means that only results from long, clear cavities are shown (twin-vortex regime). 
Table 3.1.1: Summary of blockage ratio, $D / d_{c}$, values and corresponding minimum cavitation values as predicted by Brennen (1969).

\begin{tabular}{|c|c|c|c|c|}
\hline \multirow{3}{*}{$\begin{aligned} \mathrm{d}_{\mathrm{c}} & =10 \mathrm{~mm} \\
\mathrm{~d}_{\mathrm{c}} & =15 \mathrm{~mm}\end{aligned}$} & \multirow{2}{*}{$\frac{\mathrm{D} / \mathrm{d}_{\mathrm{c}}}{21.3}$} & \multicolumn{3}{|c|}{ From Brennen's Plot } \\
\hline & & $\sigma_{\min }=$ & 0.08 & for $h / d=21.5$ \\
\hline & 14.2 & $\sigma_{\min }=$ & 0.147 & for $h / d=14.3$ \\
\hline $\mathrm{d}_{\mathrm{c}}=20 \mathrm{~mm}$ & 10.65 & $\sigma_{\min }=$ & 0.202 & for $h / d=10.5$ \\
\hline $\mathrm{d}_{\mathrm{c}}=30 \mathrm{~mm}$ & 7.1 & $\sigma_{\min }=$ & 0.295 & for $h / d=7.5$ \\
\hline $\mathrm{d}_{\mathrm{c}}=40 \mathrm{~mm}$ & 5.325 & $\sigma_{\min }=$ & 0.495 & for $h / d=5.0$ \\
\hline $\mathrm{d}_{\mathrm{c}}=50 \mathrm{~mm}$ & 4.26 & $\sigma_{\min }=$ & 0.596 & for $h / d=4.4$ \\
\hline
\end{tabular}

For the forward facing model, good agreement is found between Brennen's predictions and experimental results for most of Froude numbers tested. For a given cavitator, at the lower Froude numbers, the cavitation number tended to be slightly greater than the value predicted by Brennen. As Froude number increased, there was better agreement with predictions. This was not surprising, since Brennen's calculations assumed an infinite Froude number. It is surprising that only at low Froude numbers, corresponding to velocities in the range of $3-5 \mathrm{~m} / \mathrm{s}$, was there a significant diffefence with Brennen’s results.

The backward facing model showed significant differences with the results from the forward facing model. For the backward facing model, agreement with Brennen was only good at high Froude numbers, corresponding to velocities in excess of $10 \mathrm{~m} / \mathrm{s}$, for the cavitators tested. Starting at low Froude numbers, associated with $3 \mathrm{~m} / \mathrm{s}$, agreeement was significantly different than Brennen's predictions. As the Froude number increased, the experimental data showed more agreement with Brennen. For the backward facing model, much higher Froude numbers were necessary to utilize Brennen's predictions for determining cavitation number. It will be shown later that the Froude numbers needed for the backward facing model to use Brennen's predictions correspond to an approximately axisymmetric supercavtiy, an assumption made by Brennen. 


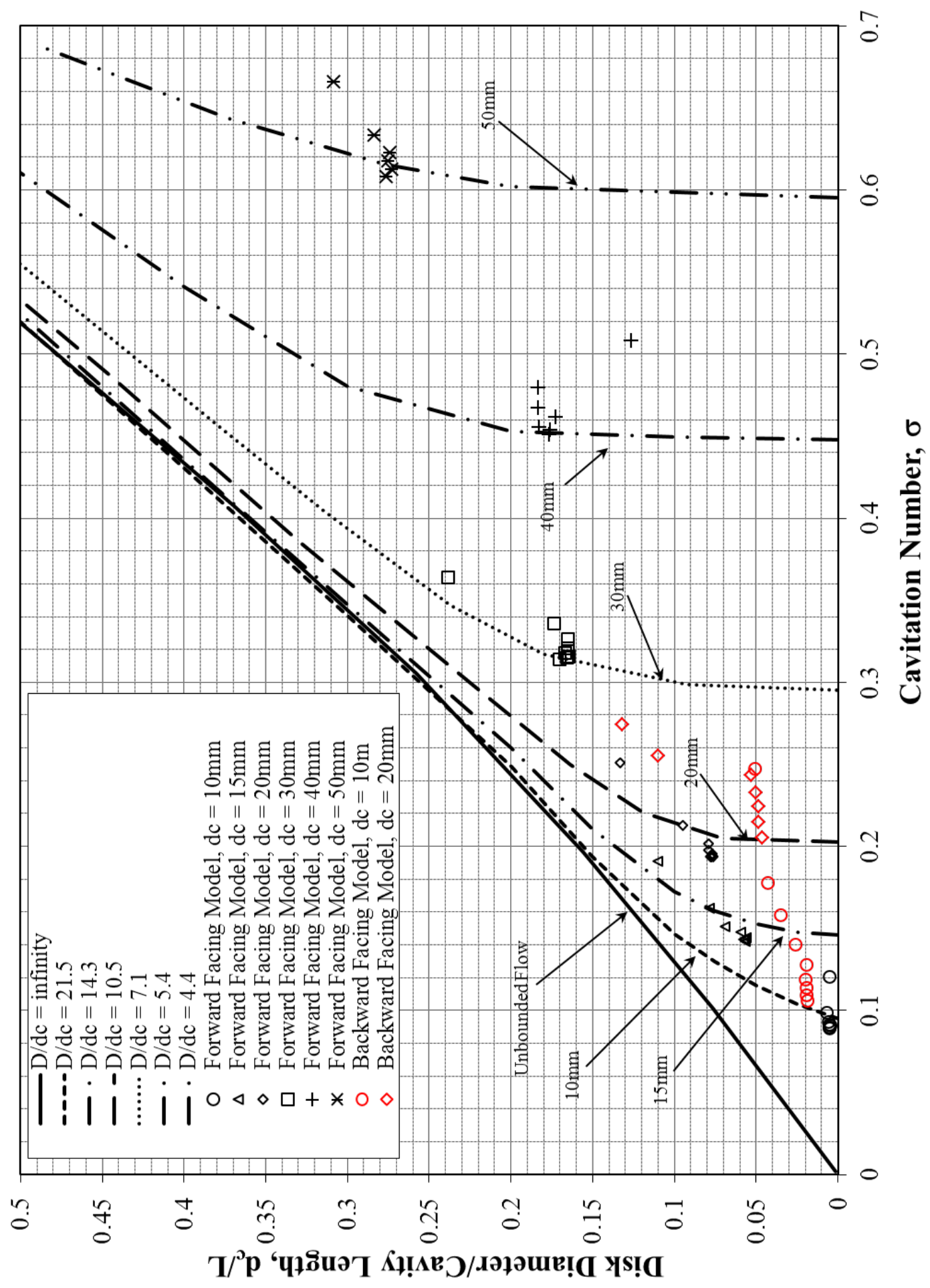

Figure 3.1.2: Experimental data compared to Brennen's numerical predictions for both models. Results from the forward facing model are shown in black, while results from the backward facing model are shown in red. Calculations from Brennen (1969) are shown by lines while experimental data is shown as points. 


\subsection{Air Entrainment}

Air entrainment behind an artificial supercavity was first experimentally investigated at the California Institute of Technology by Swanson and O’Neill (1951). Their supercavitation experiments were conducted in a free surface water tunnel. A summary of their results can be seen in Figure 3.2.1. Given a cavitator size, the reentrant jet regime was found to be present above some critical value of cavitation number. In this region, little change is seen in the entrainment coefficient with a change in cavitation number. Below this critical cavitation number, the twin-vortex regime was found to be dominant, where a large increase in ventilation was necessary for a small change in cavitation number. It should be noted that as the cavitation number decreased the entrainment coefficient decreased until it reached the critical value where a transition between the re-entrant jet regime and twin vortex regime was found to occur. Similar results were seen by Semenenko (2001). Other researchers (Spurk, 2001) predicted, using numerical models, the entrainment coefficient to continually increase with a decrease in cavitation number (Figure 3.2.2). It should be noted that Spurk made an assumption of very large Froude numbers, Fr $>>1$, for his numerical model. This will be discussed further later.

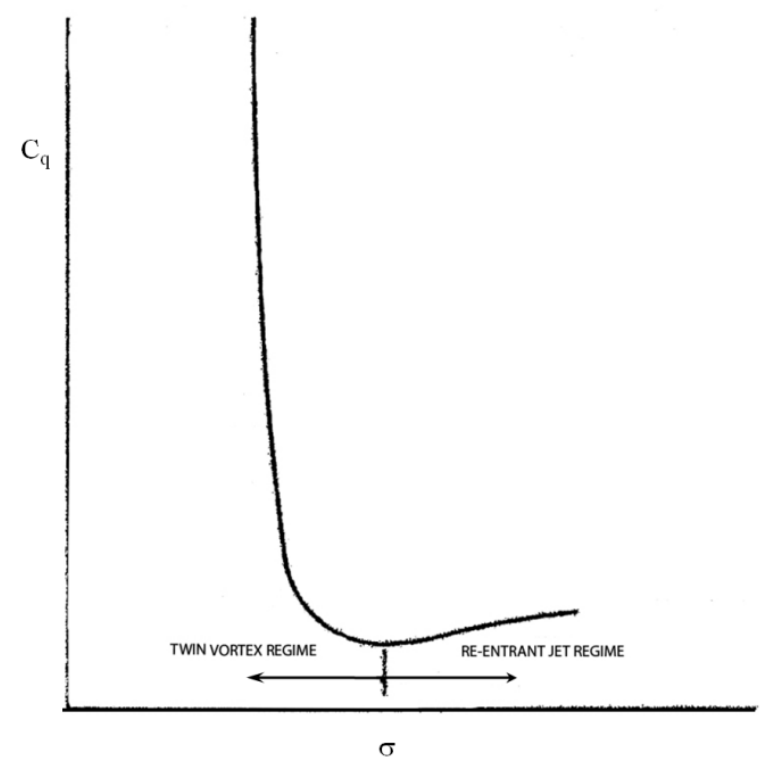

Figure 3.2.1: Typical entrainment curve, obtained experimentally, for a cavity formed behind a given disc at a given speed (Campbell and Hilborne, 1958, originally from Swanson and O’Neill, 1951). 


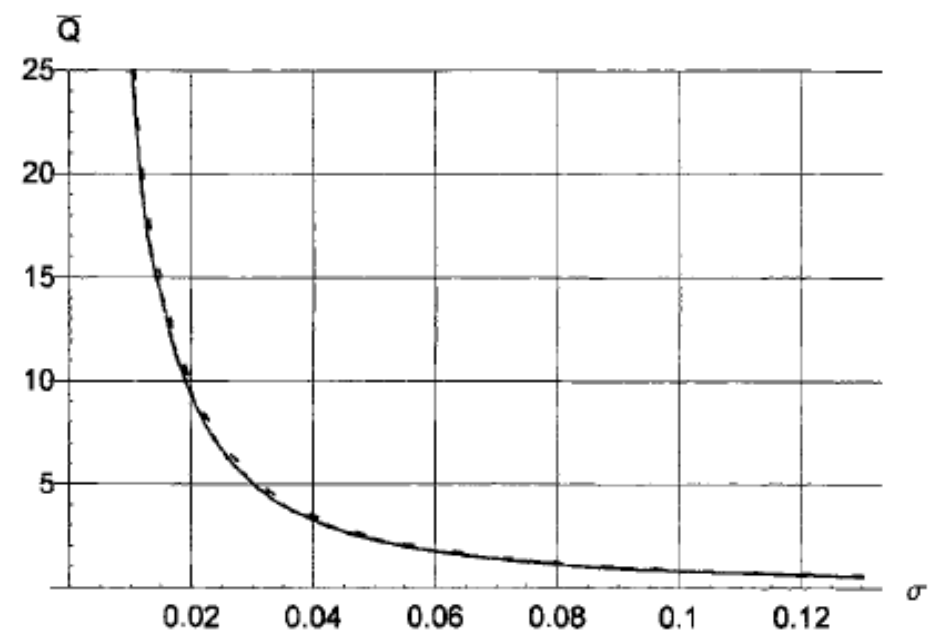

Figure 3.2.2: Modified form of entrainment coefficient plotted against cavitation number as predicted by Spurk's numerical model. Solid line: $\mathrm{C}_{\mathrm{D} 0}=0.80$. Dashed line: $\mathrm{C}_{\mathrm{D} 0}=$ 0.151. (Spurk, 2001)

May (1975) noted that while various researchers have studied the re-entrant jet regime, little agreement is found. He attributes this to the variation in conditions at which the experiments took place.

Figure 3.2.3 and Figure 3.2.4 show the stages of supercavity development from a short, foamy cavity to a long, clear supercavity. For these experiments, the ventilation rate was increased in step increments, gathering data and images at each step. At some critical value for the entrainment coefficient, depending on the Froude number, the supercavity would transition very rapidly from a short, foamy cavity (re-entrant jet) to a long clear supercavity (twin-vortex regime). This behavior was observed for both models. A possible explanation for this behavior with the forward facing mode is provided by Semenenko (2001). Although it is tempting to extend his explanation to the backward facing model, where similar behavior is observed, Semenenko's theory is for a supercavity with a body inside. His explanation does not account for the hysteresis effect seen with both models where little variation of cavity half-length is seen with a decrease in ventilation after the formation of a clear supercavity. It should be noted that transducer data is available only when a clear supercavity is present. This is due to the unsteady 
nature of a short, foamy cavity, as well as water splashing on the pressure measurement location for both models.

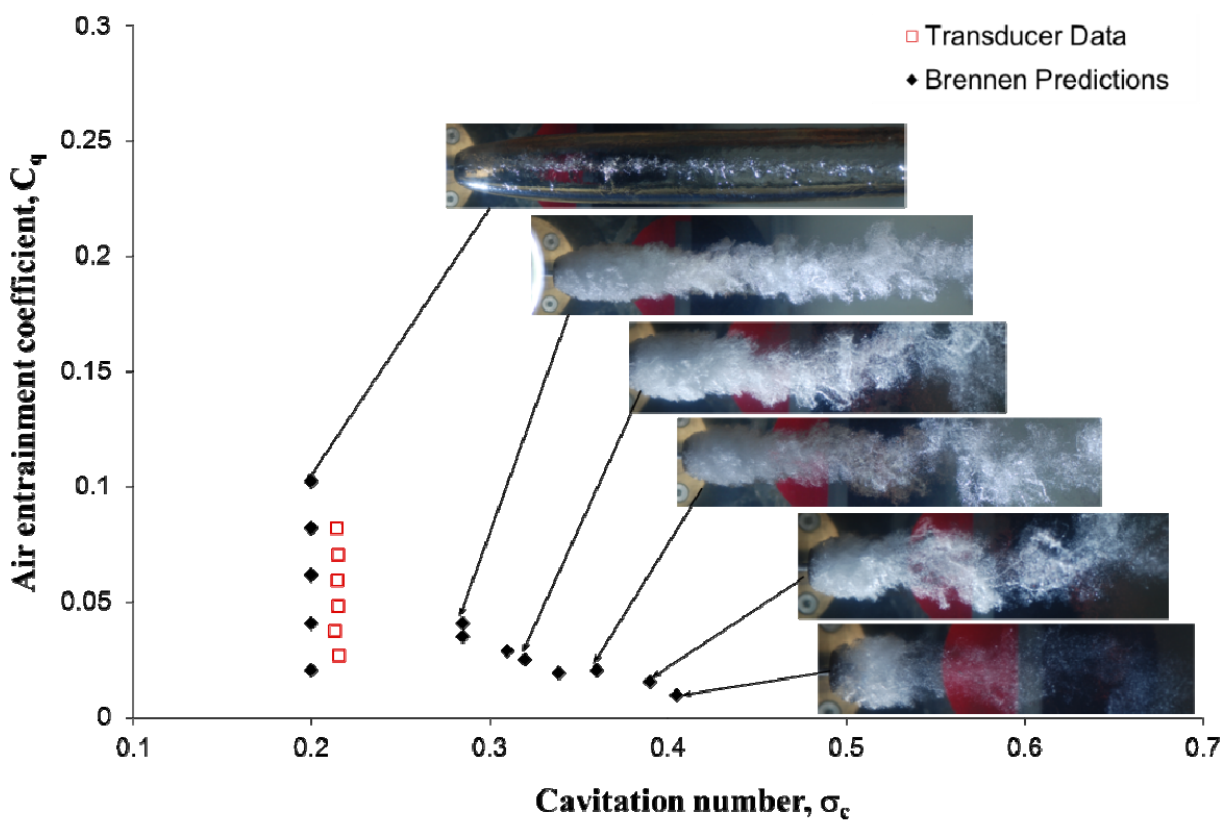

Figure 3.2.3: Backward facing model cavity development. Note that transducer data is not available when a clear cavity is not present. $\mathrm{Fr}=23, \mathrm{~d}_{\mathrm{c}}=20 \mathrm{~mm}$.

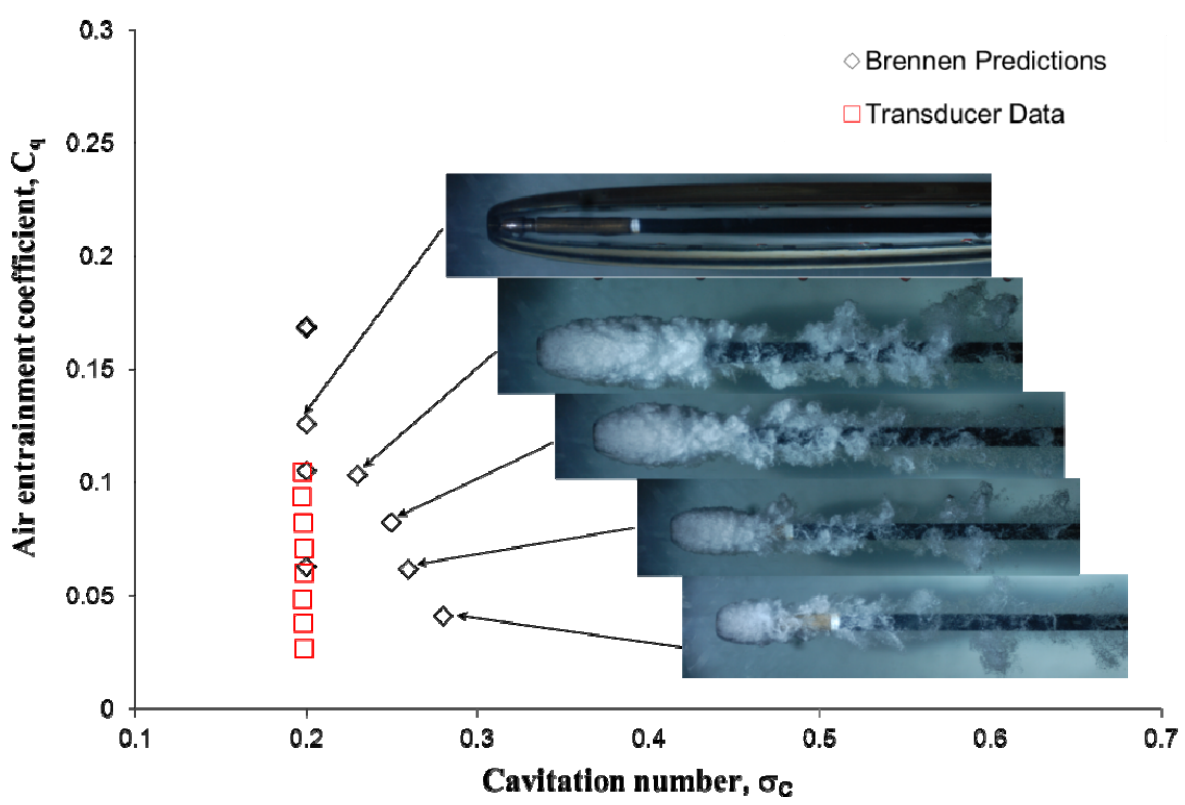

Figure 3.2.4: Forward facing model cavity development. Note that transducer data is not available when a clear cavity is not present. $\mathrm{Fr}=23, \mathrm{~d}_{\mathrm{c}}=20 \mathrm{~mm}$. 
Before the transition to the twin-vortex regime, the cavitation number was determined from processing of the images taken during the experiment. Using a Matlab script developed by Dr. William Hambleton (Appendix C), the supercavity half-length and maximum diameter were determined by taking the average over 2-3 images. These values were then used with Brennen's plot (Appendix C) to determine the cavitation number. As the ventilation rate increased before the transition value, an increase in ventilation rate corresponded to a decrease in cavitation number. Once the transition value was reached and a long, clear supercavity was formed, an increase in ventilation had little effect on the cavitation number. A large increase in ventilation was necessary for a small change in cavitation number, similar to results from Swanson and O’Neill (1951). It was also observed that once the transition value had been exceeded, the ventilation rate could be decreased below the transition value without a collapse of the supercavity and little effect on the cavitation number. This is illustrated in Figure 3.2.3 and Figure 3.2.4 where transducer data are available for entrainment coefficients lower than the transition value. Essentially the cavitation number reaches a minimum once a clear supercavity is formed. The transducer data and the results from Brennen's numerical predictions show good agreement once a clear supercavity has been established for certain values of Froude number. Recalling the earlier discussion regarding the Froude number necessary to use Brennen's plot for either model, good agreement was only found, as expected, between the transducer data and Brennen's predictions once a certain Froude number was reached, depending on the model. 
Keeping in mind that Brennen and Tulin's calculations assumed an axisymmetric supercavity, or infinite Froude number, it is interesting to find agreement with experiments where the supercavity is influenced by buoyancy making the supercavity non-axisymmetric, at finite Froude numbers. A natural desire is to find out when calculations for an axisymmetric supercavity can be applied to a non-axisymmetric one. Figure 3.2.5 illustrates a ratio of cavitation numbers, $\sigma_{\mathrm{c}} / \sigma_{\min }$, plotted against Froude number, where $\sigma_{\min }$ is the minimum cavitation number predicted by Brennen and Tulin for an infinite length, axisymmetric supercavity. When the ratio between cavitation numbers was close to one, there was good agreement between experimental data and the numerical predictions of Brennen and Tulin. At lower Froude numbers, there was significant disagreement between experimental data and numerical predictions. This is not surprising, given that the ratio between the two cavitation numbers is much greater than one. As Froude number increased, agreement increased. The reason why the $20 \mathrm{~mm}$ cavitator has values for a cavitation number ratio slightly less than one for higher Froude numbers is attributed to the fact that blockage ratio for the $20 \mathrm{~mm}$ cavitator was approximately 10.2, which did not exactly match the blockage ratio of 10.7 from Brennen. Figure 3.2.5 also shows that for a non-axisymmetric supercavity, the cavitation number corresponding to a clear supercavity is function of blockage and Froude number. 


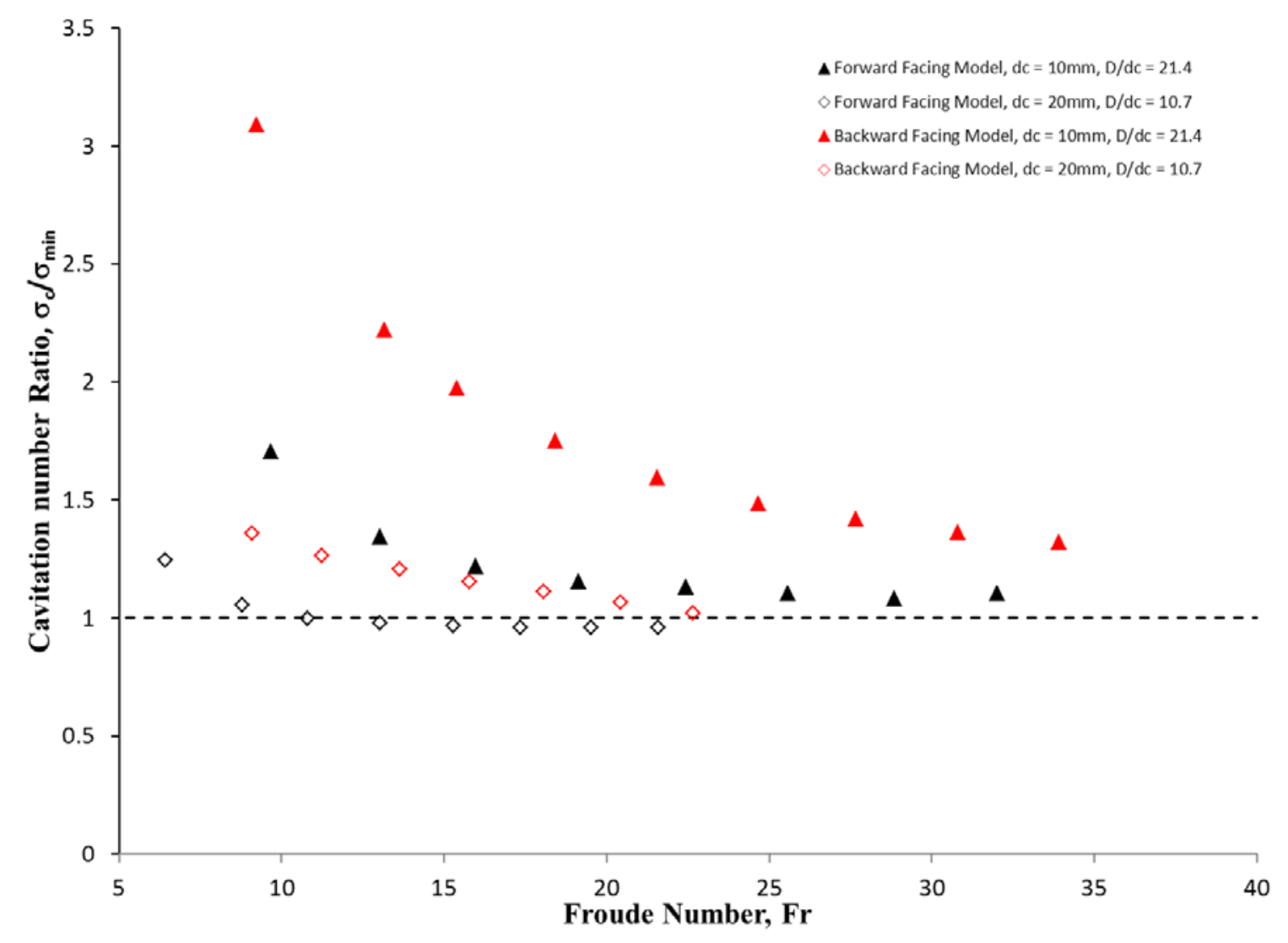

Figure 3.2.5: Ratio of cavitation numbers, $\sigma_{\mathrm{c}} / \sigma_{\min }$, plotted against Froude number. Backward facing model data is shown in red, while forward facing model data is shown in black. Data are for disk cavitator sizes of 10 and $20 \mathrm{~mm}$.

A significant difference between the two models is that the backward facing model's transition from a foamy to a clear supercavity was observed at a lower ventilation rate than for the forward facing model for similar conditions. The presence of a body inside the supercavity resulted in an increase in the required ventilation rate at which the cavity transitioned from a short foamy cavity to a long, clear cavity. One possible explanation for this is that the absence of a body inside the supercavity produces a different internal flow pattern where there is more recirculation which could lead to 
transition at a lower value for the entrainment coefficient. This hypothesis could be explored further in the future.

Qualitatively, the results found at SAFL agreed with the results form Swanson and O’Neill (1951) in the sense that once the twin-vortex regime was established, there was little variation in cavitation number with a significant increase in ventilation rate. A minimum entrainment value, the transition value from re-entrant jet to twin vortex, seen in Figure 3.2.1 was not observed in the experiments at SAFL. The results at SAFL agreed with the numerical predictions from Spurk (2001) qualitatively in the sense that as the cavitation number decreased to its minimum value, the entrainment coefficient increased gradually. There is a significant quantitative difference in comparison to the numerical predictions due to the presence of blockage. For the backward facing model, there is a gap in the curve associated with the transition from re-entrant jet to twin vortex. There is a drastic decrease in cavitation number once the transition point has been exceeded. The reliance on processing of images to find the cavitation number in the reentrant jet region makes it difficult to further explore the differences between SAFL experiments and the predictions from Spurk (2001) and the results from Swanson and O’Neill (1951).

A distinction should be made between two ‘types' of re-entrant jet. The first type is the short, foamy supercavity where the entire supercavity is foamy and is influenced by the re-entrant jet. The second type is observed during the transition to a long, clear supercavity or during the collapse back to a short, foamy supercavity. For the second type, a small re-entrant jet is observed at the closure of the supercavity, only affecting part of the surface of the supercavity. These differences can be observed in Figure 3.2.6. 
The first type of re-entrant jet supercavity was found to be stable but unsteady while the second was found to be unstable and unsteady, observed only during transition to and from the twin vortex regime for both models.
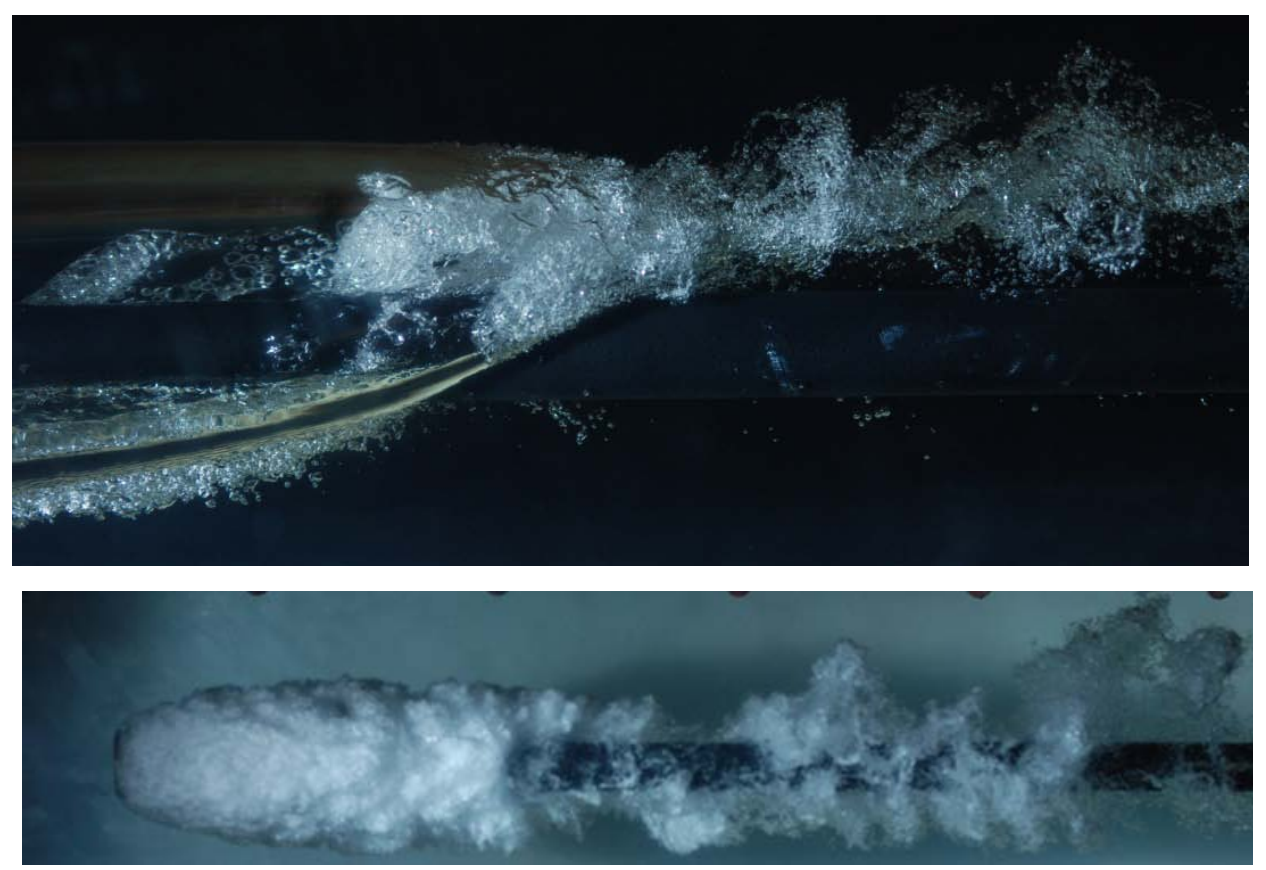

Figure 3.2.6: Comparison of the two types of re-entrant jet closure methods. Top: Reentrant jet observed during the transition from a long, clear supercavity to a short, foamy cavity. Bottom: Re-entrant jet closure observed before the transition to a long, clear supercavity.

In Figure 3.2.7 snapshots from a high speed video show the development from short, foamy cavity to a long, clear supercavity. The video is taken by suddenly increasing the ventilation rate from a value much less than the required value for transition to a value known to be above the critical transition value. As seen below, initially there is a short, foamy cavity where no part of the cavity is transparent. As time progresses and the supercavity grows in length. The cavity becomes clear and the area of the supercavity that is affected by a re-entrant jet decreases. A transition from the first type of re-entrant jet to the second type is observed. As the supercavity grows in length the small recirculation region at the closure of the supercavity disappears until it is finally replaced by the twin vortex method of cavity closure, where no water is splashed back 
into the supercavity. This behavior was observed for all model configurations tested, though only the backward facing model with a $10 \mathrm{~mm}$ cavitator is shown.

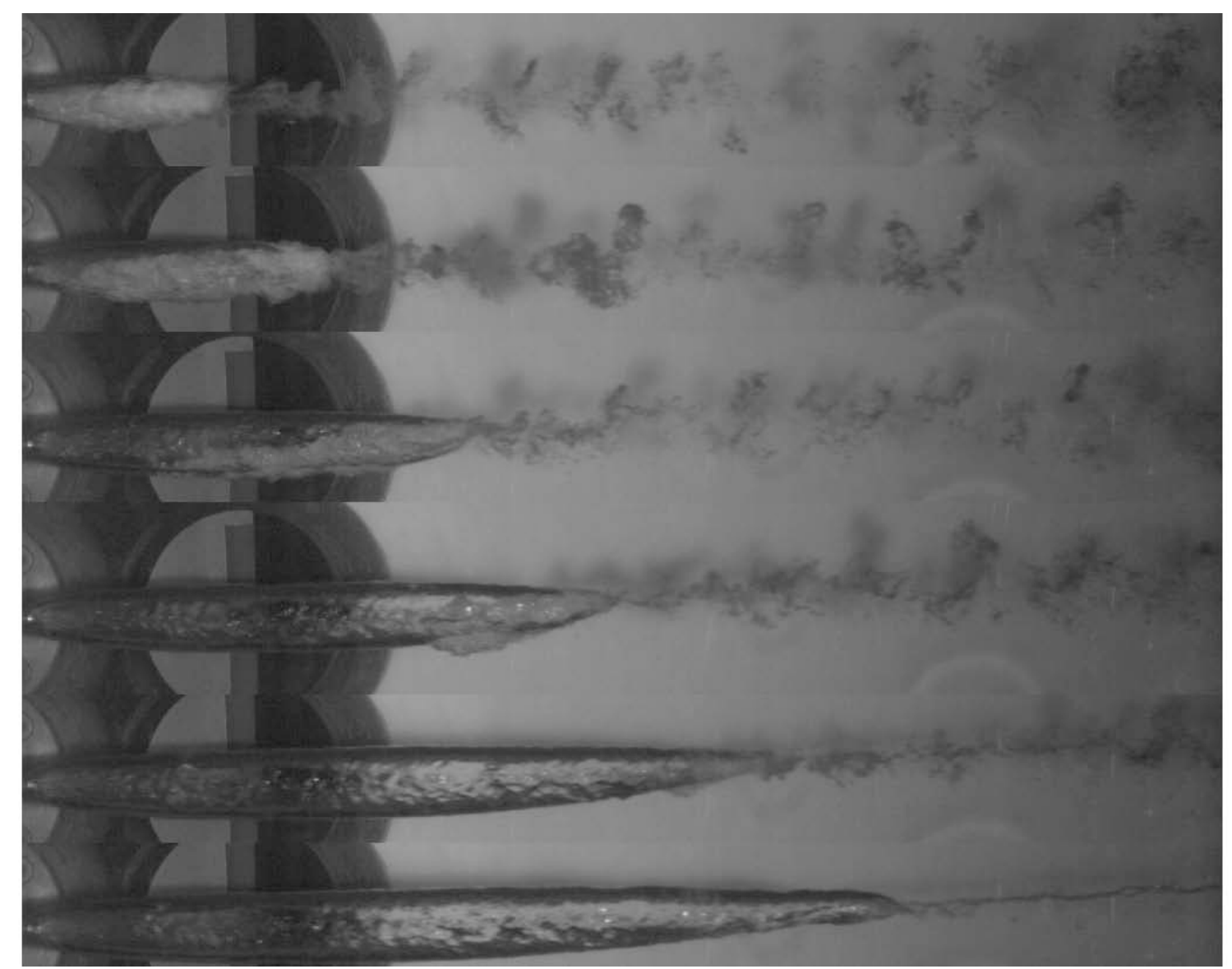

Figure 3.2.7: Supercavity development. Backward facing model, dc $=10 \mathrm{~mm}, \mathrm{Fr}=25.5$. Time progresses from top to bottom. 


\subsection{Froude Number Effects}

For artificial supercavitation, when the underwater vehicle is initially launched, supercavitation is achieved at Froude number values much less than that required for natural supercavitation and buoyancy effects must be taken into account. For natural supercavitation, the supercavity is axisymmetric and Froude number effects are negligible due to the high speed of the vehicle. It has been observed that there are significant Froude number effects on the relationship between the entrainment coefficient and cavitation number. In general, a change in Froude number results in a horizontal shift of the curve for air entrainment coefficient plotted against artificial cavitation number, having no significant effect on the shape of the curve. An increase in Froude number leads to a negative shift of the entire curve. The two ways to vary the Froude number are to increase, or decrease, the velocity or change the characteristic length, which for current research is the diameter of the cavitator. May (1975) provided a plot with a summary of the results from Campbell-Hilborne (1958), Swanson-O'Neill (1951) and Cox-Clayden (1956) for $1 \frac{12}{2}, 3 / 4$, and 1 inch diameter discs. This is shown below.

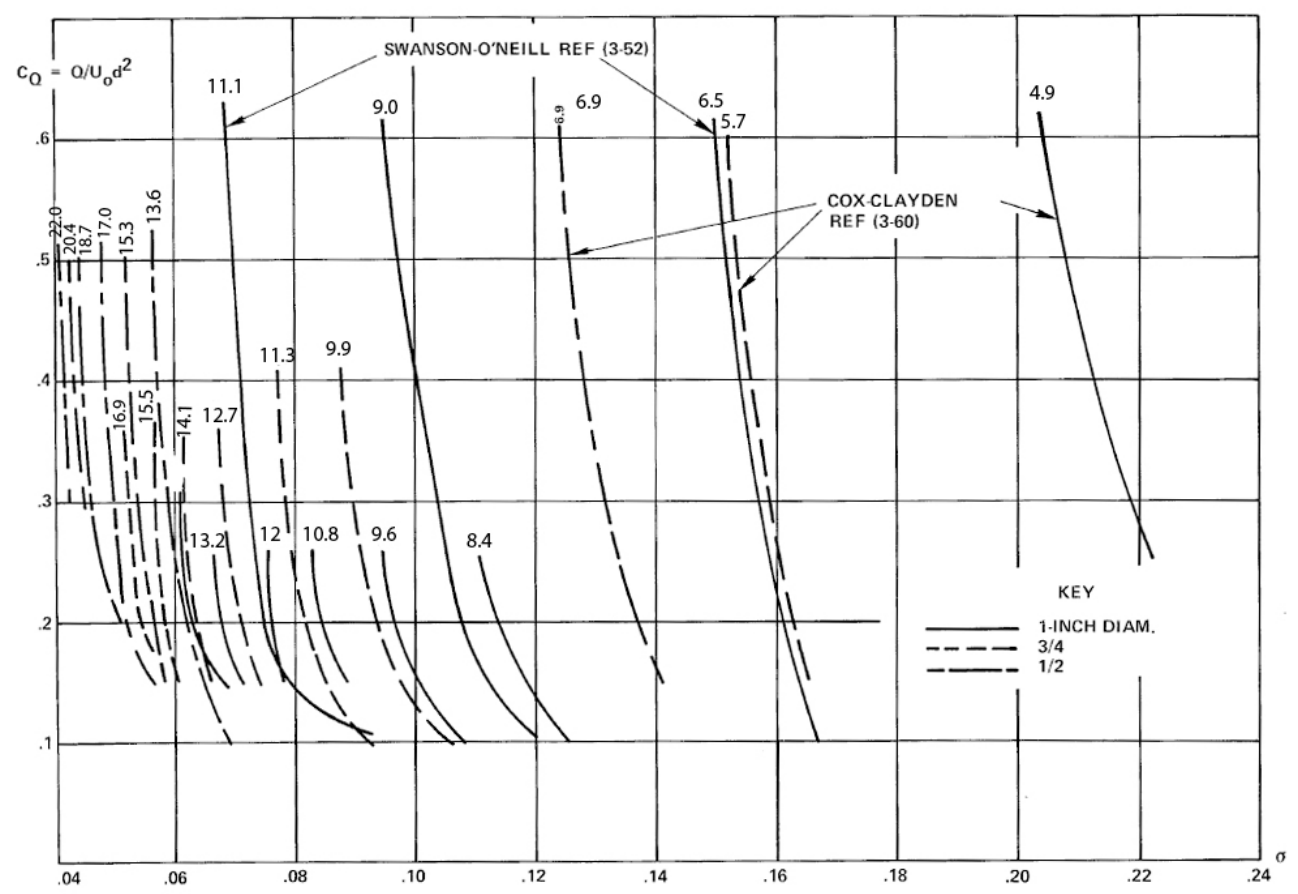

Figure 3.3.1: Ventilation curves for supercavities due to small disks. Data from Campbell-Hilborne (1958) except where labeled. Froude numbers are indicated above curves. (from May, 1975) 
In Figure 3.3.2 data obtained from the $10 \mathrm{~mm}$ and $20 \mathrm{~mm}$ cavitators for both models are compared to the $1 / 2$ inch and $3 / 4$ inch disc results presented in May's plot. For these experiments, the Froude number was held constant while the ventilation rate was increased, then decreased. As mentioned earlier, once a clear supercavity was established the cavitation number varied little with a significant increase in ventilation. Results correspond to long, clear supercavities only, where the cavitation number is determined from direct measurement of cavity pressure.

For both models, as the Froude number increased, the cavitation number associated with a clear supercavity decreased. A significant difference between the two models is that higher Froude numbers were required for the backward facing model to reach similar cavitation numbers seen by the forward facing model for the same size cavitator. Given that the freestream velocity for a given Froude number is the same for a given cavitator, one possible explanation is that the absence of a body inside the supercavity serves to increase the pressure difference between the freestream pressure upstream and the pressure inside the supercavity. Another possible explanation is that the upstream hydrofoil which the cavitator is mounted behind is affecting the flow physics, influencing the cavitation number. This will be discussed later.

Another significant difference is the gap in cavitation number values between the $10 \mathrm{~mm}$ and $20 \mathrm{~mm}$ cavitator values over a similar range of Froude numbers for both models. This difference is attributed to blockage limiting the minimum attainable cavitation number for a given diameter cavitator. As mentioned earlier, Brennen (1969) and Tulin (1961) each carried out numerical simulations to determine the minimum cavitation number for a given blockage ratio. For the higher Froude numbers tested for each model, the cavitation number agreed well with the minimum values predicted by Brennen and Tulin. The significant difference between the data collected at SAFL and that of Campbell and Hilborne (1958) can also be attributed to blockage. Campbell and Hilborne's experiments were carried out in the absence of blockage, allowing them to reach much lower cavitation numbers than those attainable in the SAFL water tunnel. It should be noted that minimum cavitation number dictated by blockage is not seen at every Froude number for a given cavitator, but only for the higher values. Just as with 
the case of no blockage, where a cavitation number of zero is the limit at infinite Froude number, the cavitation number is greater than its minimum value where Froude number effects are significant. As the Froude number approaches infinite, where gravitational effects are negligible and an axisymmetric cavity is present, the cavitation number approaches its minimum value.

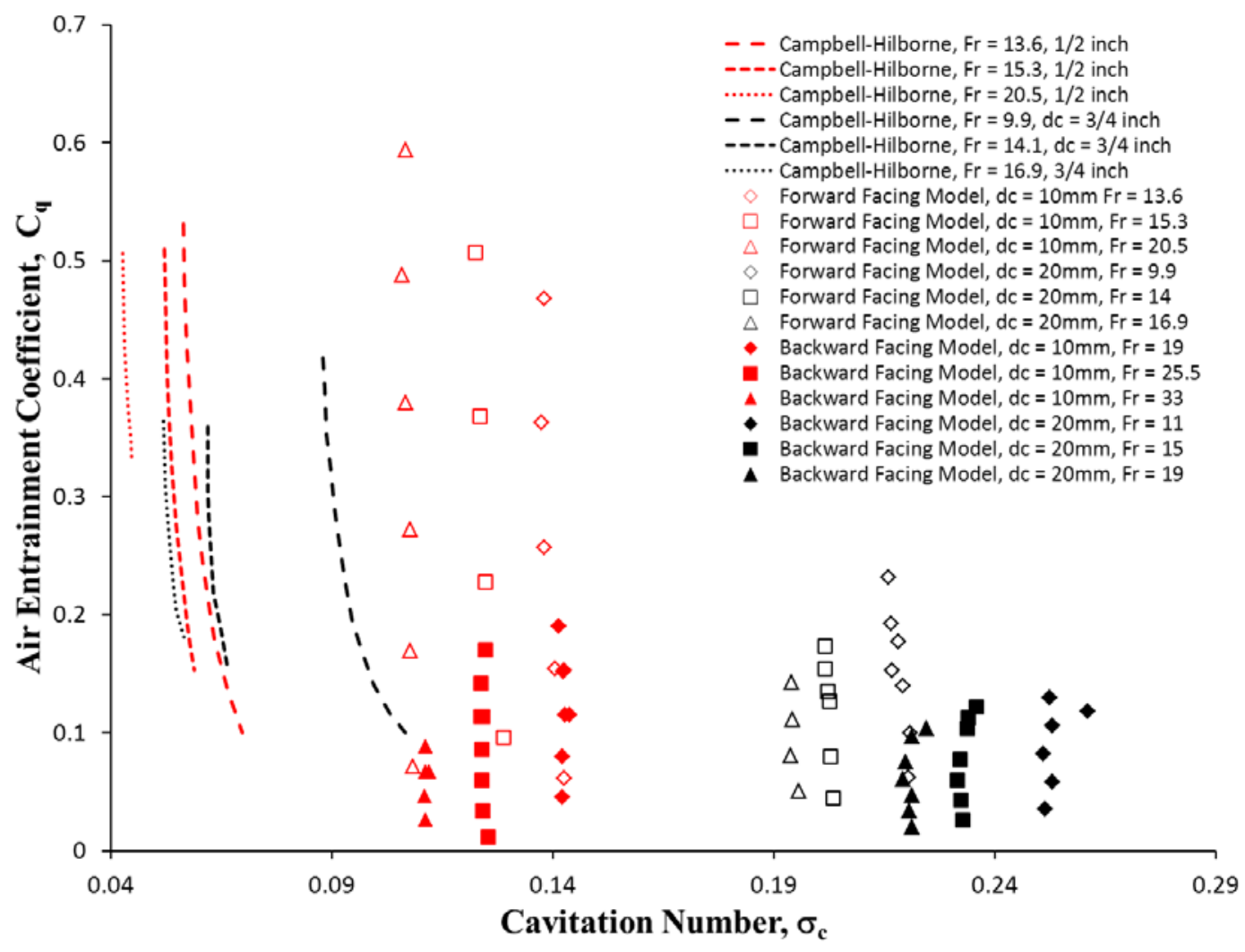

Figure 3.3.2: Entrainment Coefficient plotted against cavitation number for constant Froude number. Comparison between the forward and backward facing models with results from Campbell-Hilborne (1958) as presented by May (1975).

Given that the cavitation number did not vary significantly with ventilation rate once a clear supercavity was established, the next step was to see how the cavitation number varied with Froude number as the entrainment coefficient was held constant. This would provide more information on how the cavitation number varied over a larger range of Froude numbers. The results for the forward facing model at the 6 cavitator sizes tested can be observed in Figure 3.3.3. For each cavitator tested, a clear cavity was formed and then the ventilation rate was adjusted such that the entrainment coefficient 
was held constant. It was not uncommon for the ventilation rate needed to maintain a constant entrainment coefficient to be less than the transition value necessary to go from a short, foamy supercavity to a long, clear supercavity. As discussed earlier, once a clear supercavity was formed, the ventilation rate could be decreased below the transition value without a collapse of supercavity due to some hysteresis effect. This allowed for the entrainment rate to be held constant over the range of Froude numbers tested, even if it was less than the transition value. At lower Froude numbers, associated with velocities less than $5 \mathrm{~m} / \mathrm{s}$, the cavitation numbers observed were greater than the minimum predicted by Brennen (refer to Table 3.1.1 values). This behavior was observed for each cavitator size tested. As the Froude number increased, the cavitation number gradually approached the minimum value dictated by blockage. Once the minimum value was reached, an increase in Froude number had a negligible effect on the cavitation number.

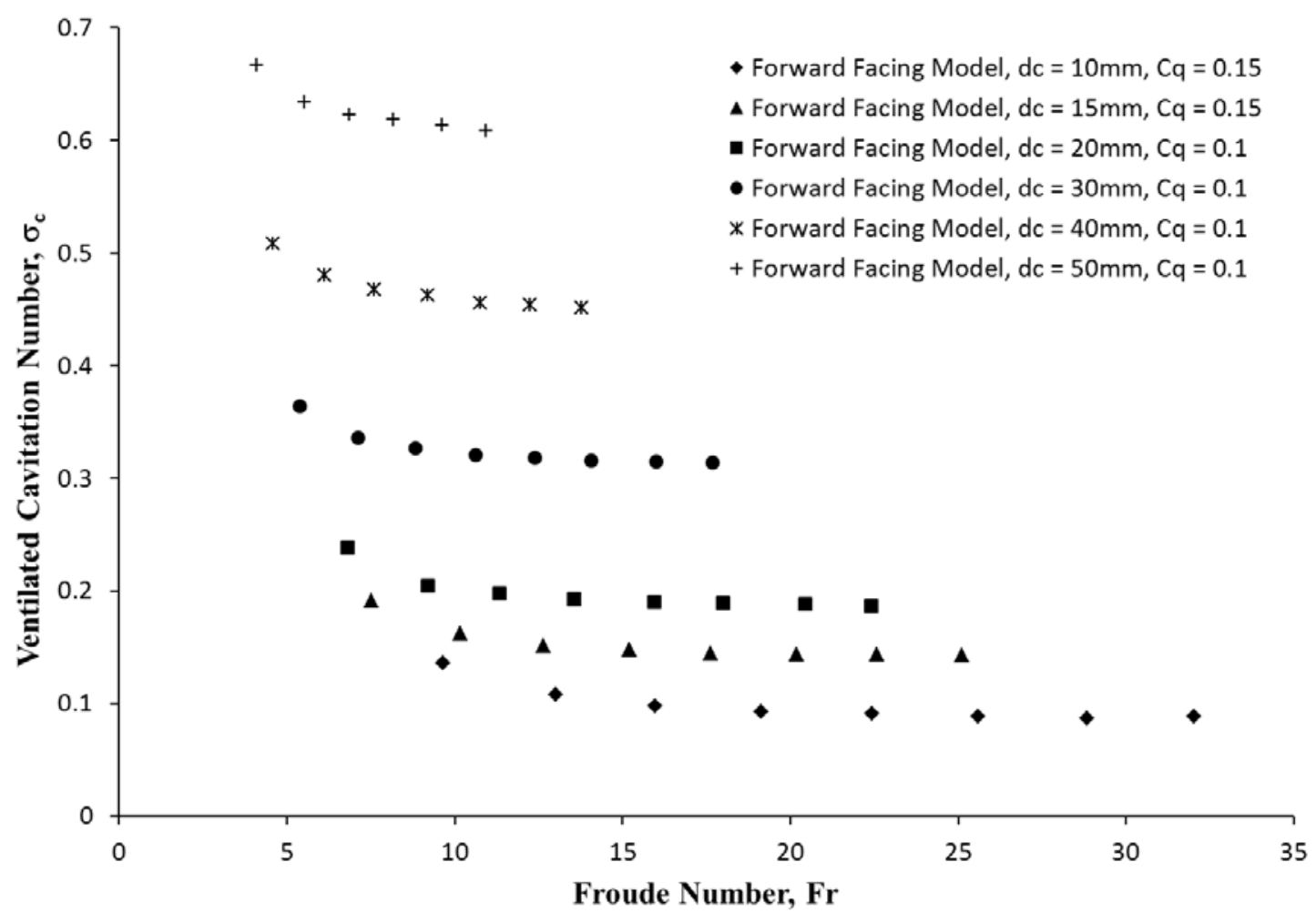

Figure 3.3.3: Cavitation number plotted against Froude number for constant entrainment coefficient, $\mathrm{C}_{\mathrm{q}}=$ constant, for various disk cavitators (Forward facing model results only shown above). 
The backward facing model yielded results very different than the forward facing model. A comparison between both models for cavitators of 10 and $20 \mathrm{~mm}$ can be seen in Figure 3.3.4. As the Froude number varied for the backward facing model, the cavitation number continually decreased until it reached the minimum value dictated by blockage. The backward facing model took a significantly higher Froude number to reach the minimum cavitation number than the forward facing model. A possible explanation for this will be explained further later.

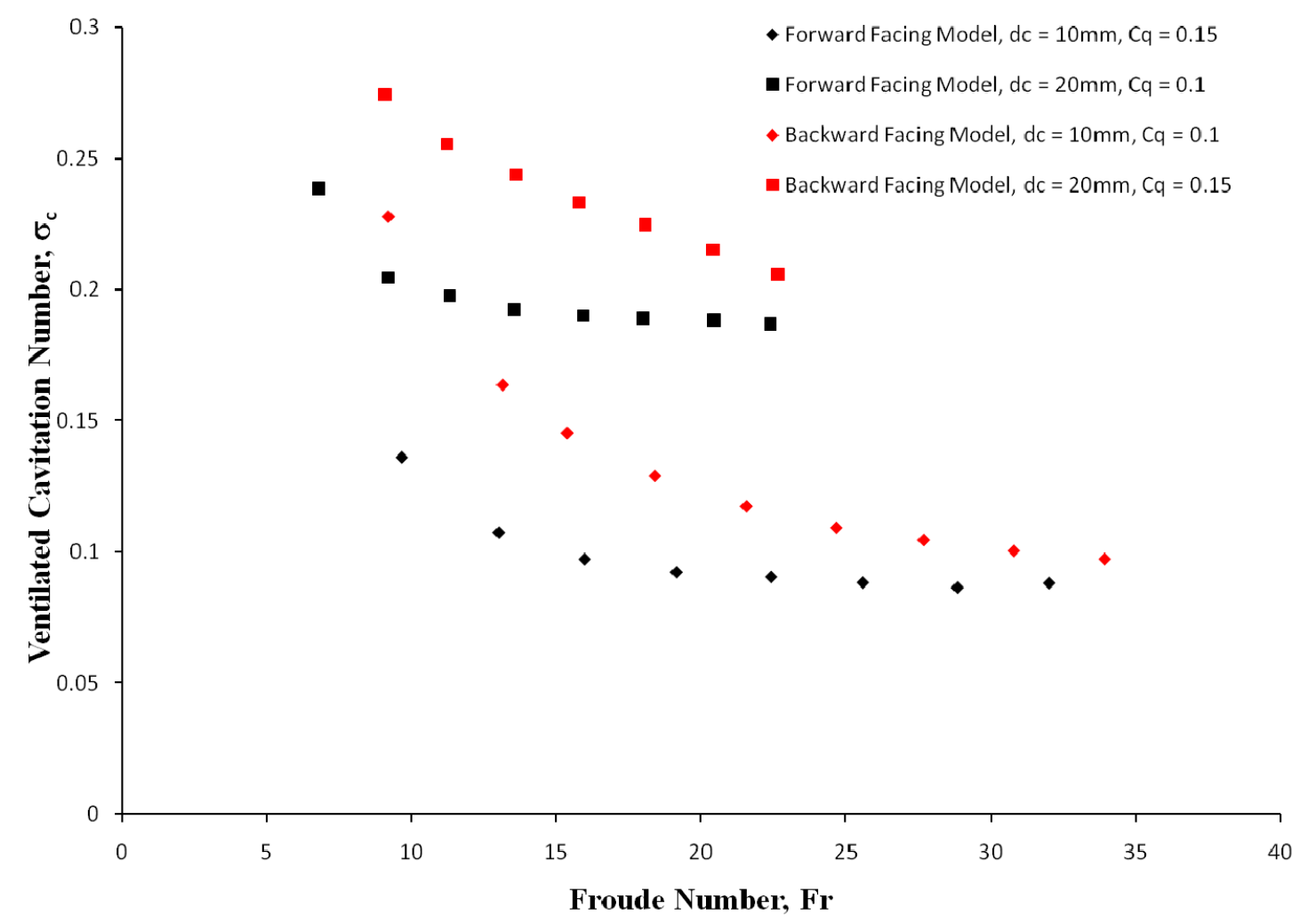

Figure 3.3.4: Comparison of cavitation numbers at constant entrainment coefficient, $\mathrm{C}_{\mathrm{q}}=$ constant, for the 10 and $20 \mathrm{~mm}$ disk cavitators with the backward and forward facing models.

It is interesting to note that for the forward facing model, the supercavity is visibly still affected by buoyancy at Froude numbers where the cavitation number has already reached its minimum. This can be observed in Figure 3.3.5, where a progression of images for the forward facing model with a $10 \mathrm{~mm}$ cavitator are shown overlaid on the plot of cavitation number against Froude number. The assumption of an infinite Froude 
number in Brennen and Tulin's calculations implies an axisymmetric supercavity. At finite Froude numbers, buoyancy effects can be seen by a pitching up of the supercavity downstream of the cavitator. For lower Froude numbers, the pitching up is much more prevalent than at higher Froude numbers. As the Froude number increases, by an increase in velocity, the supercavity behaves more like an axisymmetric cavity. The easiest way to see this is to observe the location of the closure of the supercavity. The supercavity closes above the centerline of the supercavity when buoyancy effects are significant. Similar results were seen for all cavitators tested with the forward facing model, though only the results for a $10 \mathrm{~mm}$ cavitator are shown below. These observations suggest that an infinite Froude number is not necessary to utilize results from Brennen's simulation for the forward facing model, a result discussed earlier as well.

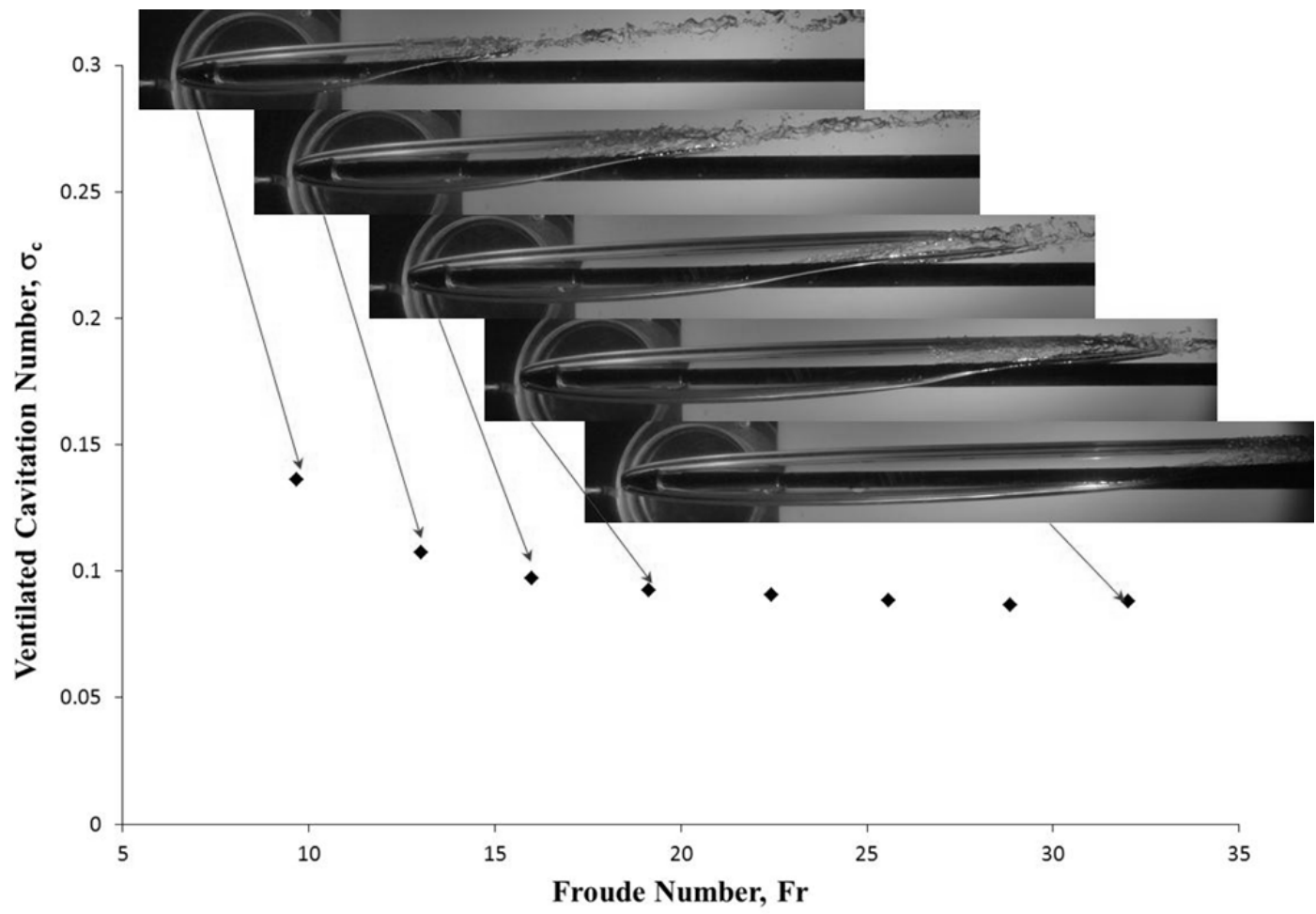

Figure 3.3.5: Ventilated cavitation number plotted against Froude number for a $10 \mathrm{~mm}$ cavitator with the forward facing model. Images show how the effects of buoyancy decrease with an increase in Froude number. 
For the backward facing model, slightly different results were observed. Just as with the forward facing model, the effects of buoyancy decreased with an increase in Froude number. As the Froude number increased, the supercavity became more axisymmetric and the cavitation number approached the minimum value predicted by Brennen. As seen earlier, the rate at which the cavitation number approached the minimum value predicted with an increase in Froude number was much slower with the backward facing model. Once the supercavity was very close to axisymmetric, the agreement with Brennen was very good. At lower Froude numbers, where buoyancy effects are significant, the agreement with Brennen is poor. A possible reason for the difference between the two models will be discussed later. For the backward facing model, an axisymmetric supercavity was necessary for good agreement with Brennen's numerical model. In Figure 3.3.6 the progression of images is overlaid on the plot of measured cavitation number against Froude number for the $10 \mathrm{~mm}$ cavitator. Similar results were observed with the $20 \mathrm{~mm}$ cavitator as well.

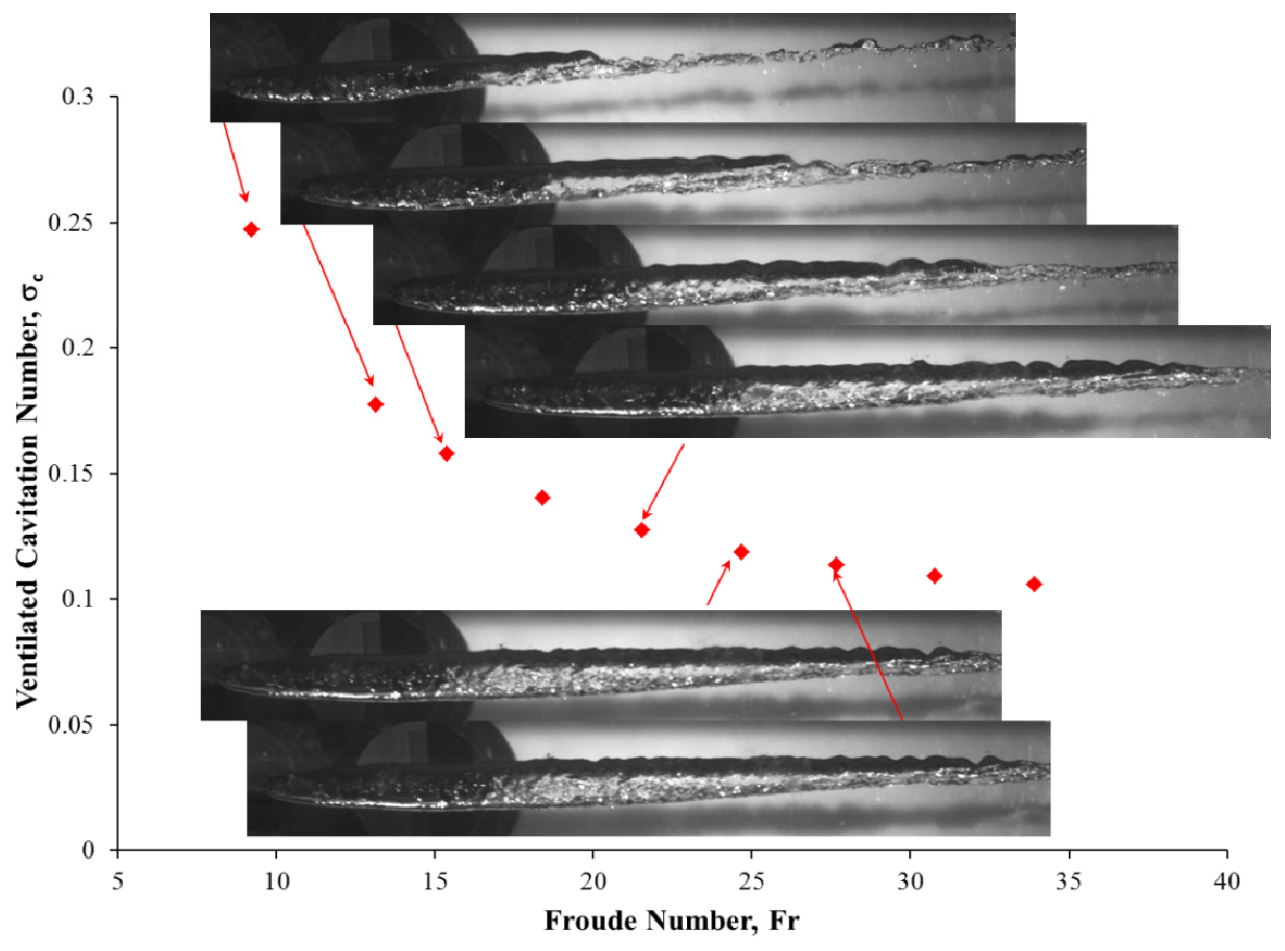

Figure 3.3.6: Ventilated cavitation number plotted against Froude number for a $10 \mathrm{~mm}$ cavitator with the backward facing model. Images show how the effects of buoyancy decrease with an increase in Froude number. 


\subsection{Unbounded Flow Equivalence}

A natural question in the study of supercavitation is the correspondence of the results in water tunnels to unbounded flow. It has been shown that for two-dimensional flows the presence of walls does not change the variation of the drag coefficient with cavitation number when compared to unbounded flow (Karlikov-Sholomovich 1966). It is assumed that this holds true for axisymmetric flows as well. This relation is expressed as

$$
C_{D}=c_{x_{o}}(1+\sigma)
$$

This reduces the effect of the presence of walls to a correlation between the cavitation numbers in bounded and unbounded flows. Several models have been proposed to correct the water-tunnel cavitation number to a freestream value.

Karlikov and Sholomovich (1966) proposed a theoretical model that applies to axisymmetric flows (applicable to the results obtained at SAFL) as well as twodimensional flows. The unbounded cavitation number was expressed as

$$
\begin{gathered}
\sigma_{\infty}\left(\sqrt{1+\sigma-\sigma_{\infty}}-1\right)(\sqrt{1+\sigma}-1)\left(1+\sigma_{\min }\right)= \\
=\left(\sqrt{1+\sigma_{\min }}-1\right)^{2}\left[\sigma_{\infty}(1+\sigma)-\sqrt{1+\sigma}\left(1+\sigma_{\infty}\right)\left(\sqrt{1+\sigma}-\sqrt{\left.1+\sigma-\sigma_{\infty}\right)}\right]\right.
\end{gathered}
$$

For most cavitation experiments, the values for $\sigma$ and $\sigma_{\infty}$ are small enough that series expansion can be used to simplify the long equation above to

$$
\sigma_{\infty}=\frac{2 \sigma_{c}^{2}-\sigma_{\min }^{2}}{2 \sigma_{c}}
$$

where $\sigma_{\min }$ corresponds to the minimum attainable cavitation number as dictated by flow choking (results from Tulin (1961) and Brennen (1969) can be used for $\sigma_{\min }$ ). It should also be noted that Karlikov and Sholomovich also made the assumption of an axisymmetric supercavity, or once again an infinite Froude number assumption.

Garabedian (1956) carried out calculations for the dimensions of an axisymmetric supercavity. He assumed a steady, axially, symmetric, irrotational and incompressible liquid with zero blockage for derivation of his theory. Another key assumption in the development of his theory was that the supercavity followed a Riabouchinsky model, 
which assumes that the supercavity has a symmetrically shaped nose and tail. One of the equations derived by Garabedian for the dimensions of the supercavity was

$$
D_{c}=d_{c} \sqrt{\frac{c_{x_{0}}(1+\sigma)}{\sigma}}
$$

where $\mathrm{D}_{\mathrm{c}}$ is the maximum diameter of the cavity, $\mathrm{c}_{\mathrm{xo}}$ is the drag coefficient at a cavitation number of zero, and $\sigma$ is the cavitation number. For a disk, $\mathrm{c}_{\mathrm{xo}}$ is equal to 0.827. For Karlikov and Sholomovich (1969), once their ventilated cavitation numbers were correlated to open water flows, their experimental data for various blockage ratios collapsed to a curve, which plotted the ratio of maximum cavity diameter to cavitator diameter as predicted by the equation above.

The results from the forward facing model conducted at SAFL are summarized in Figure 3.4.1. Once again, images were processed using the Matlab code generated by Dr. Hambleton. The values for the cavitation number were determined by direct measurement of the supercavity pressure. These results are shown by black symbols. Garabedian's equation is shown by the black line in the plot. Initially, the collected data do not show good agreement with Garabedian. This was due to the effects of blockage, as previously discussed. The Karlikov and Sholomovich equation was applied to the data to find the equivalent freestream cavitation number values. For the 10, 15, and 20mm cavitators, the compact form of the equation was used. For the 30, 40, and 50mm cavitators, the values for the measured cavitation number, and the minimum values dictated by blockage, were large which required the extended form to be used. The equivalent freestream cavitation number values are shown in red in Figure 3.4.1. After Karlikov and Sholomovich's equation was applied to the data obtained at SAFL, the results showed good agreement with the curve generated by Garabedian's equation. The 10,15 , and $20 \mathrm{~mm}$ cavitators showed the most agreement with the curve while the 30,40 , and 50mm cavitators showed some deviation from the curve over the range of cavitation numbers presented. A possible explanation for this is that the walls of the test section have a much more significant effect on the shape of the cavity, as well as the flow dynamics for the larger cavitators. These results show support for the use of Karlikov 
and Sholomovich's equation to find equivalent freestream cavitation numbers for values obtained during water tunnel testing up to a certain blockage ratio.

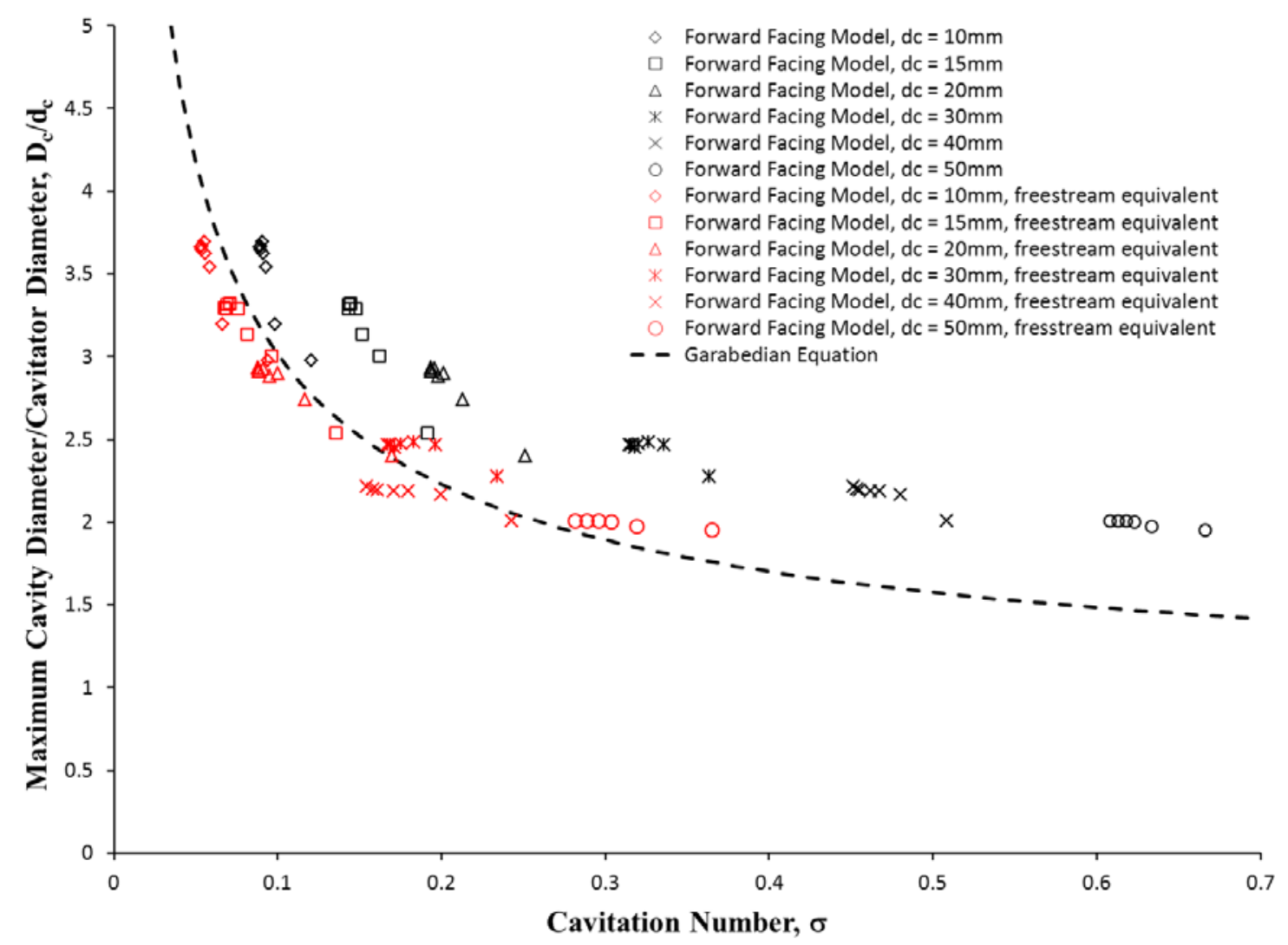

Figure 3.4.1: Comparison of experimental data to Garabedian's predictions for supercavity shape. Experimental cavitation numbers from the SAFL water tunnel from are shown in black. The equivalent unbounded cavitation numbers, as computed by

Karlikov and Sholomovich's equation are shown in red.

For freestream flows, independent of the effects of blockage, the variation of cavitation number and Froude number should be the same for any size disc cavitator. To investigate this, a comparison was made for the 6 cavitator sizes tested with the forward facing model. Ideally, each data set would collapse to the same curve when plotting cavitation number against Froude number. Figure 3.4.2 illustrates the plot of equivalent freestream cavitation number against Froude number for the results collected at SAFL. It was found that for the 10,15 , and $20 \mathrm{~mm}$ cavitators, agreement with results presented by May (1975) was good. For the larger cavitators, 30, 40, and 50mm, agreement was poor. It is interesting to note that while the 30 and $40 \mathrm{~mm}$ cavitators did not show agreement 
with the smaller cavitators, they showed good agreement with each other. At this point, little is known as to why this is the case. One theory is that the size of the cavities created by the larger cavitators is so large that viscous effects from the walls are influencing the flow. Another theory is that for the larger size cavitators, greater than $20 \mathrm{~mm}$, the non-axisymmetry of the supercavity is such that Karlikov and Sholomovich's equation cannot be applied. Up to cavitator sizes of $20 \mathrm{~mm}$, a blockage ration of 10.2 , the assumption of infinite Froude number, or an axisymmetric supercavity, is not critical above certain finite Froude numbers. For cavitators greater than $30 \mathrm{~mm}$ in diameter, this assumption is critical for all finite Froude numbers.

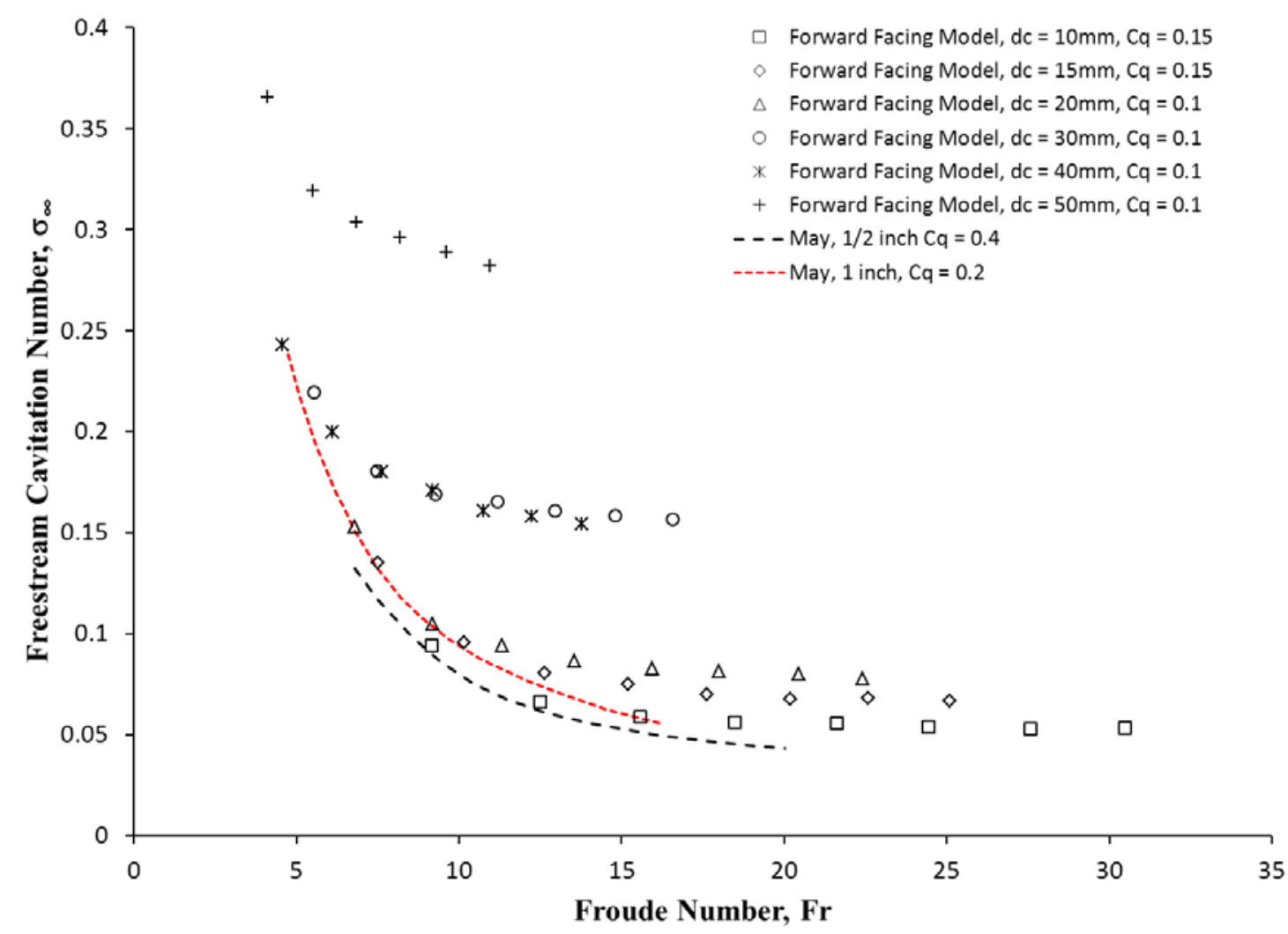

Figure 3.4.2: Freestream cavitation number plotted against Froude number. Forward facing model for all the cavitator sizes tested. 


\subsection{Cavity Closure Mechanisms}

A detailed examination of the supercavity closure, an essential element in the physics of the ventilation demand, was done. The product $\sigma_{\mathrm{c}} \mathrm{Fr}$ was always greater than one for the experiments conducted on both models, which Campbell (1958) predicted for the re-entrant jet regime. The product $\sigma_{\mathrm{c}}^{3 / 2} \mathrm{Fr}^{2}$ was also found to be greater than 1.5 and often greater than 10, which Buyvol (1980) attributed to the re-entrant jet regime. This was rarely the case, as the twin-vortex entrainment mechanism was the dominant method observed. Even after equivalent freestream cavitation number values were found, both products still predicted the re-entrant jet to be the entrainment mechanism. The re-entrant jet method of cavity closure was found to be unstable and was only observed in the transition from a short, foamy supercavity to a long, clear supercavity, or in the collapse from a long clear supercavity to a short, foamy supercavity. Once again it should be highlighted, that a distinction is made between a clear supercavity with a small re-entrant jet region at the tail of a supercavity, and a foamy cavity which is completely opaque (Figure 3.2.6).

An unexpected observation found that while the body of the supercavity was stable (a constant length and diameter), the vortex pair at the closure of the supercavity was not. At one instant a vortex core was present, a few moments later, the vortex core would collapse followed by a reformation of a vortex core. This was an ongoing process for a give set of conditions and was seen for both models. This phenomenon is seen below in Figure 3.5.1. 

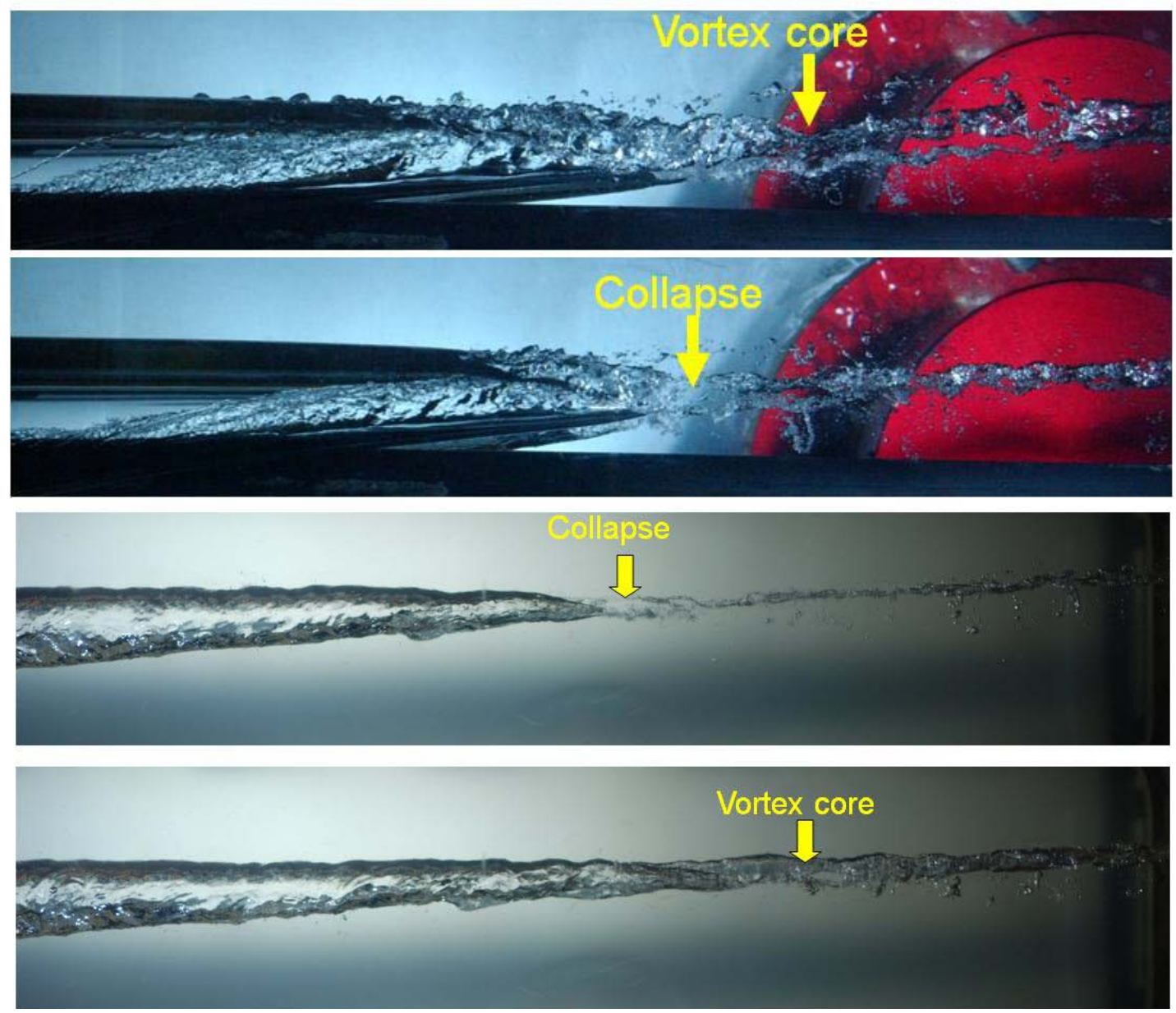

Figure 3.5.1: Examples of twin-vortex closure for both models. Top two images: forward facing model. Bottom two images: backward facing model.

At low Froude numbers and small ventilation rates, a third mode of air entrainment was observed for both models. For these conditions, two sets of vortex pairs were observed, one pair above the other. Just as the case with the single pair of vortices, an ongoing process of vortex core formation and collapse was seen. An interesting observation was that the two pairs of vortex cores did not collapse simultaneously, but at different times. For all the experiments conducted at SAFL the cavitator was held at zero degrees angle of attack, but the conditions present for the 'quad' vortex method of cavity closure did not match those predicted by Kapankin (1984). The reason for the two sets of vortex pairs is not fully understood at present. 

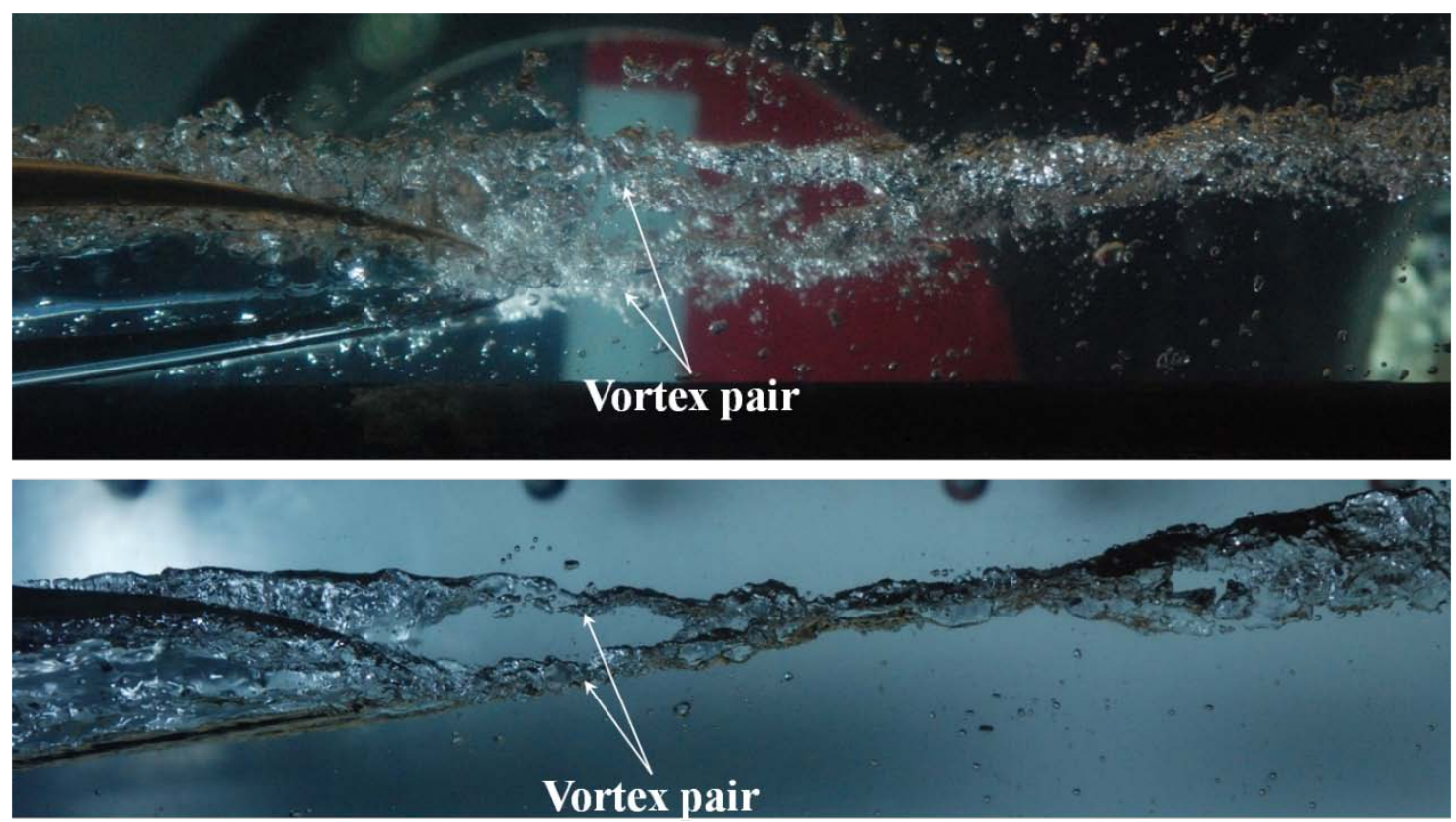

Figure 3.5.2: Example of quad-vortex closure. Top: forward facing model. Bottom: backward facing model. Note that the images are side views of the supercavity. Each vortex seen in the images (top and bottom) has a matching vortex behind it.

\subsection{Model Comparison}

The significant difference in cavitation numbers observed between the forward and backward facing models is due to the presence of the hydrofoil mounting strut upstream of the cavitator. For the forward facing model, the flow upstream of the model is undisturbed. The only pressure reduction is due to the cavitator. This is not the case with the backward facing model. For the backward facing model, the presence of a hydrofoil upstream introduces a pressure reduction due to drag. The wall supports for the hydrofoil also have an effect on the pressure reduction due to drag. A simple momentum balance can be done to check this. For the analysis, the hydrofoil, a NACA 16-015 profile, is placed in a tunnel with a diameter equal to equivalent diameter of the SAFL test section. For simplicity, it is assumed that there is complete velocity recovery (i.e. velocity before and after the hydrofoil are the same). The control volume for the analysis can be seen below in Figure 3.6.1. 


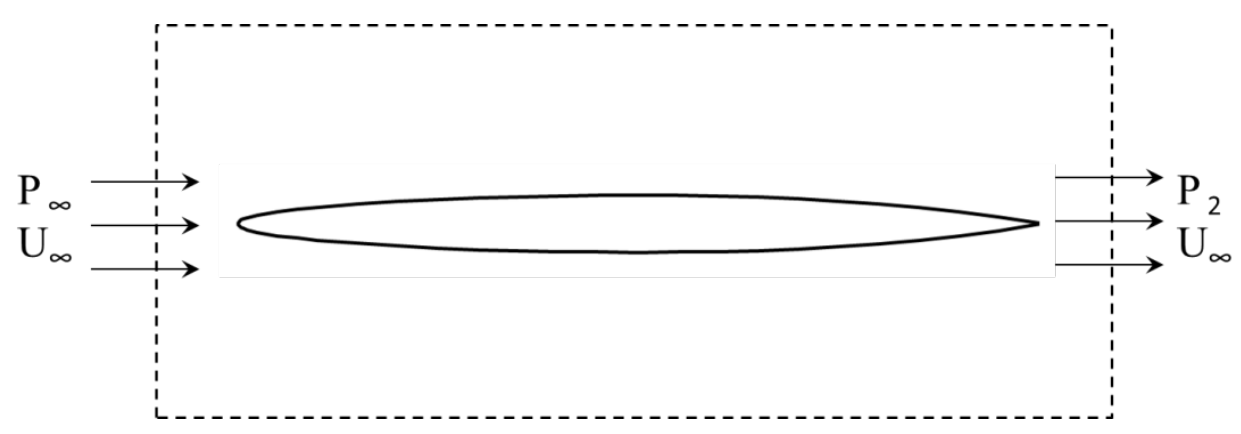

Figure 3.6.1: Control volume for analysis of hydrofoil drag.

Evaluating the conservation of momentum for the control volume, the following relationship is found

$$
\left(P_{\infty}-P_{2}\right) A_{0}=\frac{1}{2} \rho U_{\infty}^{2} C_{D} S
$$

where $A_{0}$ is the cross section of the test section, $S$ is the hydrofoil wing area (span*chord), and $\mathrm{C}_{\mathrm{D}}$ is the drag coefficient, which is unknown. After rearranging the equation,

$$
\frac{P_{\infty}-P_{2}}{\frac{1}{2} \rho U_{\infty}^{2}}=\left(\frac{S}{A_{0}}\right) C_{D}
$$

which can be directly compared to the difference in cavitation numbers for the same conditions between the two models. This determined if the computed drag coefficient was realistic. For lower Froude numbers, less than 15, for both size cavitators, a difference in cavitation number between models of 0.07 was not uncommon. This would mean that the drag coefficient was approximately 0.31. Given the various factors influencing the flow upstream of the cavitator, this is not an unreasonable estimate. For higher Froude numbers, also higher Reynolds numbers, there was less difference between the two models. Values of 0.03 were on the high end, which would yield a drag coefficient of 0.13 , also not an unrealistic value. It should be noted the previous analysis is an estimate and further experiments would need to be conducted to quantify more accurate values. 


\section{CONCLUSION}

\subsection{Conclusions}

The current study examined various aspects of a ventilated, supercavitating vehicle. Among these aspects was an extensive investigation of blockage effects that arise as a result of conducting experiments in a water tunnel Model configuration effects were also investigated. It was found that the model configuration had a significant influence on the cavitation number.

It was verified that the effect of blockage was to dictate a lower limit on the cavitation number. Comparison of experimental data, where the ventilated cavitation number was computed by direct measurement of cavity pressure, with numerical simulations from Brennen found that an infinite Froude number was not necessary to use Brennen's results to find the cavitation number. This is important for models where a means of directly measuring the cavity pressure is not possible. An interesting observation found that blockage shifted a family of curves for entrainment coefficient plotted against cavitation number.

As observed by various other researchers, variation of Froude number significantly affects the cavitation number observed once a clear supercavity has been established (for cavitation numbers greater than the blockage value). As Froude increased, the cavitation number associated with a clear supercavity approached the minimum value dictated by blockage. Once the minimum cavitation number associated with a given blockage ratio is reached, a further reduction in cavitation number by an increase in Froude number is not possible.

An unexpected hysteresis effect was observed. It was found that a stable supercavity could be sustained at ventilation rates less than that required to form the stable supercavity, with little to no effect on the supercavity dimensions and the cavitation number. This showed that the amount of gas needed to form a supercavity is far greater than the amount needed to sustain it.

The presence of the body inside the supercavity served to increase the required ventilation rate to create a stable supercavity, but did not seem to affect the method of gas removal. In contrast to the empirical observations of Campbell-Hilborne (1958), the 
product $\sigma_{\mathrm{c}} \mathrm{Fr}$ is consistently much greater than one even though the twin-vortex closure mechanism is dominant for stable supercavities for all configurations tested. While blockage can account for the differences seen in cavitation number, it does not account for the difference in closure mechanism observed.

Finally, a significant observation was that an infinite Froude number, which corresponded to an axisymmetric supercavity, was not necessary to utilize the results from Brennen and Tulin, up to a certain blockage ratio. A possible means of correlating water tunnel experiments to open water flows presented by Karlikov and Sholomovich (1966) was investigated. Their correlation shows promise for moderate blockage ratios. For the SAFL water tunnel, this corresponded to cavitator diameters up to $20 \mathrm{~mm}$. The reason why this correlation did not work for high blockage ratios was due to the fact that the assumption on an axisymmetric supercavity became critical.

The current research ties together research from various authors. The results from Brennen and Tulin are compared to experimental results which are then inserted into equations from Karlikov and Sholomovich and compared to Garabedian. A goal of the current research was to bridge the gap between Russian experiments conducted in the 1960's and 1970's and past and present American experiments. While, the current research does not completely accomplish this, it is a significant step in the right direction. 


\subsection{Recommendations for Future Work}

Further investigation of the backward facing model should be done to see if there is a correction factor that can be applied to account for the pressure drop associated with the upstream mounted hydrofoil. Once a correction factor is found, agreement between the two models should increase. This will provide insight into the effect of the absence of a body inside the supercavity. There are a few possible ways to evaluate this. One method could include the measurement of the pressure directly after the hydrofoil. A force balance could be used to find the drag on the model, which could prove advantageous not only for finding the pressure drop across the hydrofoil, but also to quantify the drag reduction advantages associated with artificial supercavitation.

For both models, the angle of attack of the cavitator should be investigated to see how that affects the cavitation number. This topic is of great interest when designing a controllable supercavitating vehicle. Changing the angle of attack of the cavitator adjusts the shape of the supercavity and the rate at which the surface pitches up, a characteristic of interest when trying to avoid planning effects (cavity surface-fluid interaction).

Further tests should be conducted to investigate the effects of the size of the body inside the supercavity. This could be done with the forward facing model by simply changing the diameter of the pipe between the mounting strut and the cavitator. A larger body inside the supercavity could decrease the amount of space available for the recirculation of gas inside the supercavity, causing a change in cavitation number. The presence of a larger body present inside the supercavity could also have an effect on the supercavity dimensions. When investigating the effects of the size of the body inside the supercavity, an important parameter may be the ratio between the cavitator diameter and the maximum diameter of the body.

Little is known about the flow inside the supercavity. Numerical simulations have been done in an attempt to visualize this, but little to no experimental data has been done. A smoke visualization, captured with a high speed camera, could possible provide insight into the internal flow of the supercavity. This would provide insight into how much air is being re-circulated back into the supercavity and how much is actually leaving via the hollow vortex tubes (for a twin-vortex supercavity). 
It is also desirable to observe supercavity characteristics in unsteady flow. The SAFL water tunnel is equipped with a gust generator with the ability to simulate unsteady flow over a range of velocities. The effects of the gust generator on the surface of the supercavity and the flow parameters could be investigated.

Schauer investigated void fraction and wake characteristics in a cavitating flow using Particle Image Velocimetry (PIV). In the bubbly wake, a technique was developed that used intensity gradients to compute the velocity field. Analyzing the grayscale levels of the images collected, Schauer calculated velocity and void fraction characteristics for the wake created by the re-entrant jet closure on his test body. There are several challenges that arise when using PIV in a cavitating wake. For bubbly flows, a method must be developed to distinguish individual bubbles from each other for correlation. This is extremely difficult when the void fraction of the flow is high and bubbles are clustered together. Schauer tried to address this challenge by using intensity gradients for correlation. A second problem arises when doing this. PIV is only a valid technique for finding the velocity field in the plane of the laser when the particles in that plane are being correlated. In high void fraction bubbly wakes, secondary Mie scattering from bubbles makes it impossible to distinguish which bubbles are in plane and which ones are not. The bubbles that are first contacted by a laser light sheet scatter the laser light causing illumination of out-of-plane bubbles. The out-of-plane bubbles can become even brighter than in-plane bubbles, making correlations based on intensity gradients inaccurate.

Recently, a velocity measurement technique has been developed by Innovative Scientific Solutions, Inc. This technique is referred to as Particle Shadow Velocimetry (PSV). Instead of using a laser, located perpendicular to the camera taking images, a light source is directly in-line with the camera. Using a very short depth-of-field and shadowing techniques, bubbles in the desired field-of-view can be distinguished from out-of-plane bubbles. This technique provides two advantages. This first is accurate tracking of in-plane bubbles, allowing for accurate determination of the velocity field in the bubbly wake. The second is the possibility for shadowgraphy, a technique used for determining the size and number of bubbles in a flow. Using PSV, the images lend 
themselves very nicely to analysis using shadowgraphy. This will provide insight into the void fraction of the wake of cavitating bodies and further insight into supercavity closure.

Currently, SAFL has all the necessary equipment and software to carry out PSV and shadowgraphy. SAFL also has high speed cameras that can be used to collect timeresolved data. A high-speed camera used for the PSV technique could provide insight into formation of a supercavity from its initial non-cavitating state, through the transition from re-entrant jet to the twin vortex regime. 


\section{BIBLIOGRAPHY}

Birkhoff, G., Zaramtonello, E., Jets, wakes and cavities. MIR, Moscow, Russia, 1964.

Brennen, C. A Numerical Solution of Axisymmetric Cavity Flows. Journal of Fluid Mechanics, vol. 37, part 4, pp. 671 - 688, printed in Great Britain, 1969.

Buyvol, V.N. Slender cavities in flows with perturbations (in Russian). Nauvoka Dunka Ed., Kiev (Ukraine), 1980.

Campbell, I.J., Hilborne, D.V. Air Entrainment Behind Artificially Inflated Cavities. Second Symposium on Cavitation on Naval Hydrodynamics, Washington DC, 1958.

Cohen H., Di Prima, R.C., Wall effects in cavitating flows. Sec. Symposium on Naval Hydrodynamics, August 25-29, 1958, ACR-38, 1960.

Cox, R.N., Clayden, W.A. Air entrainment at the rear of a steady cavity. Proceedings of the N.P.: Symposium on Cavitation in Hydrodynamics, London, 1956.

Epshtein, L.A., Characteristics of ventilated cavities and some scale effects. Proceedings of IUTAM Symposium on Rapid Non-Steady Liquid Flows, Leningrad, pp. 174-185, 1971.

Epshtein, L.A. Developed cavitation flows. Reprinted with translation from: additions by L.A. Epshtein in book J.S. Pearsall. "Cavitation” translated into Russian, publishing house MIR, Moscow, Russia, 1975. pp. 73-03. (Russ.)

Estevadeordal, J., Goss, L., PIV with LED: particle shadow velocimetry (PSV). $43^{\text {rd }}$ AIAA Aerospace Sciences Meeting and Exhibit, Meeting Papers, pp. 1235512364, 2005.

Fontecha, L.G. PIV Measurements in the Wake of a Supercavitating Body. M.S. Thesis, Chalmers University of Technology (carried out at the University of Minnesota), 2004.

Franc, J.P., Michel, J.M. Fundamentals of Cavitation, Springer, Netherlands, 2005

Garabedian, P.R. Cavities and Jets. Pacific Journal of Mathematics, 6, No. 4, pp. 611684, 1956.

Goss, L.P., Estevadeordal, J., Crafton, J.W., Velocity Measurements Near Walls, Cavities, and Model Surfaces Using Particle Shadow Velocimetry (PSV). Innovative Scientific Solutions, Inc., Dayton, O 
Kapankin, Y.N., Gusev, A.V. Experimental Research of Joint Influence of Fluid and Lift Power of Cavitator on Character of Flow in Cavity Rear Part and Gas Departure from it. Reprinted with translation from: Proceedings of CAHI 1984. -No.2244, Moscow, Russia, pp. 19-28 (Russ), 1984.

Karlikov, V.P., Sholomovich, G.I., Method of approximate account for the wall effect in cavitation flow around bodies in water tunnels. Izv. AN SSSR. Mekhanika Zhidkosti i Gaza, Vol. 1, No. 3, pp. 89-93, 1966.

Logvinovich G.V. Hydrodynamics of Free-Boundary Flows. Israel Program for Scientific Translations, Jerusalem, Israel (translated from Russian), 1972.

May, A. Water Entry and the Cavity - Running Behavior of Missiles.. Naval Surface Weapons Center, White Oak Laboratory, White Oak, Silver Spring, Maryland, 1975.

Paryshev, E.V., Theoretical study of the stability and pulsations of axisymmetric cavities. Tsagi 1907, pp. 17-40, 1978.

Peng, X., Wang, Z., Pan, S., Yan, K. Generation Mechanism of Ventilated Supercavitation in an Axisymmetric Body with Cavitator. Sixth International Symposium on Cavitation, CAV2006, Wageningen, The Netherlands, 2006.

Raffel, M. Particle Image Velocimetry. Springer, Heidelberg, New York, 2007.

Savchenko, Y.N. Supercavitation - Problems and Perspectives. RTO AVT Lecture Series on "Supercavitating Flows," Brussels, Belgium, 2001.

Semenenko, V.N. Artificial Supercavitation. Physics and Calculation. RTO AVT Lecture Series on "Supercavitating Flows,” Brussels, Belgium, 2001.

Schauer, T.J. An Experimental Study of a Ventilated Supercavitating Vehicle. M.S. Thesis, University of Minnesota, 2003.

Schiebe, F.R., Wetzel, J.M. Further Studies of Ventilated Cavities on Submerged Bodies. Office of Naval Research, Washington, D.C., 1964.

Song, C.S., Pulsation of ventilated cavities. J. Ship Res. 5(4), pp. 1-20, 1962.

Spurk, J.H. On the gas loss from ventilated supercavities. Acta Mechanica 155, 125-135, Springer-Verlag, 2002.

Stinebring, D.R., Billet, M.L., Lindau, J.W., Kunz, R.F. Developed Cavitation - Cavity Dynamics. RTO AVT Lecture Series on "Supercavitating Flows," Brussels, Belgium, 2001. 
Swanson, W.M., O’Neill, J.P. The Stability of an Air-Maintained Cavity behind a Stationary Object in Flowing Water. Hydrodynamics Laboratory, California Institute of Technology, Memorandum Report No. M-24.3, 1951.

Tulin, M.P. Lecture Notes on Supercavitating Flows. VKI Lecture Notes, University of California, Santa Barbara, 2001.

Tulin, M.P. Supercavitating Flows. In Streeter, V. (ed.), Handbook of Fluid Dynamics, McGraw-Hill, New York, pp. 1224-1246, 1961.

Waid, R.L. Cavity Shapes for Circular Disks at Angles of Attack. Hydrodynamics Laboratory, California Institute of Technology, Pasadena, California, Report No. E - 73.4, 1957.

Wosnik, M., Milosevic, I. Time - Resolved Particle Image Velocimetry (TR-PIV) in ventilated and naturally cavitating flows. $6^{\text {th }}$ International Symposium on Particle Image Velocimetry, Pasadena, California, 2005.

Wosnik, T., Schauer, T.J., Arndt, R.E.A. Experimental Study of a Ventilated Supercavitating Vehicle. Fifth International Symposium on Cavitation, CAV2003, Osaka, Japan, 2003.

Wosnik, T., Schauer, T.J., Arndt, R.E.A. Experimental Investigation of the Turbulent Bubbly Wake in a Ventilated Flow. Proceedings of the Tenth European Turbulence Conference, Barcelona, Spain, 2004.

Wu, T.Y., Whitney, A.K., Brennen, C. Cavity-flow wall effects and correction rules. Journal of Fluid Mechanics, vol. 49, part 2, pp. 223-256, printed in Great Britain, 1971.

http://cav.safl.umn.edu/

www.deepangel.com/html/the_squall/html

http://www.as.northropgrumman.com/products/ramics/index.html 


\section{APPENDIX A: Pressure Transducer Calibration Procedure}

The pressure transducers that measured the static pressure in the water tunnel test section, $\mathrm{P}_{\infty}$, the pressure inside the supercavity, $\mathrm{P}_{c}$, and the differential pressure, $\mathrm{P}_{0}-\mathrm{P}_{\infty}$, were calibrated every day before an experiment. The calibrations were performed using two mercury manometers, one for each transducer. Before calibration, the pressure lines were purged to ensure no air bubbles were trapped in them.

When calibrating the absolute pressure transducer that measured $\mathrm{P}_{\infty}$, one leg of the manometer was connected to the static pressure port while the other leg was connected to a tank filled with water whose level was at the same height as the static port in the water tunnel test section. The pressure in the tunnel was then varied by pulling a vacuum in the water tunnel. At each calibration point, the height of the mercury column and the pressure transducer output were recorded. Approximately 10 calibration points were taken each time the transducer was calibrated. After calibration, the pressure in the tunnel was relieved to atmospheric pressure.

After calibrating the test section absolute pressure transducer, the cavity pressure absolute pressure transducer was calibrated. To do this, the water tunnel was drained to a level that corresponded to half the height of the test section. The pressure tap in the back window of the test section, used for the test section pressure and differential pressure transducers, was underwater. It was ensured that the location for measuring pressure in the supercavity was in air and not influenced by the water in the test section. Care needed to be taken when this was done since the two are approximately at the same height. A vacuum was then pulled in the test section and the cavity pressure transducer was calibrated using the test section absolute pressure transducer. Opportunities

After calibrating the absolute pressure transducers, the differential pressure transducer was calibrated. When calibrating the differential pressure transducer, one leg of the manometer was connected to the stagnation pressure in the settling chamber of the test section while the other leg was connected to the static port in the test section. The differential pressure in the tunnel was then varied by changing the freestream velocity. 
Again, at each calibration point the height of the mercury column and the pressure transducer output were recorded.

After calibrating the three pressure transducers, the calibration curves were created by plotting the differential pressure given by the manometers versus transducer output. A straight line was then fit through the data for each transducer using a least squares fit.

The pressure transducer calibrations produced curves that were consistently linear, with R-squared values typically 0.9999 or higher for both the all transducers. Errors due to the least squares fit line were approximately $0.1 \mathrm{kPa}$ for both absolute pressure transducers. These errors lead to a maximum error in the measured velocity of $0.11 \mathrm{~m} / \mathrm{s}$, with typical errors being closer to $0.02 \mathrm{~m} / \mathrm{s}$. 


\section{APPENDIX B: Uncertainty Analysis \\ Sources of Error and Uncertainty}

Type A and Type B evaluations of uncertainty were conducted for the cavitation number. The error associated with taking the average of 3-5 readings for each data point during experimentation falls into Type A evaluation. This uncertainty is found according to

$$
\sigma_{a}=\frac{s}{\sqrt{n}}
$$

where $\mathrm{s}$ is the standard deviation of the readings and $\mathrm{n}$ is the number of readings. Type $\mathrm{B}$ evaluation of uncertainty accounts for uncertainty from any other information. Using calibration uncertainty and error propagation according to the root of the sum of the squares estimate (RSS), Type B evaluation of uncertainty is computed according to

$$
\sigma_{b}=\left[\sum_{i=1}^{n}\left(\sigma_{x_{i}} \frac{\partial R}{\partial x_{i}}\right)^{2}\right]^{1 / 2}
$$

where $\sigma_{\mathrm{b}}$ is the uncertainty in the quantity $\mathrm{R}$, which is of the form

$$
\mathrm{R}=f\left(\mathrm{x}_{1}, \mathrm{x}_{2}, \ldots, \mathrm{x}_{\mathrm{n}}\right) .
$$

The two types of uncertainty are then combined according to

$$
\sigma=\sqrt{\sigma_{a}^{2}+\sigma_{b}^{2}}
$$

For a cavitation number of 0.0882 , an uncertainty of 0.00083 was found, less than $1 \%$. This was a typical amount of error seen over the range tested. At very low Froude numbers, water was observed to occasionally splash onto the cavity pressure measurement location (similar to challenges observed by Schauer). When this was the case, an uncertainty up to $5 \%$ was not uncommon. Froude numbers where this was the case were not often tested because of this.

Given that the a variation in ventilation rate had little to no effect on cavitation number once a clear supercavity was established, error analysis was not computed for the entrainment coefficient since the focus of the experiments was the cavitation number. 
Also, the scales of the flowmeters used were not fine enough to determine the exact entrainment coefficient at the transition from re-entrant jet to twin-vortex type of closure. 


\section{APPENDIX C: Matlab code for supercavity analysis}

The following code was used to find the supercavity maximum diameter and half-length. The code opened a picture, taken during experimentation. The user would then select points along the top surface of the supercavity, beginning with the location of cavitator. This was then repeated along the bottom surface of the supercavity. A polynomial fit was then applied to the points that were selected. The curve generated for the top surface of the supercavity was then compared to the curve for the bottom surface. The maximum difference between the two was taken to be the maximum cavity diameter, with its location recorded as the half length. It was important for the first point chosen to be the cavitator as this is taken to be the location for the x-origin. For a given set of conditions, the average of 2-3 images was used as the result. As a reality check, Photoshop was used to see if the values produced by the code were realistic.

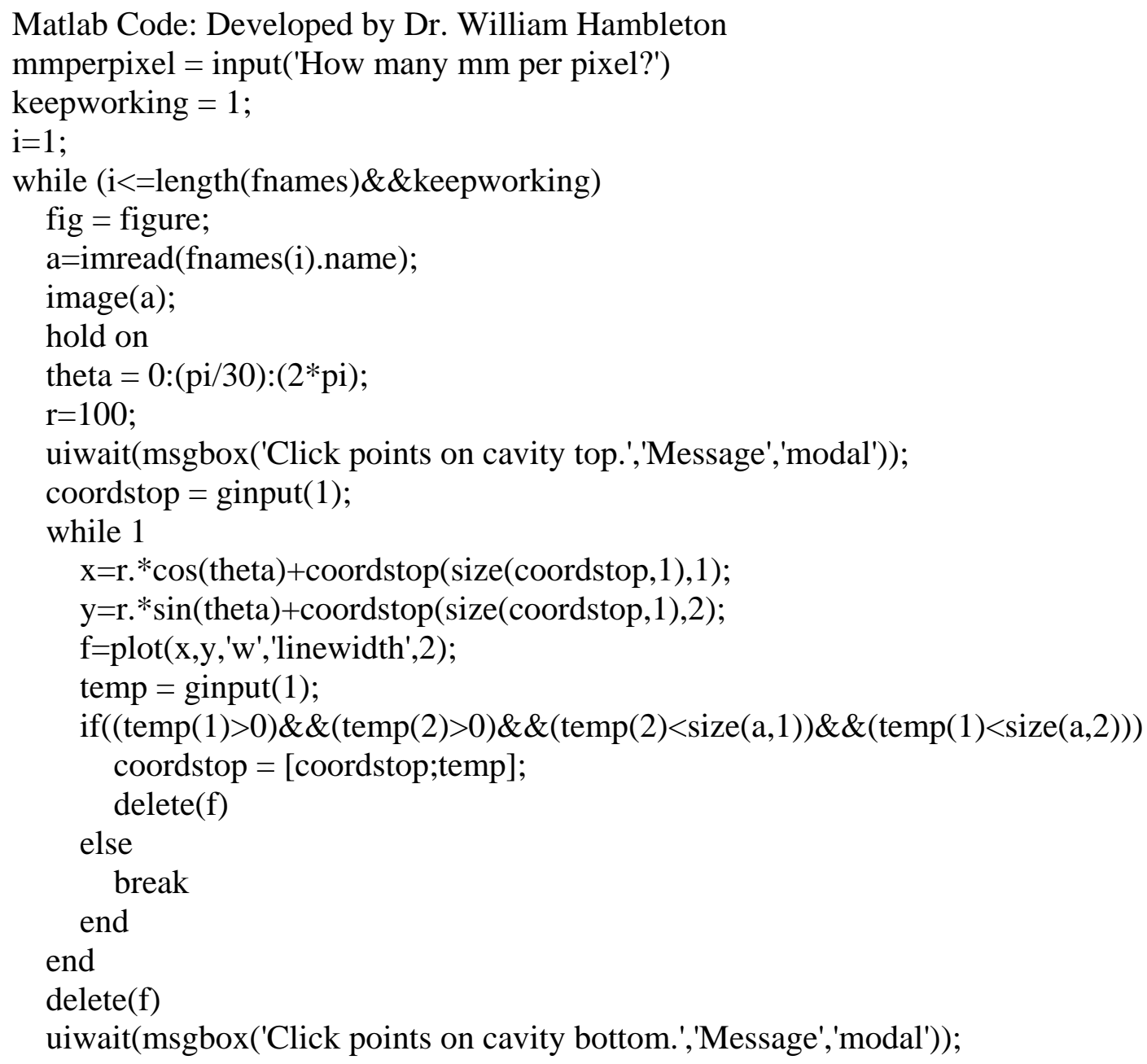




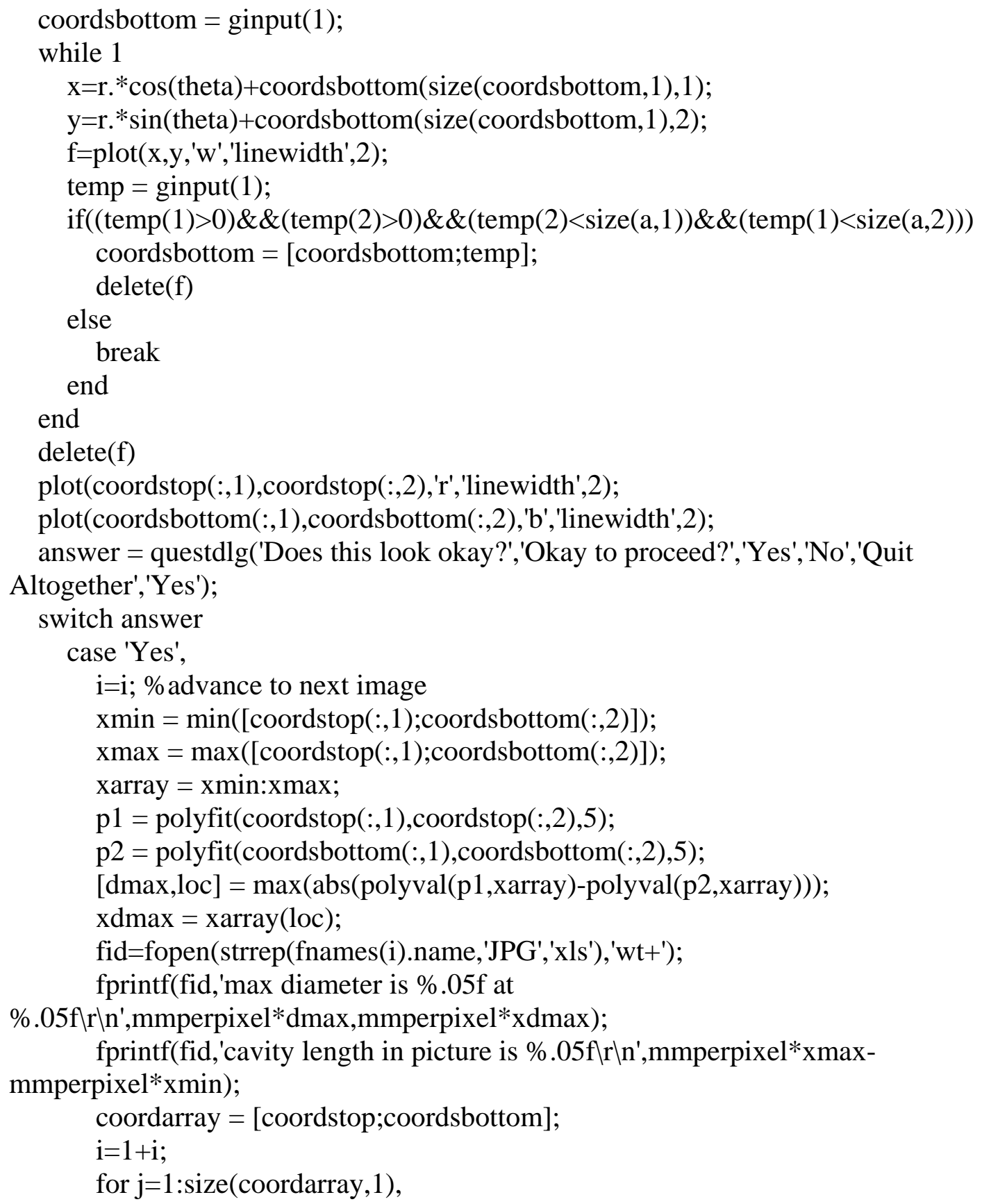

